Portland State University

PDXScholar

\title{
Theoretical and Experimental Validation of a CubeSat's L-Band Communication System
}

\author{
Evangelos Mastrogiannis \\ Portland State University
}

Follow this and additional works at: https://pdxscholar.library.pdx.edu/open_access_etds

Part of the Electrical and Computer Engineering Commons Let us know how access to this document benefits you.

Recommended Citation

Mastrogiannis, Evangelos, "Theoretical and Experimental Validation of a CubeSat's L-Band Communication System" (2020). Dissertations and Theses. Paper 5564.

https://doi.org/10.15760/etd.7438

This Thesis is brought to you for free and open access. It has been accepted for inclusion in Dissertations and Theses by an authorized administrator of PDXScholar. Please contact us if we can make this document more accessible: pdxscholar@pdx.edu. 
Theoretical and Experimental Validation of a CubeSat's L-Band Communication System

by

Evangelos Mastrogiannis

A thesis submitted in partial fulfillment of the requirements for the degree of

\author{
Master of Science \\ in \\ Electrical and Computer Engineering
}

Thesis Committee:

Richard Campbell, Chair

Branimir Pejčinović

Andrew Greenberg

Portland State University

2020 
(C) 2020 Evangelos Mastrogiannis 


\begin{abstract}
An L-band uplink communication system was designed and validated in the lab for a CubeSat satellite operating in low Earth orbit (LEO). This paper investigates communication link analysis, discusses the design strategy for an inexpensive CubeSat receiver operating in L-band with a moderate power Earth station transmitter, and validates the link budget with prototype hardware using an anechoic chamber.

A receiver's required carrier-to-noise ratio (CNR) establishes the basis for a link budget. The requirement for a digital communication link is set by the bit-error-rate (BER) requirement of a chosen communication protocol which is inversely related to the energy per bit to noise power spectral density $\left(\mathrm{E}_{\mathrm{b}} / \mathrm{N}_{0}\right)$ of a chosen modulation scheme. The carrier power level can be controlled; however, noise power and specifically thermal noise power can only partially be controlled. Through investigation of thermal noise power effects on receiver front-end hardware, a low power and low system noise temperature receiver was designed utilizing a downconverter with inexpensive commercial off-the-shelf (COTS) components. In addition, the link analysis minimized Earth station transmit power; however, for the purpose of this thesis a simple low power transmitter was designed.

Preliminary measurements of the designed receiver and transmitter were taken to evaluate performance. Measured system noise temperature of the receiver was used for link analysis which compared to calculations. For link budget validation, closed system testing with injected noise power was conducted for a validation baseline before testing in an anechoic chamber which allowed for antenna testing in a controlled
\end{abstract}


thermal noise environment. A Y-factor with correction measurement method was used with a spectrum analyzer to precisely set expected carrier and noise power levels at the receiver's front-end. The same method was used to verify the integrity of the anechoic chamber by measuring the receiver's antenna noise temperature. Measurement results compared closely to theoretical BER vs. $E_{b} / N_{0}$ plots after a revised CNR to $E_{b} / N_{0}$ relationship was conceived for the binary frequency-shift keying (BFSK) modulation schemes used. In addition, a small and expected modulation implementation loss was shown, and performance limitations of the sub-gigahertz transceiver IC were discovered. 
For my wife, Julie, and my kids, Phoebe and Gabriel. 


\section{Acknowledgments}

I would like to express my deep gratitude to my advisor Dr. Richard Campbell for his continual encouragement, inspiration, and support over the years of my graduate program. In addition, I appreciate his patience and faith in completing my thesis. I would like to thank Dr. Branimir Pejčinović for his willingness to participate as one of my committee members and providing in-depth editing advice of the thesis document. In addition, I am grateful for him allowing me access to the Integrated Circuit Design and Test lab.

I would also like to thank Mr. Andrew Greenberg for his suggestion and encouragement to write a thesis on the research and development I was already working on in the Portland State Aerospace Society. In addition, for his willingness to participate as one of my committee members.

I am particularity grateful for Mr. Glenn LeBrasseur for providing unbiased mentorship through my research and development of this project. In addition, allowing me access to the RF Lab which provided access to an anechoic chamber.

I would like to offer my special thanks to Mr. Malay Das for his commitment to helping solve embedded firmware challenges for this project, and I would like to extend my thanks to the staff at the Electronics Prototyping Lab for their assistance with equipment.

I would like to express my appreciation to my speech therapist Mrs. Nicole Jonak for her assistance and encouragement during my thesis defense preparation, and to my dear friend Mr. Tyler Blomdahl for offering his proofreading advice and inspiring me to 
be the best version of myself. Finally I wish to thank my family for their support and encouragement over the course of my graduate program and thesis. 


\section{Table of Contents}

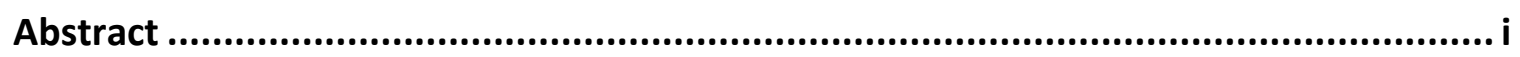

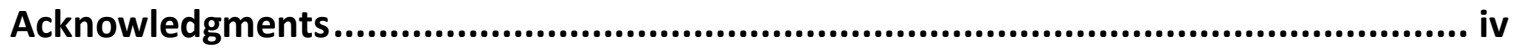

List of Tables .......................................................................................... ix

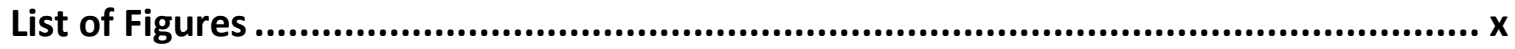

List of Abbreviations..................................................................................

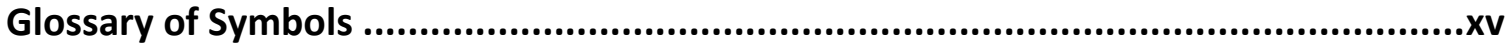

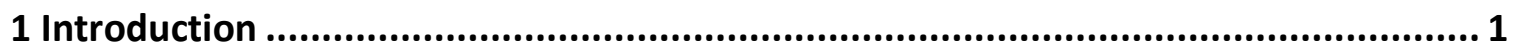

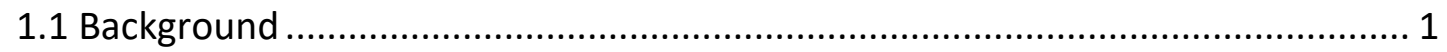

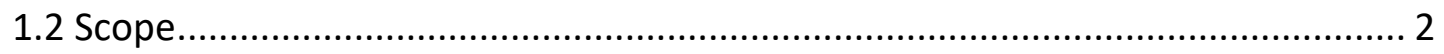

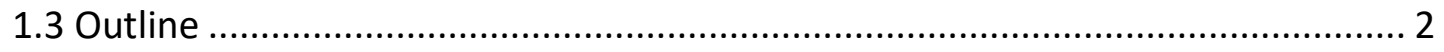

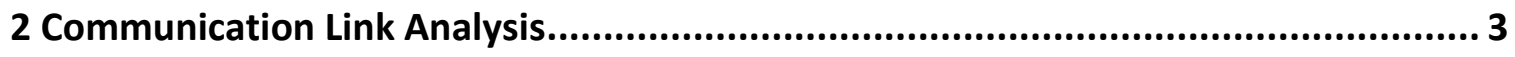

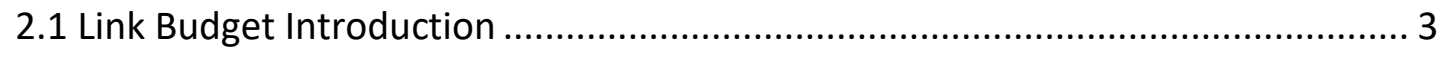

2.2 Thermal Noise ............................................................................................. 5

2.2.1 Blackbody Radiation ........................................................................ 5

2.2.2 Johnson-Nyquist Noise ................................................................... 7

2.2.3 Antenna Noise Temperature .......................................................... 8

2.3 Effective System Noise Temperature ..................................................... 9

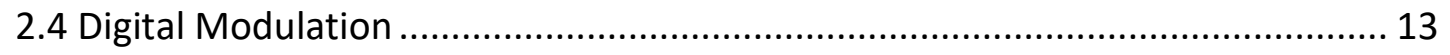

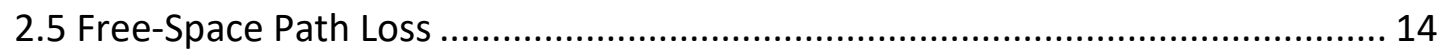

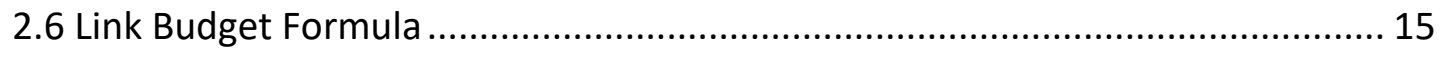

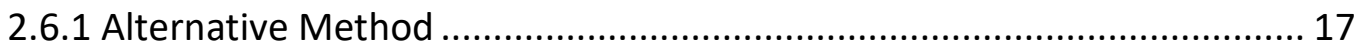

2.6.2 Receiver Figure of Merit ................................................................. 18 


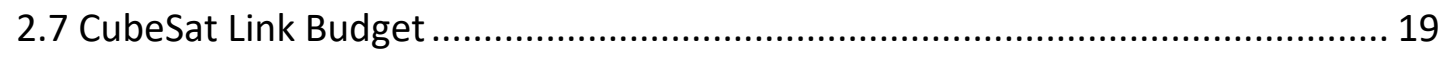

2.7.1 Minimum Receiver Power ................................................................... 20

2.7.2 Minimum Transmitter Power ................................................................. 22

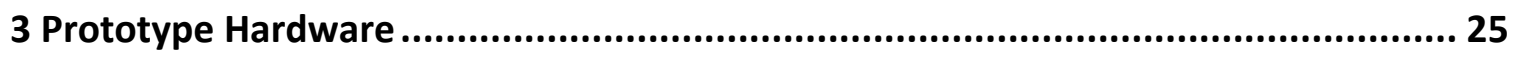

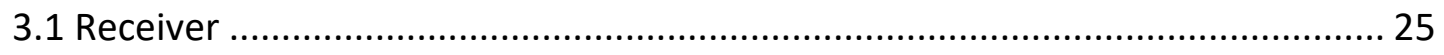

3.1.1 Superhet Architecture .................................................................... 26

3.1.2 CubeSat Receiver Design .................................................................. 28

3.1.3 CubeSat Receiver Construction .............................................................. 31

3.1.4 First Stage LNA Design ........................................................................... 33

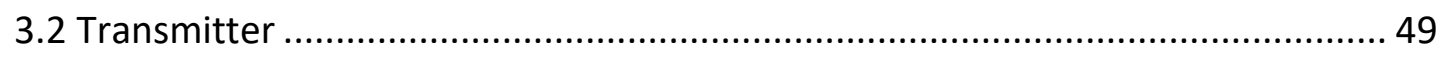

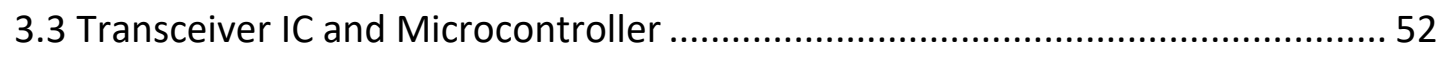

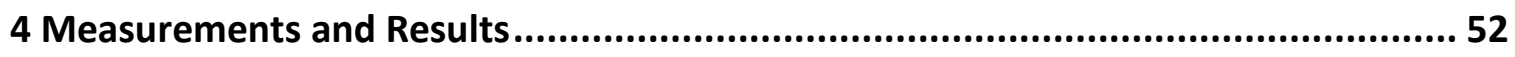

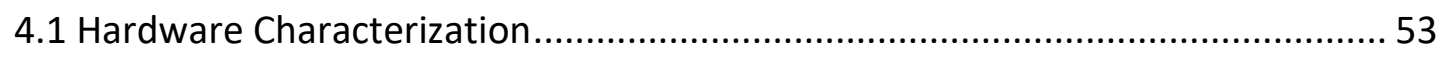

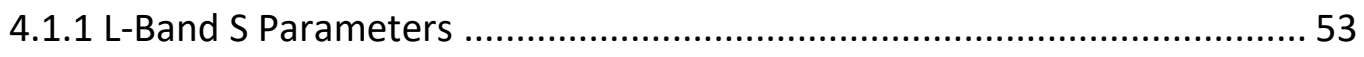

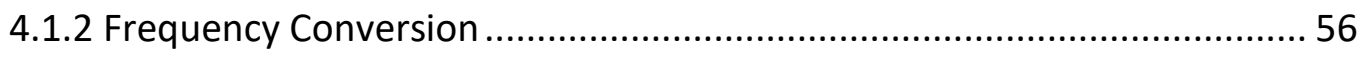

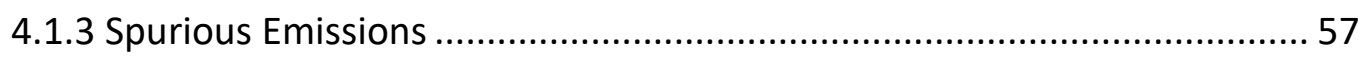

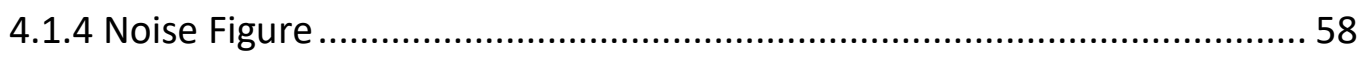

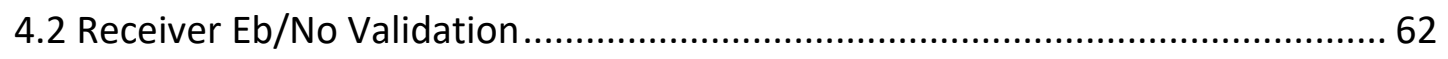

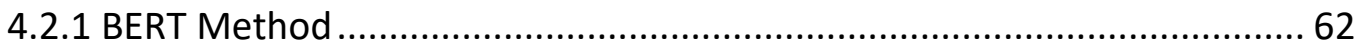

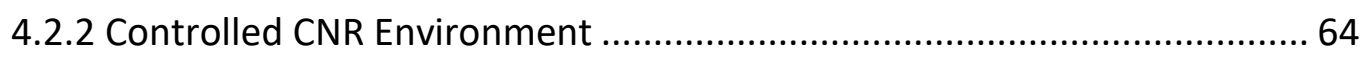

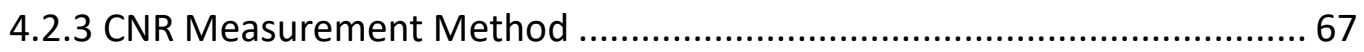

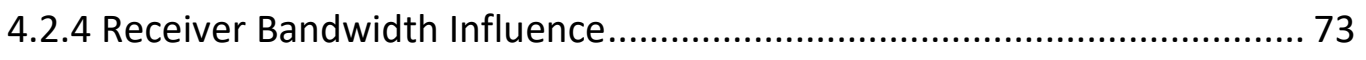

4.2.5 Final Validation Formulas ....................................................................... 79

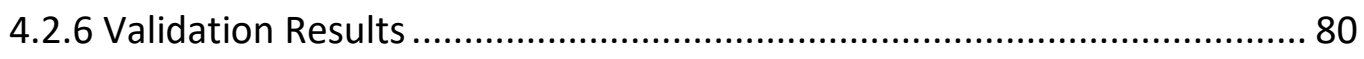

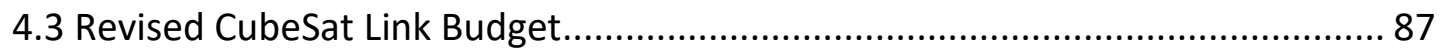

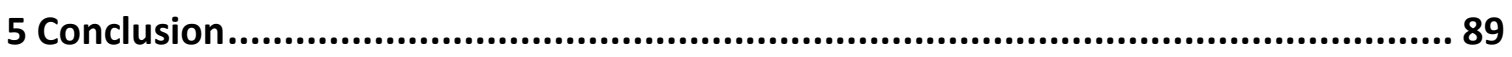




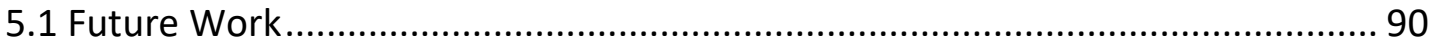

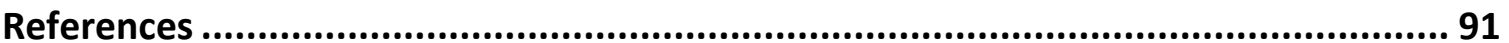




\section{List of Tables}

Table 2.1: Parameter summary of effective system noise temperature ......................................2 21

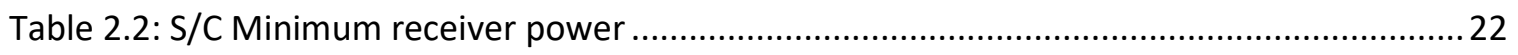

Table 2.3: FSPL from various elevations for $400 \mathrm{~km} \mathrm{S/C} \mathrm{altitude} \mathrm{.............................................23}$

Table 2.4: ES minimum Effective Isotropic Radiated Power (EIRP) ..........................................24

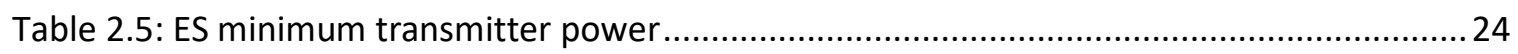

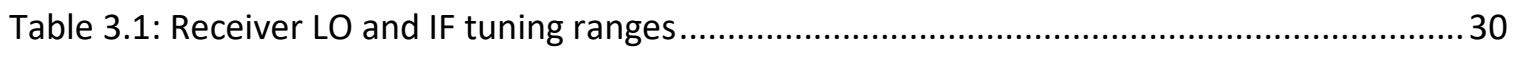

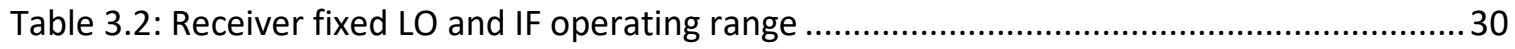

Table 4.1: L-band receiver input port spurious emissions ....................................................... 58

Table 4.2: Noise temperature of front-end components of L-band receiver evaluated with Friis formula (prototype box used for validation) .......................................................6 60

Table 4.3: Noise temperature of L-band receiver excluding antenna feed network evaluated with Friis formula (CubeSat receiver used for link budgets) ...............................61

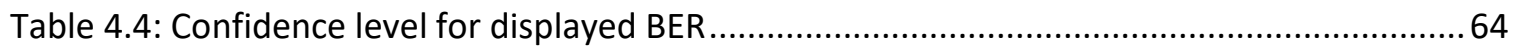

Table 4.5: Summary of Y-factor with correction method parameters .........................................69

Table 4.6: Summary of receiver bandwidth to deviation for all modulation schemes .................84

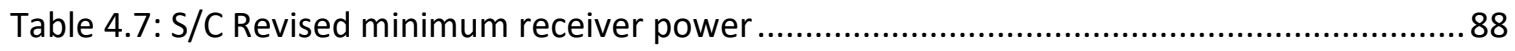

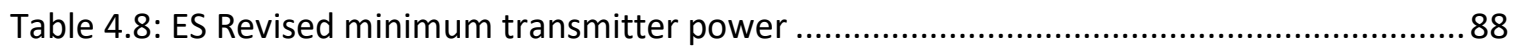




\section{List of Figures}

Figure 2.1: Planck's blackbody radiation curves, Spectral Radiance vs. Wavelength..................... 6

Figure 2.2: Planck's blackbody radiation curves of common sources ..........................................6

Figure 2.3: Spectral Radiance for Planck's Law compared to Rayleigh-Jeans Law ........................ 7

Figure 2.4: Effective System Noise Temperature Block Diagram ............................................ 12

Figure 2.5: BER vs. $\mathrm{E}_{\mathrm{b}} / \mathrm{N}_{0}$ for BPSK, MSK, and BFSK modulation schemes ..................................14

Figure 2.6: Alternative Effective System Noise Temperature Block Diagram...............................17

Figure 3.1: Minimal design superheterodyne block diagram ...................................................27

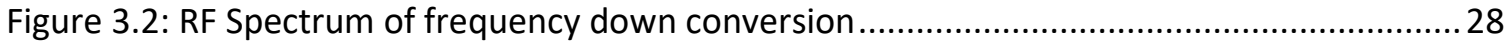

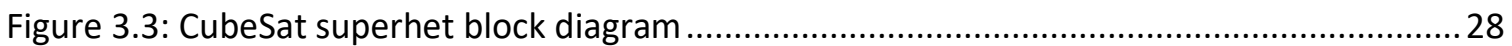

Figure 3.4: Detailed CubeSat receiver block diagram ...............................................................29

Figure 3.5: L-band receiver evaluation boards designed and assembled by author .....................32

Figure 3.6: Prototype L-band receiver (purple boards) in steel box. Designed and assembled by author

Figure 3.7: First stage L-band LNA with Infineon Technologies BFP740F RF BJT. Designed

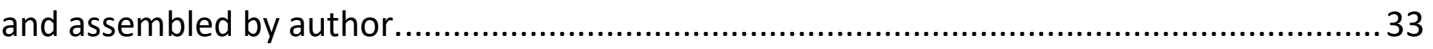

Figure 3.8: ADS schematic for initial device characterization of Infineon BFP740F RF BJT ...........36

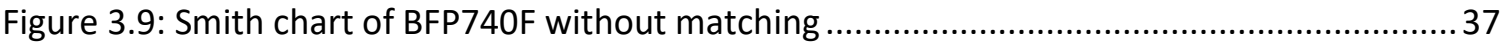

Figure 3.10: S parameter magnitude plot of BFP740F without matching …...............................37

Figure 3.11: Smith charts of optimal source/load, constant gain circles, and stability circles evaluated at $1265 \mathrm{MHz}$ for BFP740F w/o matching. Source (left, red) and Load (right, blue).. .38

Figure 3.12: ADS schematic for stability improved initial device of Infineon BFP740F RF BJT with stability improvements added. 39

Figure 3.13: S parameter magnitude plot of BFP740F with stability improvements added. ........40

Figure 3.14: Smith charts of optimal source/load, constant gain circles, and stability circles evaluated at $1265 \mathrm{MHz}$ for BFP740F with stability improvements added. Source (left, red) and Load (right, blue).

Figure 3.15: Stability Factor, K, plot of initial BFP740F design before(left) and after (right) the addition of the stability improvements. 
Figure 3.16: Smith chart of optimal source impedance with constant gain circles, noise figure circle, and stability circle evaluated at $1265 \mathrm{MHz}$ for initial BFP740F conditionally stable design.

Figure 3.17: Smith charts of source impedance matching network. Series inductor only (left), and complete matching network with biasing components (right).

Figure 3.18: ADS schematic of stability improved BFP740F with source matching network added, and configured for source impedance analysis. .43

Figure 3.19: Smith chart of BFP740F with input matching network and stability improvements (left). Smith chart of optimal load impedance, constant gain circles, and stability circle evaluated at $1265 \mathrm{MHz}$ (right).

Figure 3.20: S parameter magnitude plot of BFP740F with input matching network and stability improvements.

Figure 3.21: ADS schematic of stability improved BFP740F with source and load matching network added, and configured for load impedance analysis.

Figure 3.22: Smith charts of load impedance matching network. Shunt inductor only (left), and complete matching network with biasing components (right). .46

Figure 3.23: ADS schematic of BFP740F showing updated placement of output shunt stability resistor.

Figure 3.24: Final L-band LNA design with Infineon BFP740F RF BJT .47

Figure 3.25: Smith chart of BFP740F final design (left) with stability factor plot (right) ..............48

Figure 3.26: S parameter magnitude plot of BFP740F BJT final design ....................................... 48

Figure 3.27: Final EAGLE CAD schematic of L-band LNA with Infineon BFP740F RF BJT ............... 49

Figure 3.28: L-band Prototype block diagram ……................................................................. 50

Figure 3.29: RF Spectrum of frequency up conversion .............................................................. 50

Figure 3.30: L-band (1265 MHz) hairpin filter designed by author ..........................................51

Figure 3.31: Prototype L-band transmitter (purple boards) in steel box. Designed and assembled by author.

Figure 4.1: Smith chart (left) and stability factor (right) of measured BFP740F L-band LNA prototype .53

Figure 4.2: Magnitude plot of the measured BFP740F L-band LNA prototype ............................54

Figure 4.3: L-band receiver RF portion front-end components in VNA measurement ..................55 
Figure 4.4: S parameter magnitude plot (left) and stability factor (right) of measured L-

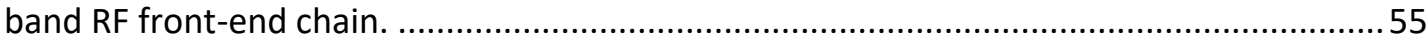

Figure 4.5: L-band receiver front-end in a conversion gain measurement ................................57

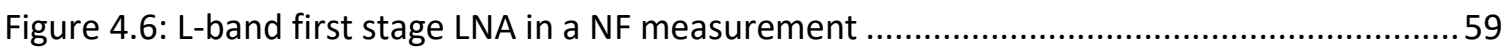

Figure 4.7: L-band prototype receiver front-end in a NF measurement ...................................60

Figure 4.8: L-band receiver showing active DVK-2 main board with BER display ........................63

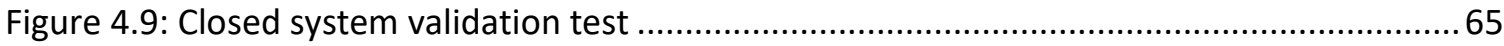

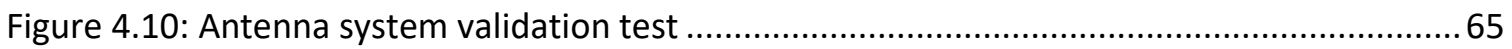

Figure 4.11: Antenna system validation test with terrestrial noise..........................................66

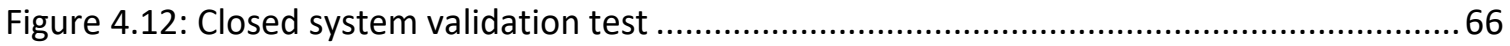

Figure 4.13: Antenna system validation test in anechoic chamber ...........................................67

Figure 4.14: Lab equipment showing received signal on spectrum analyzer .............................67

Figure 4.15: RF Spectrum of very weak carrier (left), receiver noise temp. contribution

(mid), and resulting corrected carrier power with effective system noise power

(right)

Figure 4.16: Measured very weak 60 kbps MSK with 3.14 dB CNR on SA via Y-factor correction method

Figure 4.17: Measured 60 kbps MSK with $16.7 \mathrm{~dB}$ CNR on SA with Y-factor correction method 72

Figure 4.18: AX5043 transceiver IC radio parameters shown in AX-RadioLAB software application .74

Figure 4.19: BER vs. $\mathrm{E}_{\mathrm{b}} / \mathrm{N}_{0}$ plot for 60 kbps MSK using data rate method ................................... 75

Figure 4.20: $\mathrm{BER}$ vs. $\mathrm{E}_{\mathrm{b}} / \mathrm{N}_{0}$ plot for $60 \mathrm{kbps} \mathrm{MSK}$ using receiver bandwidth method ....................76

Figure 4.21: Modulation implementation loss for receiver bandwidth method.........................77

Figure 4.22: $B E R$ vs. $E_{b} / N_{0}$ plot for 60 kbps $M S K$ using revised $C N R$ to $E_{b} / N_{0}$ relationship...........78

Figure 4.23: Modulation implementation loss for revised method ............................................. 78

Figure 4.24: $\mathrm{BER}$ vs. $\mathrm{E}_{\mathrm{b}} / \mathrm{N}_{0}$ plot for $9.6 \mathrm{kbps} \mathrm{BFSK}, \mathrm{h}=0.625$ for various test methods ................. 81

Figure 4.25: $\mathrm{BER}$ vs. $\mathrm{E}_{\mathrm{b}} / \mathrm{N}_{0}$ plot for $60 \mathrm{kbps} \mathrm{MSK}$ for various test methods ................................ 82

Figure 4.26: $\mathrm{BER}$ vs. $\mathrm{E}_{\mathrm{b}} / \mathrm{N}_{0}$ plot for $120 \mathrm{kbps} \mathrm{MSK}$ for various test methods .............................83

Figure 4.27: BER vs. $E_{b} / N_{0}$ plot for 60 kbps BFSK, $h=0.75$ for various test methods ...................... 84

Figure 4.28: Modulation index influence on BER vs. $\mathrm{E}_{\mathrm{b}} / \mathrm{N}_{0}$ plot for $60 \mathrm{kbps}$............................... 86 
Figure 4.29: Modulation index influence on Implementation Loss vs. BER plot for $60 \mathrm{kbps}$........86 


\section{List of Abbreviations}

\begin{tabular}{|c|c|}
\hline ADC & Analog to Digital Converter \\
\hline BER & Bit-Error-Rate \\
\hline BERT & Bit-Error-Rate Test \\
\hline BFSK & Binary Frequency-Shift Keying \\
\hline BJT & Bipolar Junction Transistor \\
\hline BPF & Band-Pass Filter \\
\hline$C L$ & Confidence Level \\
\hline CNR & Carrier-to-Noise Ratio \\
\hline COTS & Commercial Off-The-Shelf \\
\hline$d B$ & Decibels of relative power \\
\hline $\mathrm{dBc}$ & Decibels relative to the carrier \\
\hline$d B i$ & Decibels relative to an isotropic antenna \\
\hline $\mathrm{dBm}$ & Decibel-milliwatt of absolute power \\
\hline DSP & Digital Signal Processing \\
\hline DUT & Device Under Test \\
\hline EMI & Electromagnetic Interference \\
\hline ES & Earth Station \\
\hline FIFO & First-In, First-Out \\
\hline FSPL & Free-Space Path Loss \\
\hline IARU & International Amateur Radio Union \\
\hline IF & Intermediate Frequency \\
\hline ITU & International Telecommunication Union \\
\hline L-band & IEEE frequency band, $1 \mathrm{GHz}$ to $2 \mathrm{GHz}$ \\
\hline LEO & Low Earth Orbit \\
\hline LNA & Low Noise Amplifier \\
\hline LO & Local Oscillator \\
\hline LPF & Low-Pass Filter \\
\hline $\mathrm{OP} 1 \mathrm{~dB}$ & Output $1 \mathrm{~dB}$ compression point \\
\hline PCB & Printed Circuit Board \\
\hline PN & Pseudorandom Number \\
\hline$S / C$ & Spacecraft \\
\hline SAW & Surface Acoustic Wave \\
\hline SNR & Signal-to-Noise Ratio \\
\hline TCXO & Temperature Controlled Crystal Oscillator \\
\hline TDR & Time-Domain Reflectometry \\
\hline
\end{tabular}




\section{Glossary of Symbols}

\begin{tabular}{|c|c|}
\hline$A_{e}$ & Effective antenna aperture \\
\hline$B$ & Bandwidth \\
\hline$B_{N}$ & Channel bandwidth \\
\hline$B_{R}$ & Receiver bandwidth \\
\hline$c$ & Speed of light in a vacuum \\
\hline$C$ & Received carrier power \\
\hline$C_{0}$ & Carrier power spectral density \\
\hline$C / N$ & Carrier-to-noise ratio \\
\hline$d$ & Slant range \\
\hline$E_{b}$ & Energy per bit \\
\hline$E_{b} / N_{0}$ & Energy per bit to noise power spectral density \\
\hline$f$ & Frequency \\
\hline$F$ & Noise factor \\
\hline$G$ & Gain of an active device $\left(P_{\text {out }} / P_{\text {in }}\right)$ \\
\hline$G_{\text {ant }}$ & Antenna gain \\
\hline$h$ & Planck's constant \\
\hline$h_{m}$ & Modulation index \\
\hline$H$ & Spacecraft altitude \\
\hline$k$ & Boltzmann's constant \\
\hline$L$ & Loss of a passive device $\left(\mathrm{P}_{\text {in }} / \mathrm{P}_{\text {out }}\right)$ \\
\hline$L_{\text {Atmos }}$ & Atmospheric and scintillation losses \\
\hline$L_{\text {Feed }}$ & Antenna feed line losses \\
\hline$L_{F S P L}$ & Free-space path loss \\
\hline$L_{M o d}$ & Modulation implementation loss \\
\hline$L_{\text {Point }}$ & Antenna pointing losses \\
\hline$L_{\lambda}$ & Spectral Radiance per unit wavelength \\
\hline$M_{L}$ & Link margin \\
\hline$N$ & Noise power \\
\hline$N_{0}$ & Noise power spectral density \\
\hline$N_{S}$ & Effective system noise power \\
\hline$N F$ & Noise figure \\
\hline$P_{A}$ & Antenna noise power \\
\hline$P_{N}$ & Noise power \\
\hline$P_{R_{x}}$ & Received carrier power \\
\hline$P_{T_{x}}$ & Transmitted carrier power \\
\hline
\end{tabular}




$\begin{array}{ll}Q & \text { Q-function } \\ R_{b} & \text { Data bitrate } \\ R_{E} & \text { Earth's radius } \\ T & \text { Temperature } \\ T_{0} & \text { Reference temperature, typically } 290 \text { Kelvin } \\ T_{A} & \text { Antenna noise temperature } \\ T_{e} & \text { Effective temperature } \\ T_{R} & \text { Receiver noise temperature } \\ T_{S} & \text { Effective system noise temperature } \\ \theta & \text { Spacecraft elevation } \\ \lambda & \text { Wavelength }\end{array}$




\section{Introduction}

A communication uplink is a wireless RF communication system with data information transmitted from an Earth station (ES) and received by a spacecraft (S/C). In order to validate a satellite uplink, an active satellite's uplink must be analyzed, or an imitation of a system must be analyzed in a similar RF noise environment. The latter option was chosen.

In this thesis the hardware for a low power satellite receiver was designed using inexpensive commercial off-the-shelf (COTS) components which would operate successfully with moderate transmitted power from an Earth station. The L-band receiver was designed to operate in low Earth orbit (LEO) at $1.265 \mathrm{GHz}$ while establishing a $120 \mathrm{kbps}$ communication link at a $10^{\circ}$ minimum spacecraft elevation. The receiver and a test transmitter were constructed, and the communication link was validated in the lab by creating a communication channel with controlled RF noise.

\subsection{Background}

Motivation for this project originated from the primary communication system uplink goal for the Amateur CubeSat satellite OreSat1 being developed by the Portland State Aerospace Society (PSAS) [1]. There have not been many Amateur radio satellites utilizing the L-band $(23 \mathrm{~cm})$ Amateur satellite radio band for an uplink communication channel compared to VHF ( $2 \mathrm{~m}$ band) and UHF (70 $\mathrm{cm}$ band) [2] [3]. Less bandwidth utilized by the community implies less interference from spacecraft and Earth stations. 
In addition, the $23 \mathrm{~cm}$ satellite uplink band is wider than the others allowing for wider bandwidths and hence higher data rates.

\subsection{Scope}

An imitation L-band uplink communication system was constructed in the lab with the use of an anechoic chamber. The primary challenge of any communication system is validating the threshold of the receiver. The threshold of a receiver is how well it operates at a minimum received signal level. More specifically, the threshold of a digital receiver is the balance of the minimum required received energy per bit, the data rate, and the total received RF noise power for a specific bandwidth. Using an anechoic chamber, the received RF noise power could be controlled. The received energy per bit and RF noise power could then be measured to validate the threshold of the receiver. With the threshold of the L-band receiver known, the specification can then be used for estimating the uplink communication system of the CubeSat. This is referred to as the system's link budget and specifically the uplink budget.

\subsection{Outline}

This paper is organized in three major chapters: theory, hardware, and measurements and results. A brief description of the following three chapters is given below.

\section{Chapter 2: Communication Link Analysis}

Derivation of a communication system link budget with thermal noise theory and initial analysis of the CubeSat's proposed L-band uplink budget. 


\section{Chapter 3: Prototype Hardware}

Theory and design of the CubeSat's L-band receiver used for experimental validation including the first stage LNA design with a brief discussion of the transmitter designed.

\section{Chapter 4: Measurements and Results}

Hardware characterization measurements, the L-band receiver threshold validation experiment and results, and the revised analysis of the CubeSat's proposed L-band uplink budget.

\section{Communication Link Analysis}

A link budget analyzes the performance of a communication system. For a wireless digital communication system, a link budget estimates if a system will operate successfully within an error rate constraint. The cause of errors in a system are from various types of RF noise and possible RF interference. This chapter provides theoretical background on communication link analysis including the link budget design for this thesis.

\subsection{Link Budget Introduction}

A link budget consists of two major factors, the carrier power budget and the effects of RF noise power and interference on that power budget. The combination of these is referred to as the link budget which evaluates the receiver's carrier-to-noise 
ratio (CNR) and compares it to the required CNR of the receiver for a specific error-rate. The carrier power budget is straightforward, a carrier with a modulated signal is transmitted with a known power level $\left(P_{T_{x}}\right)$ and the received carrier power level $\left(P_{R_{x}}\right)$ is calculated by summing all the gains and losses along the communication channel. The communication channel includes antenna gains $\left(G_{A n t}\right)$, antenna pointing losses including S/C orientation $\left(L_{\text {Point }}\right)$, antenna feed losses $\left(L_{\text {Feed }}\right)$, free-space path loss (FSPL) $\left(L_{F S P L}\right)$, and atmospheric losses including scintillation $\left(L_{\text {Atmos }}\right)[4]$. An antenna's gain is defined at the ratio of the maximum produced power from the antenna's directivity and efficiency $\left(P_{\text {Ant.Max }}\right)$ relative to an isotropic antenna $\left(P_{\text {Ant.Isotropic }}\right)$ shown in Equation (2.1) [5]. A loss $(L)$ is defined as the ratio of input power $\left(P_{\text {in }}\right)$ to output power $\left(P_{\text {out }}\right)$ shown in Equation (2.2). The receiver's carrier power budget is shown in Equation (2.3).

$$
\begin{gathered}
G_{\text {Ant }}=\frac{P_{\text {Ant.Max }}}{P_{\text {Ant.Isotropic }}} \\
L=\frac{P_{\text {in }}}{P_{\text {out }}} \\
P_{R_{x}}=\frac{P_{T_{x}} G_{T_{x} A n t} G_{R_{x} A n t}}{L_{\text {Point }} L_{T_{x} . F e e d} L_{F S P L} L_{\text {Atmos }}}
\end{gathered}
$$

The effects of RF noise power and interference on the channel is a complicated subject which varies for each application. RF interference on the channel from outside sources including other spacecraft or Earth stations are assumed to be negligible for this study. The source and effects of internal RF interferences, created by the receiver itself, are discussed and some sources analyzed and measured. The primary contribution of noise power on most communication channels is from RF thermal noise power, from the 
environment and within the receiver itself. Equation (2.4) shows how received carrier power $(C)$ is related to the effective system noise power $\left(N_{S}\right)$ which includes received noise power from the environment and the receiver's internally generated noise power.

$$
\frac{P_{R_{x}}}{P_{\text {Eff.Sys.Noise }}}=\frac{C}{N_{S}} \geq C N R_{\text {Required }}
$$

\subsection{Thermal Noise}

Before a link budget is discussed further, thermal noise sources and antenna noise temperature are reviewed.

\subsubsection{Blackbody Radiation}

All objects above absolute zero temperature radiate and absorb electromagnetic energy. A perfect absorber is called a blackbody, and therefore a perfect radiator is called a blackbody radiator. The brightness or radiated electromagnetic spectral power per unit steradian referred to as spectral radiance $\left(L_{\lambda}\right)$ per unit wavelength $(\lambda)$ of a perfect blackbody is only a function of its temperature $(T)$. This relationship is given by Planck's Law shown in Equation (2.5) with units of $\mathrm{Wm}^{-2} \mathrm{sr}^{-1}$ per unit wavelength or typically $\mathrm{Wm}^{-2} \mathrm{~m}^{-1} \mathrm{sr}^{-1}$ [4] [6]. With dependence on Planck's constant $(h)$ and the speed of light in a vacuum $(c)$. Figure 2.1 below shows Planck's blackbody radiation curves, spectral radiance vs wavelength.

$$
L_{\lambda}=\frac{2 h c^{2}}{\lambda^{5}} \frac{1}{e^{h c / k T \lambda}-1}
$$




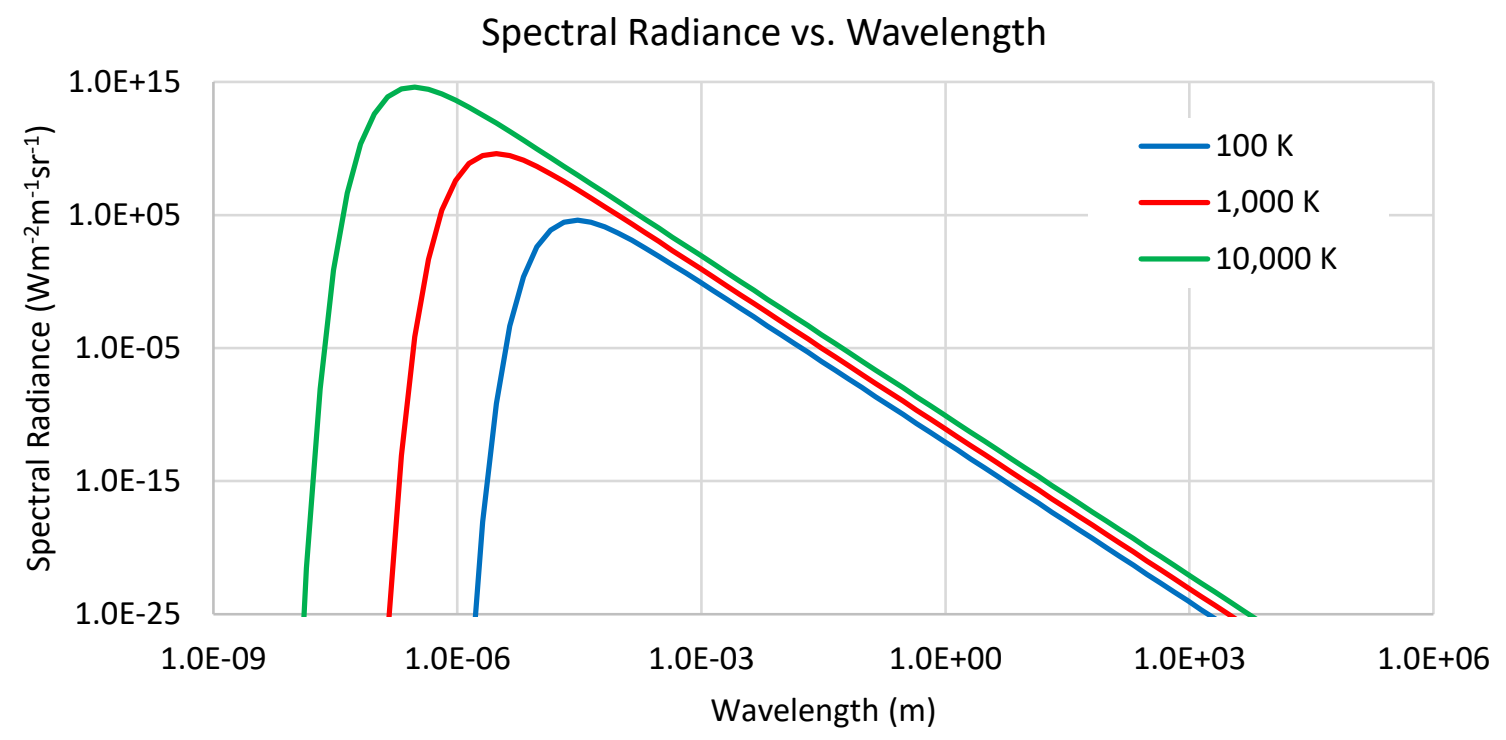

Figure 2.1: Planck's blackbody radiation curves, Spectral Radiance vs. Wavelength

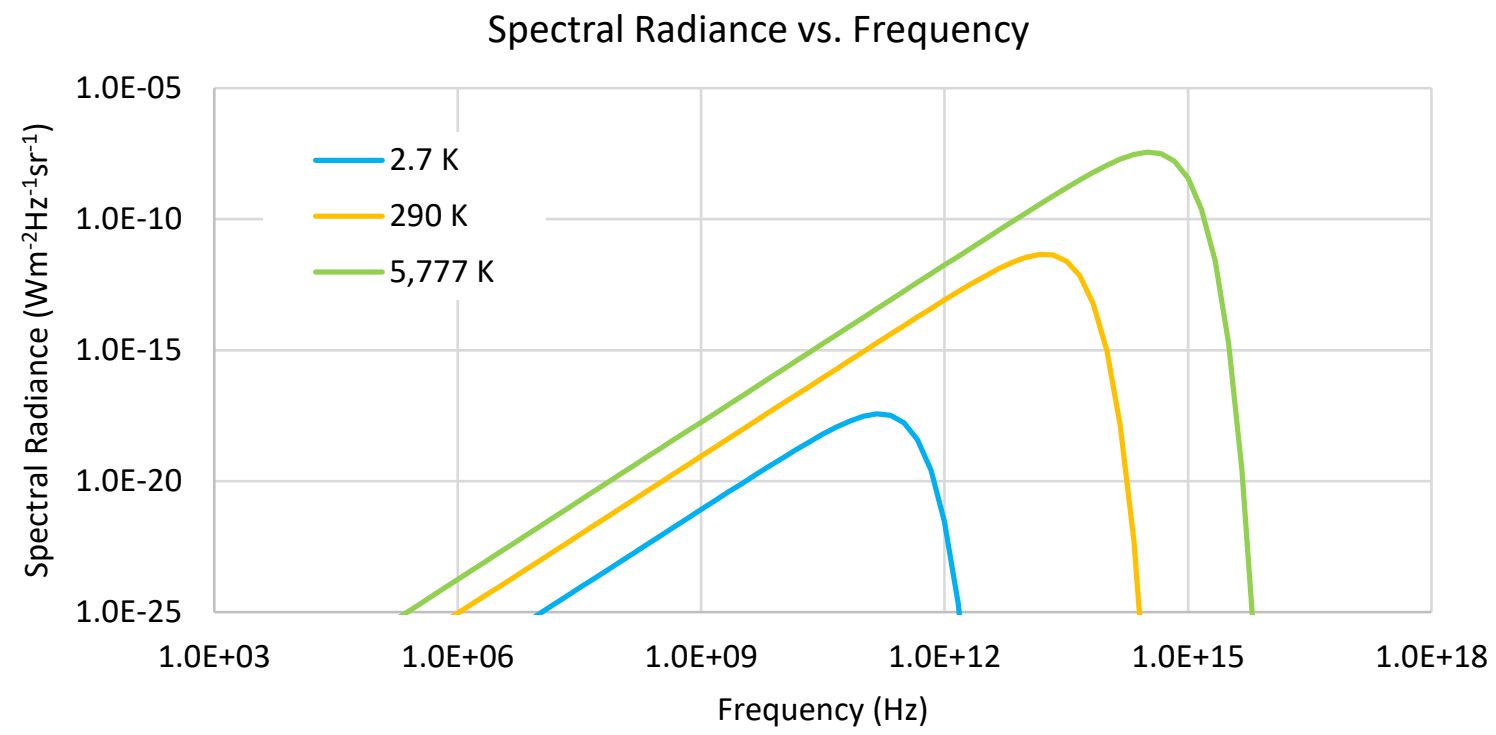

Figure 2.2: Planck's blackbody radiation curves of common sources

Thermal noise power radiated from the environment can be approximated by blackbody radiation. The sun, Earth, and cosmic microwave background are approximate blackbody radiators with an effective temperature of 5,777, 290, and 2.7 Kelvin respectively shown above in Figure 2.2 of spectral radiance per unit frequency, with units of $\mathrm{Wm}^{-2} \mathrm{~Hz}^{-1} \mathrm{sr}^{-1}$. Note at extremely low temperatures similar to the cosmic 
microwave background the power spectral density peaks at around $200 \mathrm{GHz}$ which is close to the upper range in the microwave spectrum, and at $290 \mathrm{~K}$ it peaks at around 20 THz. In conclusion, blackbody radiation affects all microwave communication systems. In addition, Planck's brightness formula is simplified by the Rayleigh-Jeans Law which is shown in Equation (2.6) [6]. A comparison of Planck's Law to Rayleigh-Jeans approximation for Spectral Radiance is shown in Figure 2.3.

$$
L_{\text {Rayleigh-Jeans }}=\frac{2 k T}{\lambda^{2}}=\frac{2 k T f^{2}}{c^{2}}
$$

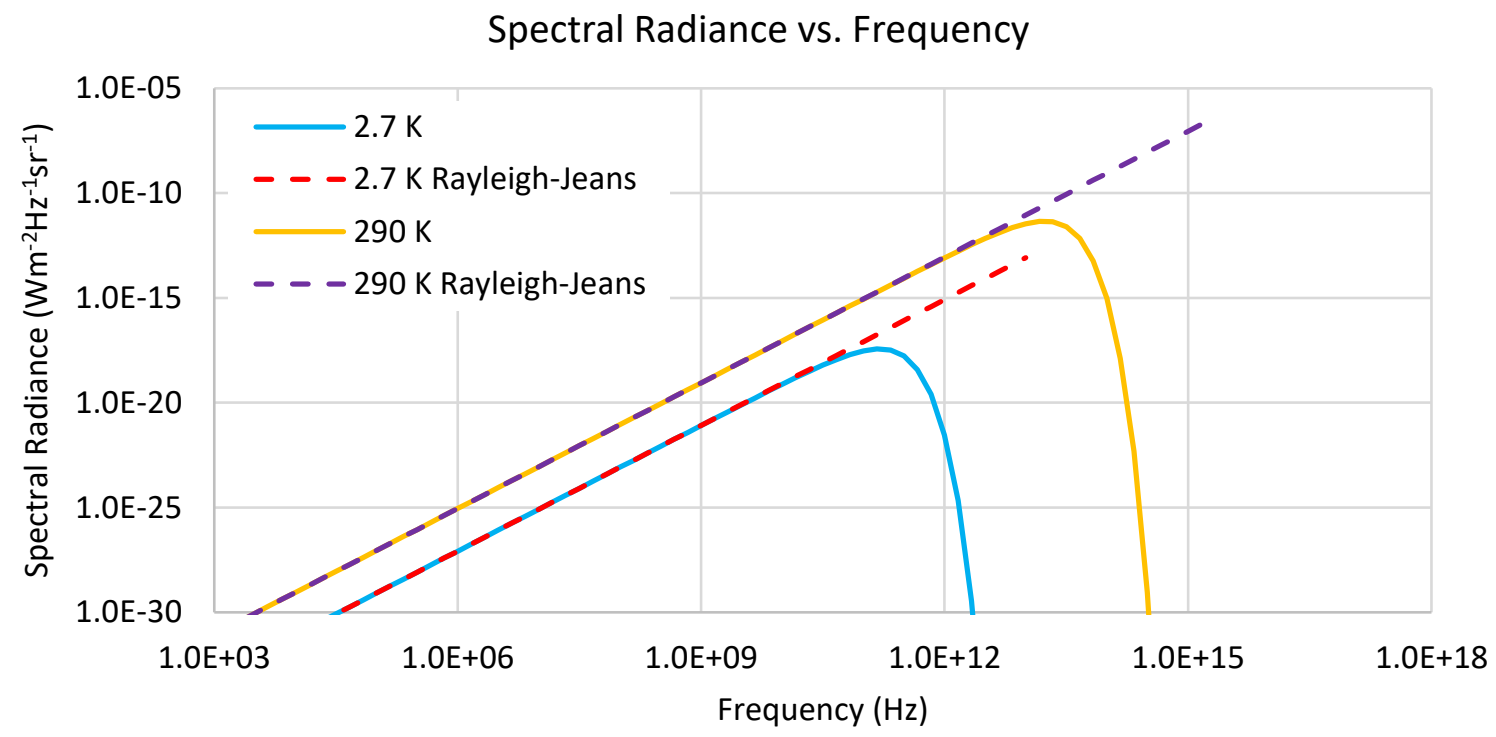

Figure 2.3: Spectral Radiance for Planck's Law compared to Rayleigh-Jeans Law

\subsubsection{Johnson-Nyquist Noise}

Similar to blackbody radiation, the motion of electrons caused by thermal energy within a resistor cause voltage fluctuations across it's terminals and is known as Johnson-Nyquist noise, named after the two engineers who studied this effect at bell labs [6] [7]. The mean-square noise voltage $\left(\overline{v_{T}^{2}}\right)$ across the terminals of a resistor with 
known resistance $(R)$ at a specific temperature and bandwidth $(B)$ is shown in Equation (2.7), and RMS voltage $\left(v_{R M S}\right)$ in Equation (2.8). Where $(k)$ is Boltzmann's constant.

$$
\begin{gathered}
\overline{v_{T}^{2}}=4 k T R B \\
v_{R M S}=\sqrt{\overline{\overline{v_{T}^{2}}}}=\sqrt{4 k T R B}
\end{gathered}
$$

For a matched impedance network with negligible reactance the transferable noise power becomes independent of resistance shown in Equation (2.9) and (2.10) where noise power is signified as $(N)$ [5] [6].

$$
\begin{gathered}
P_{N}=\frac{\left(v_{R M S}\right)^{2}}{4 R}=\frac{4 k T R B}{4 R} \\
P_{N}=N=k T B
\end{gathered}
$$

\subsubsection{Antenna Noise Temperature}

An antenna receives thermal noise power $\left(P_{A}\right)$ approximated by blackbody radiation from the environment within its field of view shown in Equation (2.11) where it's dependent on the antennas effective aperture $\left(A_{e}\right)$, spectral radiance $\left(L_{\lambda}\right)$, and bandwidth integrated over all angles, a solid angle $(\Omega)$ of $4 \pi[5]$ [6].

$$
P_{A}=\frac{1}{2} \int A_{e}(\theta, \phi) L_{\lambda} B d \Omega
$$

If the antenna is isotropic meaning its directivity is equal in all directions and it's placed in a cavity which acts as a uniform blackbody radiator, then Equation (2.11) can be simplified, shown in Equation (2.12).

$$
P_{A}=\frac{1}{2} A_{e} L_{\lambda} B \int d \Omega
$$


Using the approximation for spectral radiance for microwave and below, Equation (2.6) is incorporated with Equation (2.12) and integrating over all angles shown in Equation (2.13).

$$
P_{A}=2 \pi A_{e} B\left(\frac{2 k T}{\lambda^{2}}\right)=\frac{4 \pi A_{e} k T B}{\lambda^{2}}
$$

From experimentation, it's known the thermal noise power measured across the antennas terminals in a cavity is equal to a resistor at the same cavity temperature [5] [6]. Equation (2.13) for antenna noise power can be set equal to Equation (2.10) for Johnson-Nyquist noise.

$$
\begin{gathered}
P_{A}=\frac{4 \pi A_{e} k T B}{\lambda^{2}}=k T B=P_{N} \\
A_{e}=\frac{\lambda^{2}}{4 \pi}
\end{gathered}
$$

Equation (2.14) shows an antenna's effective aperture is quadratically related to wavelength, and Equation (2.15) shows received thermal noise power is wavelength or frequency independent. In conclusion the transferable thermal noise power received by an antenna for an impedance matched network is directly related to the environment temperature as seen by the antenna's beam pattern, shown in Equation (2.16) [5].

$$
T_{A}=\frac{1}{\Omega_{A}} \iint T_{E n v .}(\theta, \phi) P_{\text {Pattern }}(\theta, \phi) d \Omega
$$

\subsection{Effective System Noise Temperature}

A receiver's effective system noise temperature is a combination of the antenna's noise temperature, the antenna's effective feed noise temperature, and the receiver's noise temperature. Where the receiver's noise temperature is the cascaded 
combination of device noise temperatures starting from the first active device [4] [6] [7]. To understand the cumulative effect of this process a noisy two-port device is first discussed. Equation (2.18) shows the output thermal noise $\left(N_{\text {out }}\right)$ of a two-port device where $(G)$ is the power gain shown in Equation (2.17), $\left(N_{\text {in }}\right)$ is the input thermal noise, and $\left(N_{\text {int }}\right)$ is the internally generated noise power of the device [6] [7].

$$
\begin{gathered}
G=\frac{P_{\text {out }}}{P_{\text {in }}} \\
N_{\text {out }}=G\left(N_{\text {in }}+N_{\text {int }}\right)
\end{gathered}
$$

If the ratio of the signal-to-noise ratio (SNR) or CNR at the input is compared to the SNR/CNR at the output, then a noise factor of the two-port device can be evaluated.

$$
\begin{gathered}
F=\frac{S N R_{\text {in }}}{S N R_{\text {out }}}=\frac{P_{\text {in }} / N_{\text {in }}}{P_{\text {out }} / N_{\text {out }}}=\frac{N_{\text {out }}}{N_{\text {in }} G} \\
F=\frac{G\left(N_{\text {in }}+N_{\text {int }}\right)}{N_{\text {in }} G}=1+\frac{N_{\text {int }}}{N_{\text {in }}}=1+\frac{k T_{e} B}{k T_{0} B} \\
F=1+\frac{T_{e}}{T_{0}} \\
T_{e}=(F-1) T_{0}
\end{gathered}
$$

Equations (2.21) (2.22) give the noise factor $(F)$ or effective noise temperature $\left(T_{e}\right)$ of a two-port device [4] [6] [7]. It is common practice to always reference the noise factor of a device to a reference temperature $\left(T_{0}\right)$ of $290 \mathrm{~K}$. In addition, noise factor for RF components are commonly given in their logarithmic form called noise figure (NF) with units of $(\mathrm{dB})$ and the relation shown in Equation (2.23). 


$$
N F=10 \log _{10}(F)
$$

The two-port noise temperature formula can be applied to an attenuator which determines the effective noise temperature of the attenuator $\left(T_{A t t}\right)$. This includes antenna feed losses and passive devices evaluated for a specific operating frequency and at a physical temperature $\left(T_{P h y}\right)$, shown in Equation (2.24).

$$
T_{A t t}=(L-1) T_{P h y}
$$

When multiple devices are connected in series, whether they are active or inactive, the cascaded noise temperature is not simply the summation of them. The noise temperature of each stage is dependent on the gain or loss of each stage before it [6] [7]. Without presenting the derivation, the formula is shown in Equation (2.25) where $\left(G_{n}\right)$ is defined as the ratio of output power to input power for each stage and the numeric digit signifies the stage number.

$$
T_{\text {Total }}=T_{1}+\frac{T_{2}}{G_{1}}+\frac{T_{3}}{G_{1} G_{2}}+\cdots+\frac{T_{n}}{G_{1} \cdots G_{n-1}}
$$

The cascaded effect of noise temperatures can also be expressed as noise factors shown in Equation (2.26).

$$
F_{\text {Total }}=F_{1}+\frac{F_{2}-1}{G_{1}}+\frac{F_{3}-1}{G_{1} G_{2}}+\cdots+\frac{F_{n}-1}{G_{1} \cdots G_{n-1}}
$$

These cascaded formulas are commonly known as Friis formula for noise temperature and noise factor.

A formula for the effective system noise temperature $\left(T_{S}\right)$ of a receiver can now be described shown in Equation (2.27). The receiver itself can be thought of as a noisy 
two port device with an antenna as its source while neglecting antenna feed loss where $\left(T_{A}\right)$ is the antenna noise temperature and $\left(T_{R}\right)$ is the receiver's noise temperature as evaluated with Friis formula.

$$
T_{S}=T_{A}+T_{R}
$$

Friis formula for noise temperature is used to incorporate antenna feed losses which includes transmission lines and any passive devices before the first active device, shown in Equation (2.28) and Figure 2.4 where $\left(T_{F e e d}\right)$ is the effective noise temperature of the antenna feed.

$$
T_{S}=T_{A}+T_{\text {Feed }}+T_{R} L_{\text {Feed }}
$$

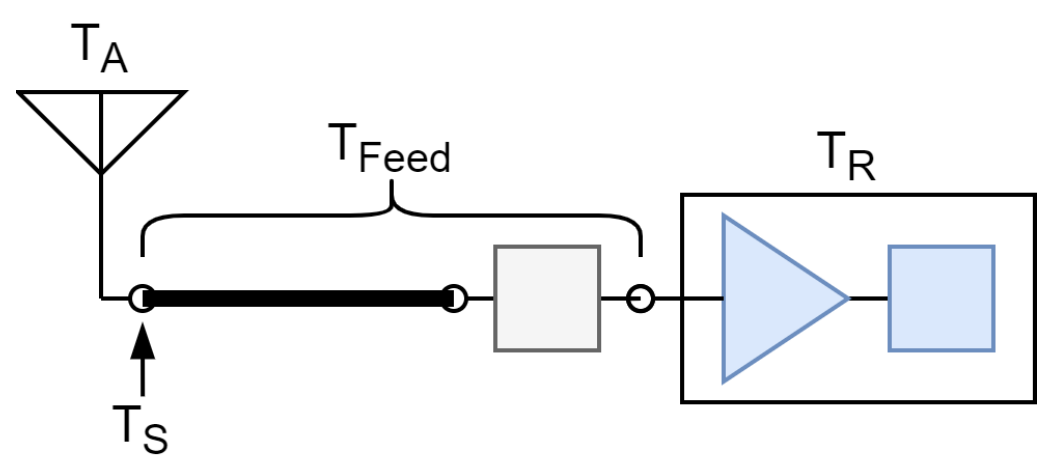

Figure 2.4: Effective System Noise Temperature Block Diagram

The effective noise temperature of an attenuator formula, Equation (2.24), is incorporated into Equation (2.28). The final effective system noise temperature of a receiver with an antenna as its source is shown in Equation (2.29) [6] [7]. This is the effective temperature of the system evaluated at the antenna's terminals which includes the source noise from the antenna and noise looking into the receiver from the antenna feed. 


$$
T_{S}=T_{A}+\left(L_{\text {Feed }}-1\right) T_{\text {Feed.Phy }}+T_{R} L_{\text {Feed }}
$$

The effective system noise temperature can also be thought of as the effective system noise power $\left(N_{S}\right)$ shown in Equation (2.30) where $\left(B_{N}\right)$ is the channel bandwidth.

$$
N_{S}=k T_{S} B_{N}
$$

\subsection{Digital Modulation}

For a digital communication system the required carrier-to-noise ratio (CNR) of the receiver is determined by the required energy per bit to noise power spectral density $\left(E_{b} / N_{0}\right)$ of a chosen modulation scheme. $\mathrm{E}_{\mathrm{b}} / \mathrm{N}_{0}$ is commonly used to analyze the modulation performance of a digital communication system due to being independent of data rate and bandwidth. Equation (2.31) shows how CNR correlates to $E_{b} / N_{0}$ where $\left(R_{b}\right)$ is data rate and $\left(B_{N}\right)$ is channel bandwidth of RF thermal noise [4].

$$
\frac{C}{N}=\frac{E_{b}}{N_{0}} \frac{R_{b}}{B_{N}}
$$

Demodulation scheme methods determine the relation of $\mathrm{E}_{\mathrm{b}} / \mathrm{N}_{\mathrm{o}}$ to bit-error-rate (BER) which is the ratio of average bit errors received to the total number of bits transmitted for an error rate probability. The derivation for $B E R$ vs. $E_{b} / N_{0}$ is outside of the scope of this paper. However, the formulas for binary phase-shift keying (BPSK), binary frequency-shift keying (BFSK) with a modulation index $\left(h_{m}\right)$ of 1.0, and minimumshift keying (MSK) which is BFSK with a modulation index of 0.5 are shown below in Equations (2.32) and (2.33) with their respective plots in Figure 2.5 below [7]. The CubeSat, OreSat1, will utilize up to $120 \mathrm{kbps}$ with a proposed modulation scheme of MSK for engineering uplink due to having better spectral efficiency compared to BPSK. 


$$
\begin{gathered}
B E R=Q\left(\sqrt{\frac{2 E_{b}}{N_{0}}}\right) \quad \text { for BPSK and MSK } \\
B E R=Q\left(\sqrt{\frac{E_{b}}{N_{0}}}\right) \quad \text { for BFSK, } h_{m}=1.0
\end{gathered}
$$

Theoretical BER vs. $\mathrm{E}_{\mathrm{b}} / \mathrm{N}_{0}$

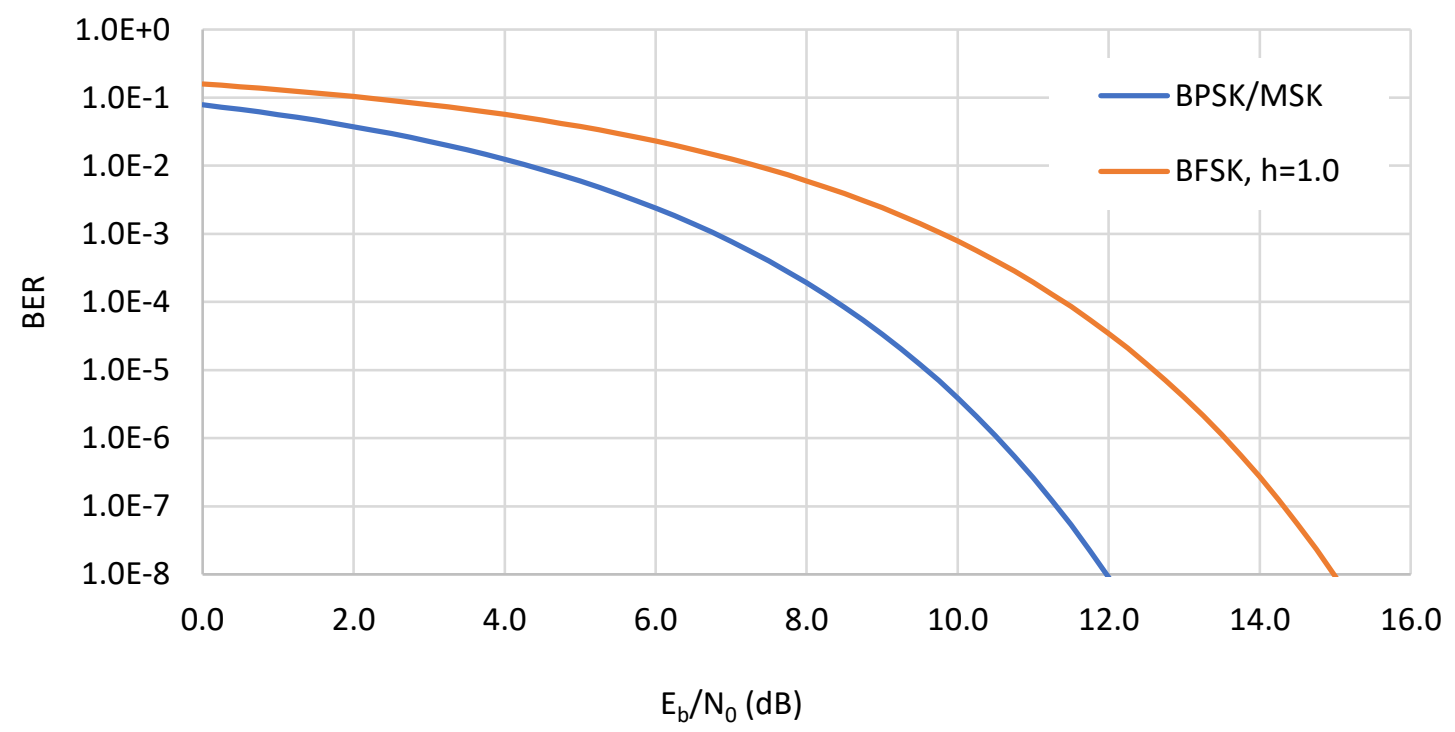

Figure 2.5: BER vs. Eb/No for BPSK, MSK, and BFSK modulation schemes

\subsection{Free-Space Path Loss}

The most significant loss of carrier power is from the physical space between the transmit and receive antennas called the free-space path loss (FSPL). This is caused by the geometric dispersion of power flux density of the electromagnetic radiation as it travels through space [4], shown in Equation (2.34) where $(d)$ is the distance traveled.

$$
L_{F S P L}=\left(\frac{4 \pi d}{\lambda}\right)^{2}=\left(\frac{4 \pi d f}{c}\right)^{2}
$$




\subsection{Link Budget Formula}

Link analysis for a LEO satellite can now be reviewed. From this point forward link analysis will employ a logarithmic scale for power which is most common for communication system analysis where the decibel $(\mathrm{dB})$ is used for relative power and the decibel-milliwatt $(\mathrm{dBm})$ is used for absolute power. These relations are shown in Equations (2.35) and (2.36).

$$
\begin{gathered}
P_{d B}=10 \log _{10}\left(P_{\text {ratio }}\right) \\
P_{d B m}=10 \log _{10}\left(P_{\text {Watt }}\right)+30
\end{gathered}
$$

As mentioned earlier, the required CNR at the receiver is the basis for a link budget. In addition, the excess of CNR at the receiver is defined as the link margin $\left(M_{L}\right)$ [4]. From Equation (2.4) link margin is incorporated, shown in Equation (2.37).

$$
M_{L}=\frac{C}{N_{S}}-\frac{C}{N_{R e q}}
$$

Since this is a digital communication system, the required CNR is transformed to the required $E_{b} / N_{0}$ by incorporating the $C N R$ to $E_{b} / N_{0}$ correlation, Equation (2.31), into (2.37). The receiver's thermal noise power is replaced by its definition for the effective system noise power of the receiver by incorporating Equation (2.30). An additional modulation implementation loss $\left(L_{M o d}\right)$ is introduced which contributes to the hardware and/or software's efficiency to demodulate and decode compared to theory; this parameter is included in the required $C N R$ to $E_{b} / N_{0}$ relationship. This expanded formula shown below in Equation (2.38) where $P_{R_{x}}$ is in $(\mathrm{dBm}), k$ in $(\mathrm{dBm} / \mathrm{Hz}-\mathrm{K})$, and all other in their logarithmic, decibel, form. 


$$
M_{L}=\left[P_{R_{x}}-k-T_{S}-B_{N}\right]-\left[\frac{E_{b}}{N_{0_{R e q}}}+R_{b}-B_{N}+L_{M o d}\right]
$$

The logarithmic form of the carrier power budget formula, based on Equation (2.3), is introduced and shown in Equation (2.39).

$$
P_{R_{x}}=P_{T_{x}}-L_{T_{x} \text {.Feed }}+G_{T_{x} A n t .}-L_{\text {Point }}-L_{F S P L}-L_{A t m o s .}+G_{R_{x} A n t .}
$$

The use of effective isotropic radiated power (EIRP) is a more common method of presenting the Earth station's transmitted power which incorporates losses after the transmit power amplifier and gain from the transmit antenna, shown in Equation (2.40). The simplified carrier power budget formula is shown in Equation (2.41).

$$
\begin{gathered}
E I R P=P_{T_{x}}-L_{T_{x} . \text { Feed }}+G_{T_{x} A n t .} . \\
P_{R_{x}}=E I R P-L_{\text {Point }}-L_{F S P L}-L_{\text {Atmos. }}+G_{R_{x} A n t .}
\end{gathered}
$$

The expanded power budget formula for received carrier power is incorporated into Equation (2.38) for link margin. For a digital communication system, the receiver and noise channel bandwidths are equal and therefore cancel in Equation (2.38). These modifications are shown in Equation (2.42).

$$
M_{L}=E I R P-L_{\text {Point }}-L_{F S P L}-L_{\text {Atmos. }}-L_{M o d}+G_{R_{x} A n t .}-k-T_{S}-R_{b}-\frac{E_{b}}{N_{0_{R e q}} .}
$$

Equation (2.42) shows the completed link budget formula evaluating link margin in logarithmic form. EIRP, FSPL, and the receiver's effective system noise temperature $\left(T_{S}\right)$ formulas are iterated in Equations (2.43) through (2.45) where the parameters inside the log brackets are in their non-logarithmic form. 


$$
\begin{gathered}
E I R P=P_{T_{x}}-L_{T_{x} . F e e d}+G_{T_{x} \text { Ant } .} \\
L_{F S P L}=10 \log _{10}\left[\left(\frac{4 \pi d f}{c}\right)^{2}\right] \\
T_{S}=10 \log _{10}\left[T_{A}+\left(L_{R_{x} . F e e d}-1\right) T_{\text {Feed.Phy }}+T_{R} L_{R_{x} \text {.Feed }}\right]
\end{gathered}
$$

\subsubsection{Alternative Method}

A popular alternative method of defining the effective system noise temperature $\left(T_{S}\right)$ exist which redefines the link margin formula $\left(M_{L}\right)[4]$ [8]. The effective system noise temperature can be evaluated with the effect of the antenna's feed loss removed from the system temperature. The feed loss is then incorporated as an additional parameter in the link margin formula. Figure 2.6 compares the two methods, where the location of $T_{S}$ is the original method described in the previous section and the location of $T_{S}^{*}$, in red, signifies the alternative method.

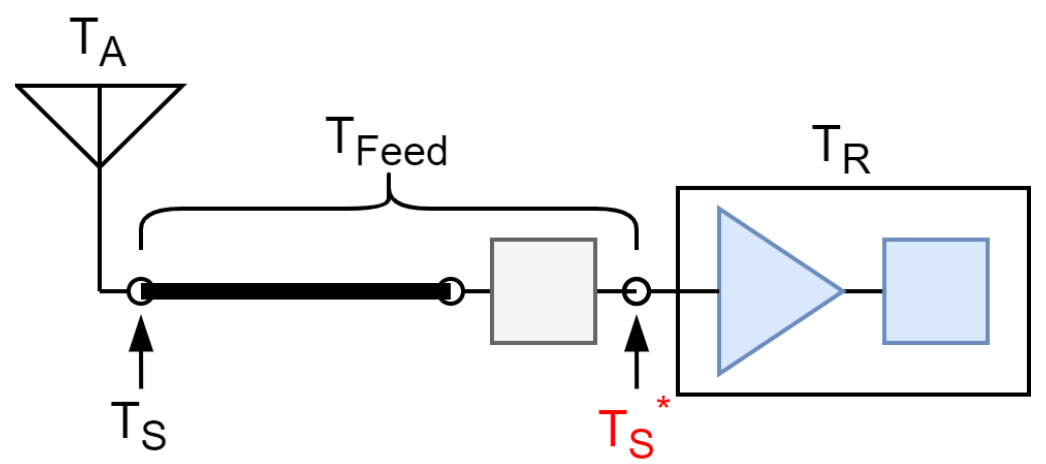

Figure 2.6: Alternative Effective System Noise Temperature Block Diagram

To remove the feed loss from the original formula, the loss is simply subtracted in the logarithmic form, shown in Equation (2.46).

$$
T_{S}^{*}=T_{S}-L_{R_{x} . F e e d}
$$


Transforming to ratio form and expanding original $T_{S}$ yields Equation (2.47).

$$
T_{S}^{*}=\frac{T_{A}+\left(L_{R_{x} . F e e d}-1\right) T_{F e e d . P h y}+T_{R} L_{R_{x} . F e e d}}{L_{R_{x} . F e e d}}
$$

The equation is simplified which yields the alternative effective system noise temperature independent of the antenna's feed loss, in ratio form, shown below [4].

$$
T_{S}^{*}=\frac{T_{A}}{L_{R_{x} \cdot \text { Feed }}}+\left(1-\frac{1}{L_{R_{x} \cdot \text { Feed }}}\right) T_{\text {Feed.Phy }}+T_{R}
$$

The alternative effective system noise temperature $\left(T_{S}^{*}\right)$ can now be incorporated back into the link margin formula with the additional antenna feed loss parameter, in red, shown in Equations (2.49) and (2.50) with the terms in brackets in their non-logarithmic form.

$$
\begin{gathered}
M_{L}=E I R P-L_{F S P L+\text { Point }+ \text { Atmos. }+ \text { Mod. }}-L_{R_{x} . \text { Feed }}+G_{R_{x} A n t .}-k-T_{S}^{*}-R_{b}-\frac{E_{b}}{N_{0_{R e q}}} \\
T_{S}^{*}=10 \log _{10}\left[\frac{T_{A}}{L_{R_{x} . F e e d}}+\left(1-\frac{1}{L_{R_{x} . F e e d}}\right) T_{\text {Feed.Phy }}+T_{R}\right]
\end{gathered}
$$

\subsubsection{Receiver Figure of Merit}

A very common performance figure of merit is the ' $G$ over $T^{\prime}(G / T)$ of a receiver, which is the ratio of the receiver's antenna gain to its effective system noise temperature [4] [8]. Either variation of the effective system noise temperature can be used, with or without the antenna feed losses excluded. However, if the alternative method is used which excludes the feed losses then they must be subtracted from the 
antenna's gain. Equations (2.51) and (2.52) show G/T with respect to both variations of the effective system noise temperature.

$$
\begin{gathered}
\frac{G}{T}=G_{R_{x} A n t .}-T_{S} \\
\frac{G}{T}=G_{R_{x} A n t .}-L_{R_{x} . \text { Feed }}-T_{S}^{*}
\end{gathered}
$$

Most link budgets employ the $\mathrm{G} / \mathrm{T}$ ratio instead of independently including the antenna's gain and effective system noise temperature. However, the link budgets shown in this paper will not employ G/T.

\subsection{CubeSat Link Budget}

The communication uplink for the LEO CubeSat, OreSat1, can now be analyzed. Many of the design choices for the satellite have been predetermined and are therefore fixed for this analysis including the receiver's antenna used and its associated gain, and antenna feed losses. In addition, pointing and polarization losses, and atmospheric and scintillation losses are predetermined, which will be reviewed.

The effective system noise temperature of the receiver has a lower attainable limit due to antenna noise temperature and design choices for the receiver's feed losses. In addition, the receiver's noise temperature is dependent on design choices and is also predetermined based on the L-band receiver design discussed in the following chapter. Therefore, the controllable parameters of the link budget are free-space path loss (FSPL) due to spacecraft elevation angle, data rate, and the effective isotropic radiated power (EIRP) from an Earth station. Using the link margin formula, either of these three parameters can be evaluated assuming the other two. The link margin 
formula from the previous section is shown in Equation (2.53). The link margin $\left(M_{L}\right)$ can be set to zero to determine the minimum or maximum value for a specific parameter.

$$
M_{L}=E I R P-L_{P o i n t}-L_{F S P L}-L_{\text {Atmos. }}+G_{R_{x} A n t .}-k-T_{S}-R_{b}-L_{M o d}-\frac{E_{b}}{N_{0_{R e q}}}
$$

\subsubsection{Minimum Receiver Power}

Before determining the three controllable parameters, the minimum received carrier power level can be evaluated first; this is the receiver's threshold level. Specifically, the carrier power level at the antenna's terminals which allows threshold operation with the receiver. The minimum received carrier power can be determined from the required CNR and adding the effective system noise power $\left(N_{S}\right)$ shown in Equation (2.54).

$$
P_{R_{x}}=\frac{C}{N}_{\text {Req. }}+N_{S}=\left[{\frac{E_{b}}{N_{0}}}_{\text {Req. }}+L_{M o d}+R_{b}-B_{N}\right]+\left[k+T_{S}+B_{N}\right]
$$

This equation is simplified and shown below along with the effective system noise temperature again.

$$
\begin{gathered}
P_{R_{x}}=k+T_{S}+R_{b}+L_{\text {Mod }}+\frac{E_{b}}{N_{0}} \\
T_{S}=10 \log _{10}\left[T_{A}+\left(L_{R_{x} . \text { Feed }}-1\right) T_{\text {Feed.Phy }}+T_{R} L_{R_{x} . \text { Feed }}\right]
\end{gathered}
$$

The effective system noise temperature $\left(T_{S}\right)$ is calculated first. A canted turnstile antenna was chosen for the CubeSat's L-band receiver. This type of antenna has a beam pattern similar to an isotropic model; therefore, being in LEO it effectively absorbs 
approximately half the Earth's radiation and half the cosmic microwave background due to the galactic center radiation being very low in L-band [4] [6]. This equates to an antenna temperature of about $150 \mathrm{~K}$. The CubeSat's antenna feed losses were predetermined to be $1.7 \mathrm{~dB}$, and a receiver noise temperature goal of $50 \mathrm{~K}$ is used. The effective system noise temperature evaluates to $368 \mathrm{~K}$ using a physical feed temperature of $300 \mathrm{~K}$. Table 2.1 summarizes these parameters.

\begin{tabular}{l|c|c|c} 
Parameter & Value & Units & Specification \\
\hline$T_{A}$ & 150 & $\mathrm{~K}$ & $1 / 2290 \mathrm{~K}+1 / 210 \mathrm{~K}(\max )$ \\
$\mathrm{T}_{\text {Feed.Phy }}$ & 300 & $\mathrm{~K}$ & Hot case \\
$\mathrm{T}_{\mathrm{R}}$ & 50.0 & $\mathrm{~K}$ & Goal \\
LRx.Feed & 1.48 & ratio & $0.7 \mathrm{~dB}$ backplane $+1.0 \mathrm{~dB}$ filter \\
\hline$T_{S}$ & 368 & $\mathrm{~K}$ & \\
& 25.7 & $\mathrm{~dB}-\mathrm{K}$ &
\end{tabular}

Table 2.1: Parameter summary of effective system noise temperature

The minimum received power can now be calculated. A data rate of $120 \mathrm{kbps}$ was desired, and minimum-shift keying (MSK) modulation scheme was chosen due to having a better spectral efficiency compared to BPSK, and lower required $\mathrm{E}_{\mathrm{b}} / \mathrm{N}_{0}$ compared to BFSK with an index of 1.0. The theoretical required $\mathrm{E}_{\mathrm{b}} / \mathrm{N}_{0}$ for $\mathrm{MSK}$ was calculated to be $8.4 \mathrm{~dB}$ for a BER of $10^{-4}$ from Equation (2.32). An estimated modulation implementation loss $\left(L_{M o d}\right)$ of $1.0 \mathrm{~dB}$ is initially assumed. The spacecraft's $(\mathrm{S} / \mathrm{C})$ minimum received power is evaluated using Equation (2.55). Three different data rates $\left(R_{b}\right)$ are evaluated and shown below in Table 2.2.

$$
P_{R_{x}}=k+T_{S}+R_{b}+L_{M o d}+{\frac{E_{b}}{N_{0_{R e q}}}}
$$




\begin{tabular}{l|c|ccc|c} 
& & $\mathrm{R}_{\mathrm{b}}=$ & $\mathrm{R}_{\mathrm{b}}=$ & $\mathrm{R}_{\mathrm{b}}=$ & \\
Parameter & Specification & $10 \mathrm{kbps}$ & $60 \mathrm{kbps}$ & $120 \mathrm{kbps}$ & Units \\
\hline $\mathrm{k}$ & - & -198.6 & -198.6 & -198.6 & $\mathrm{dBm} / \mathrm{Hz}-\mathrm{K}$ \\
$\mathrm{R}_{\mathrm{b}}$ & see heading & 40.0 & 47.8 & 50.8 & $\mathrm{~dB}-\mathrm{Hz}$ \\
$\mathrm{T}_{\mathrm{S}}$ & $368 \mathrm{~K}$ & 25.7 & 25.7 & 25.7 & $\mathrm{~dB}-\mathrm{K}$ \\
$\mathrm{E}_{\mathrm{b}} / \mathrm{N}_{0}$ Req. & $10^{-4} \mathrm{BER}$ & 8.4 & 8.4 & 8.4 & $\mathrm{~dB}$ \\
$\mathrm{~L}_{\text {Mod }}$ & estimate & 1.0 & 1.0 & 1.0 & $\mathrm{~dB}$ \\
\multicolumn{2}{l}{ Minimum Receiver Power: } & -123.5 & -115.7 & -112.7 & $\mathrm{dBm}$
\end{tabular}

Table 2.2: S/C Minimum receiver power

\subsubsection{Minimum Transmitter Power}

With the minimum received power determined, the three controllable parameters can be evaluated. Data rate was already determined with minimum received power which allows maximum FSPL and minimum EIRP from an Earth station to be evaluated. However, a minimum spacecraft elevation goal of $10^{\circ}$ is desired which determines the FSPL and hence a minimum EIRP remains to be evaluated. After determining EIRP a required transmitter antenna gain can be chosen in order to evaluate the minimum transmit power. Before this can be accomplished the FSPL must be computed first.

The distance required for free-space path loss (FSPL) is calculated by the provided formula shown below in Equation (2.58) which requires the Earth's radius $\left(R_{E}\right)$, altitude of the spacecraft $(H)$, and the spacecraft elevation $(\theta)$ in degrees with respect to the Earth station's horizon [4] [8]. This distance is commonly referred to as the slant range. This CubeSat will be launched from the International Space Station (ISS) with a starting altitude of around $400 \mathrm{~km}$. 


$$
\text { Slant Range: } \quad d=R_{E}\left\{\left[\frac{\left(H+R_{E}\right)^{2}}{R_{E}{ }^{2}}-\cos ^{2}(\theta)\right]^{1 / 2}-\sin \theta\right\}
$$

The slant range was evaluated for several elevations and summarized in Table 2.3.

\begin{tabular}{c|c|c} 
Elevation $\left(^{\circ}\right)$ & Slant Range $(\mathrm{km})$ & FSPL $(\mathrm{dB})$ \\
\hline 10 & 1,440 & 157.7 \\
30 & 739 & 151.9 \\
60 & 457 & 147.7 \\
90 & 400 & 146.5
\end{tabular}

Table 2.3: FSPL from various elevations for $400 \mathrm{~km} \mathrm{S/C} \mathrm{altitude}$

Minimum EIRP can now be evaluated from the link margin formula from Equation (2.53) while setting link margin to zero and rearranging, shown in Equation (2.59) and is simplified in (2.60). Pointing and polarization losses are estimated at around $3.0 \mathrm{~dB}$, and atmospheric and scintillation losses are low for L-band and estimated at $1.3 \mathrm{~dB}$ [4] [8]. In addition, the custom L-band canted turnstile on the spacecraft has an estimated gain of $1.4 \mathrm{dBi}$ from the back/bottom side beam.

$$
\begin{gathered}
E I R P=L_{\text {Point }}+L_{F S P L}+L_{\text {Atmos. }}-G_{R_{x} A n t .}+k+T_{S}+R_{b}+L_{\text {Mod }}+\frac{E_{b}}{N_{0_{\text {Req }}}} \\
E I R P=P_{R_{x}}+L_{\text {Point }}+L_{F S P L}+L_{\text {Atmos. }}-G_{R_{x} A n t .}
\end{gathered}
$$

Using Equation (2.60) the Earth station's (ES) minimum EIRP is evaluated for the same three different data rates $\left(R_{b}\right)$ and shown in Table 2.4 . 


\begin{tabular}{|c|c|c|c|c|c|}
\hline Parameter & Specification & $\begin{array}{c}R_{b}= \\
10 \mathrm{kbps}\end{array}$ & $\begin{array}{c}R_{b}= \\
60 \mathrm{kbps}\end{array}$ & $\begin{array}{c}R_{b}= \\
120 \mathrm{kbps}\end{array}$ & Units \\
\hline$P_{R x}$ & minimum & -123.5 & -115.7 & -112.7 & $\mathrm{dBm}$ \\
\hline $\mathrm{L}_{F S P L}$ & $1,440 \mathrm{~km}$ & 157.7 & 157.7 & 157.7 & $d B$ \\
\hline Lpoint & estimate & 3.0 & 3.0 & 3.0 & $d B$ \\
\hline LAtmos. & $10^{\circ}$ Elv. & 1.3 & 1.3 & 1.3 & $d B$ \\
\hline $\mathrm{G}_{\mathrm{Rx} . \text { Ant }}$ & Turnstile & $(-) 1.4$ & (-) 1.4 & (-) 1.4 & $\mathrm{dBi}$ \\
\hline \multirow{2}{*}{\multicolumn{2}{|c|}{ Minimum EIRP: }} & 37.1 & 44.9 & 47.9 & $\mathrm{dBm}$ \\
\hline & & 5.1 & 30.9 & 61.7 & W \\
\hline
\end{tabular}

Table 2.4: ES minimum Effective Isotropic Radiated Power (EIRP)

With EIRP evaluated, an antenna with appropriate gain for the Earth station's transmitter can be chosen. A data rate up to $120 \mathrm{kbps}$ is desired which requires $61.7 \mathrm{~W}$ of EIRP. An L-band quad helical antenna was chosen for the Earth station's transmitter which provides $16.0 \mathrm{dBi}$ of maximum gain with a beamwidth of $28^{\circ}$. The transmitter antenna feed losses including antenna mismatch loss were calculated at $1.8 \mathrm{~dB}$. The Earth station's minimum transmit power from the power amplifier can now be evaluated, shown in Table 2.5 .

\begin{tabular}{l|c|ccc|c} 
& & $\mathrm{R}_{\mathrm{b}}=$ & $\mathrm{R}_{\mathrm{b}}=$ & $\mathrm{R}_{\mathrm{b}}=$ & \\
Parameter & Specification & $10 \mathrm{kbps}$ & $60 \mathrm{kbps}$ & $120 \mathrm{kbps}$ & Units \\
\hline EIRP & minimum & 37.1 & 44.9 & 47.9 & $\mathrm{dBm}$ \\
$\mathrm{L}_{\text {Tx.Feed }}$ & calculated & 1.8 & 1.8 & 1.8 & $\mathrm{~dB}$ \\
$\mathrm{G}_{\text {Tx.Ant }}$ & Quad Helix & $(-) 16.0$ & $(-) 16.0$ & $(-) 16.0$ & $\mathrm{dBi}$ \\
\hline \multicolumn{2}{l}{ Minimum Transmit Power: } & 22.9 & 30.7 & 33.7 & $\mathrm{dBm}$ \\
& 0.19 & 1.17 & 2.34 & $W$
\end{tabular}

Table 2.5: ES minimum transmitter power

For a data rate of $120 \mathrm{kbps}$ and using a $16.0 \mathrm{dBi}$ Earth station antenna only 2.34 $W$ is required to close the link budget for a BER of $10^{-4}$. This is the absolute minimum required transmit power for a link margin of zero. It is important to note, if spacecraft 
orientation or pointing losses worsen then the link will not close. A positive link margin is commonly used to allow minor additional losses not foreseen. This concludes the initial theoretical evaluation of the CubeSat's L-band uplink analysis. It will be revised at the end of Chapter 4, Measurements and Results.

\section{Prototype Hardware}

The prototype hardware designed provided the ability to validate a communication link budget and serve as the primary receiver design on a real CubeSat mission, OreSat1. This chapter will provide mostly a high-level design concept of the Lband receiver for the spacecraft, and the transmitter designed specifically for validation. The primary objective of this thesis is the validation of a link budget and not necessarily the hardware design; therefore, design details will be brief with the exception to the receivers first stage low-noise amplifier (LNA).

\subsection{Receiver}

The CubeSat L-band receiver is based on a superheterodyne receiver (superhet) architecture which incorporates frequency conversion with a downconverter to shift a passband to a lower operating frequency. Frequency conversion is required due to using a sub-gigahertz receiver IC which will be discussed more in the following section. The superhet receiver architecture was chosen due to its simplicity and met the design requirements of conversion gain, spurious emission attenuation, and image rejection. 
An introduction to the superhet receiver architecture theory is discussed first, followed by the final CubeSat L-band receiver design.

\subsubsection{Superhet Architecture}

Figure 3.1 below, presents a minimal design superhet with four major sections which will be described starting from the antenna, left to right. This minimal design was the basis for the CubeSat L-band receiver. The first section, RF, is arguably the most important section due to having the most influence on the receiver's effective noise temperature. This was shown in section [2.3 Effective System Noise Temperature] with Friis formula for noise temperature. The first section also includes the initial filtering with a low insertion loss bandpass filter (BPF) before the first LNA. The second section, mixer, is where the frequency conversion takes place. A local oscillator (LO) drives a mixer which serves to create beat frequencies from the RF input and LO. The third section, intermediate frequency (IF), chooses the desired beat frequency produced from the mixer with a low-pass filter (LPF) for down conversion [9]. The fourth section is a digital receiver IC which employs its own mixer and LO. A modern receiver IC feeds the baseband to an analog-to-digital converter (ADC). In the digital domain it performs digital signal processing (DSP) to perform channel filtering, demodulation, decoding, and deframing before being sent to the first-in first-out (FIFO) buffer where a microcontroller can read from and utilize the data. 


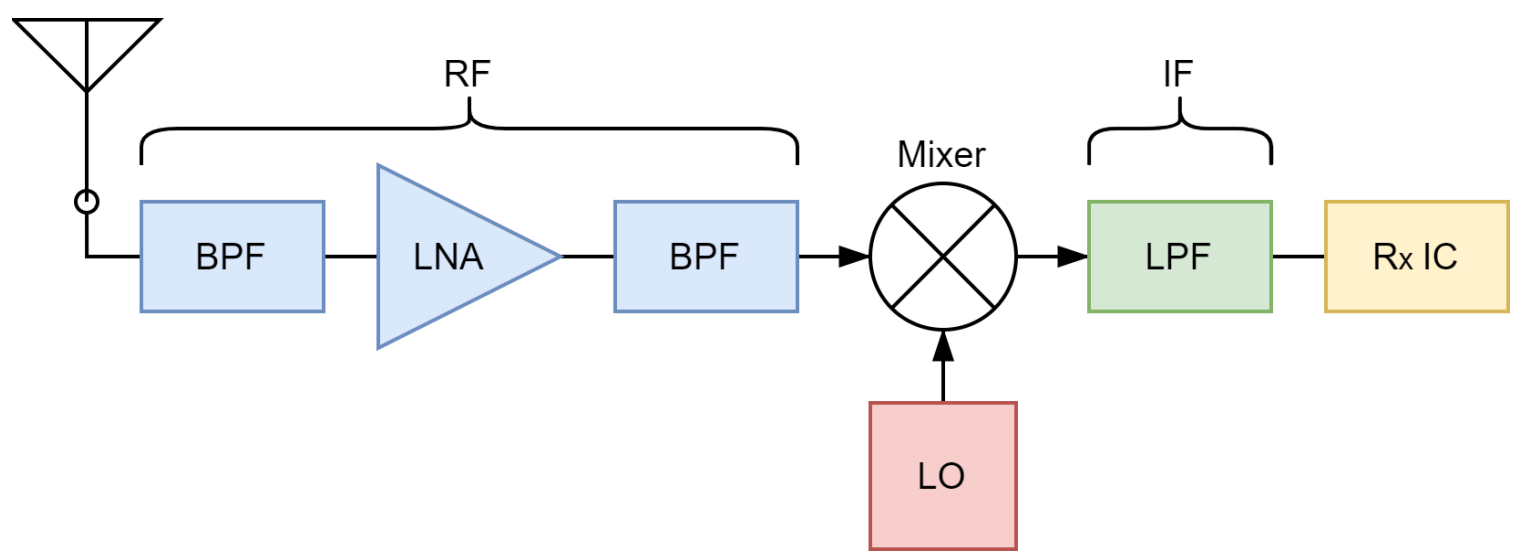

Figure 3.1: Minimal design superheterodyne block diagram

Frequency conversion is messy. Mixers are non-linear devices which create harmonic distortion of the LO which in turn cause intermodulation products. To mitigate unwanted beat frequencies and intermodulation products a few steps were taken. A LO frequency was chosen below and far enough away from the RF input frequency in order to maximize the separation of the lower IF $\left(\mathrm{IF}_{1}\right)$ from the $L O$ and $R F$, shown below in Figure 3.2. Narrow BPFs were chosen for the RF section to provide sufficient attenuation of neighboring bands and reject the potential image $\left(f_{3}\right)$ frequency. An image is the potential frequency on the RF input path which is exactly the lower IF difference $\left(\Delta \mathrm{IF}_{1}\right)$ mirrored from the LO. Frequency conversion of the image coincides with the lower IF (IF1). From the RF spectrum plot, Figure 3.2, it can be observed the RF BPFs, in blue, will sufficiently attenuate an image. In addition, the BPF just before the mixer acts to suppress thermal noise power in the image band from the LNA [10]. Finally, a steep LPF was chosen to attenuate the LO, RF, and $\mathrm{IF}_{2}$ frequencies. 


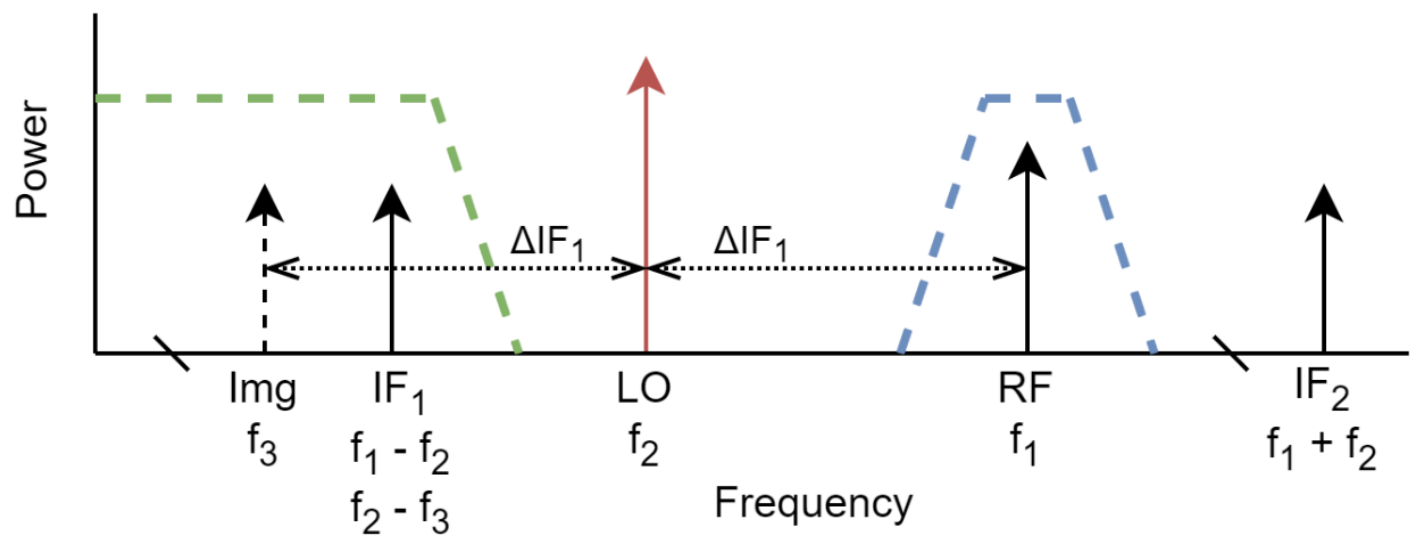

Figure 3.2: RF Spectrum of frequency down conversion

\subsubsection{CubeSat Receiver Design}

The final CubeSat L-band receiver design, shown in Figure 3.3, is not much more complex than the minimal design superhet. The RF section was expanded to include a secondary gain stage in order to further decrease the receiver's noise temperature and raise the carrier power level to the receiver IC. A GPS diplexer was inserted into the RF section in order receive GPS from the same L-band antenna. An additional BPF was inserted between the LNAs to assist with the first stage LNA stability which will be discussed in the following section.

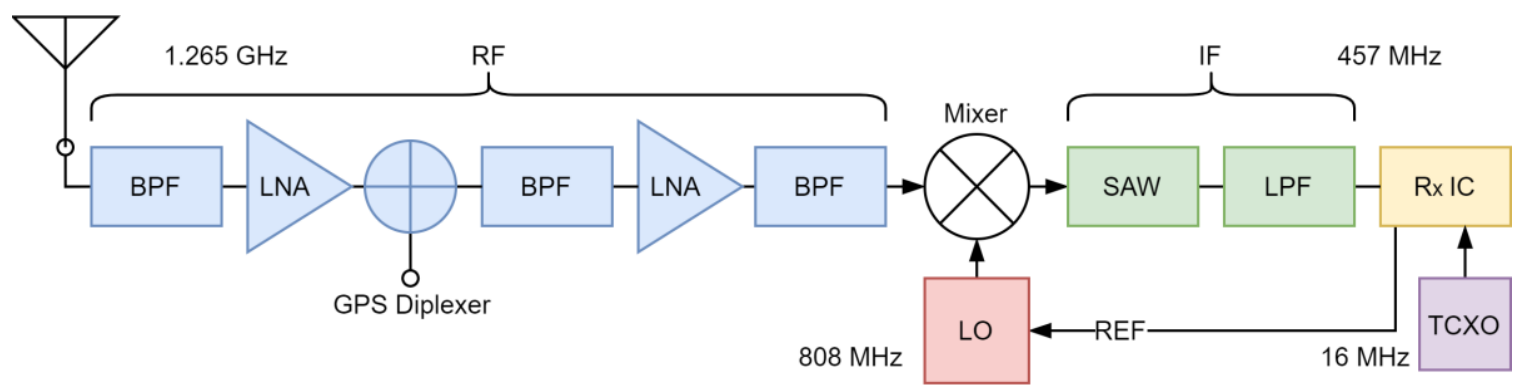

Figure 3.3: CubeSat superhet block diagram

A surface acoustic wave (SAW) filter was inserted into the IF section to provide better rejection of neighboring bands with a passband of $15 \mathrm{MHz}$. The SAW filter 
unfortunately does not provide excellent attenuation far away from the upper stop band; attenuation gradually decreases as frequency increases in the upper stopband. The LPF was kept in the IF section due to these SAW characteristics. A temperature controlled crystal oscillator (TCXO) was chosen as the primary reference for the whole receiver. It provides a $\pm 1.0 \mathrm{ppm}$ temperature stability over -40 to $+85^{\circ} \mathrm{C}$. The TCXO provides a $16 \mathrm{MHz}$ reference to the receiver IC which directly provides the same reference to the LO. Finally, a programmable low power RF synthesizer was chosen for the LO which provided a wide enough tuning range for flexibility of setting the optimal IF. Figure 3.3, shown above, provides high-level design of the CubeSat receiver design chosen. Detail component designs will not be discussed with the exception of the first LNA stage in the following section. The detailed block diagram of the CubeSat L-band receiver is shown in Figure 3.4. The total power consumption for the receiver was measured at $39 \mathrm{~mA}$ using a $3.3 \mathrm{~V}$ supply $(130 \mathrm{~mW})$ regardless of the data rate.

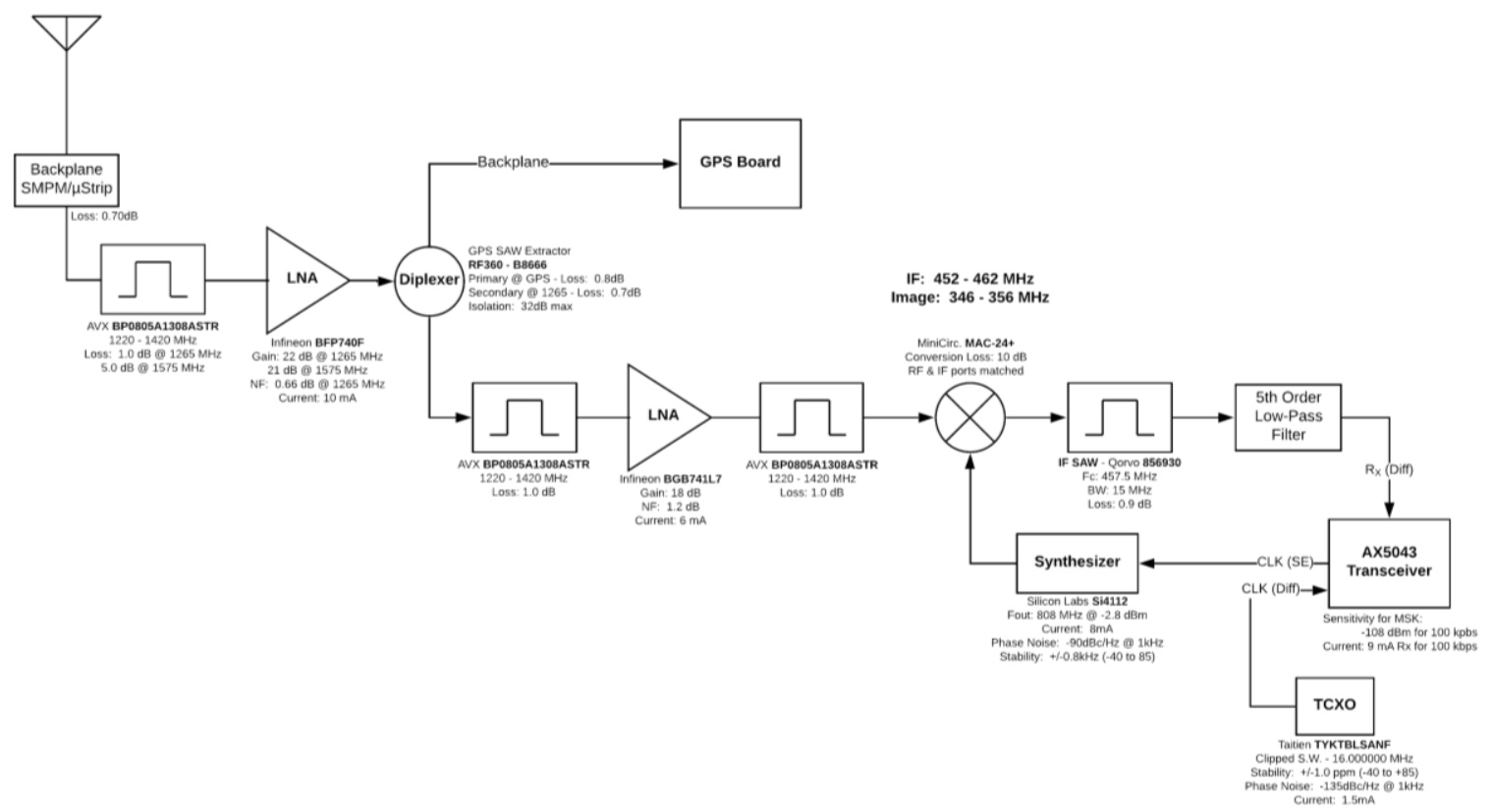

Figure 3.4: Detailed CubeSat receiver block diagram 
The CubeSat's L-band frequency range is set by the International

Telecommunication Union (ITU). Amateur radio satellites are allowed to use a small band of 1260 to $1270 \mathrm{MHz}$ for uplink only where each satellite has a coordinated frequency from the International Amateur Radio Union (IARU). The receiver IC has two small RF tuning ranges in UHF with ranges wide enough and far enough away from 1265 MHz. Table 3.1 shows a summary of the allowable LO operating range which corresponds to the receiver's operating ranges for an RF operating range of 1260 to $1270 \mathrm{MHz}$. A low power RF synthesizer operating in the $800 \mathrm{MHz}$ range was available, therefore operating in the receiver's lower range provided enough separation between IF, LO, and RF frequencies.

\begin{tabular}{c|c} 
Receiver IC Range (IF) & LO Allowable Range \\
\hline $400-525 \mathrm{MHz}$ & $860-745 \mathrm{MHz}$ \\
$800-1050 \mathrm{MHz}$ & $460-220 \mathrm{MHz}$
\end{tabular}

Table 3.1: Receiver LO and IF tuning ranges

An IF center frequency of $457 \mathrm{MHz}$ was chosen, $452-462 \mathrm{MHz}$ tunable, with an 808 MHz fixed LO, shown in Table 3.2. This operating range was chosen due to a SAW filter being available and a harmonic of the TCXO reference not existing in the tunable IF range.

\begin{tabular}{c|c}
$\begin{array}{c}\text { Mixer In } \\
(\mathrm{RF}-\mathrm{LO})\end{array}$ & $\begin{array}{c}\text { Mixer Out / } \\
\text { Receiver IC Input (IF) }\end{array}$ \\
\hline $1260-808 \mathrm{MHz}$ & $452 \mathrm{MHz}$ \\
$1270-808 \mathrm{MHz}$ & $462 \mathrm{MHz}$
\end{tabular}

Table 3.2: Receiver fixed LO and IF operating range 


\subsubsection{CubeSat Receiver Construction}

All receiver components from the block diagram were designed and assembled by the author of this paper as separate evaluation boards in order to independently test and measure them. Schematics and layouts were designed with EAGLE CAD for 4-layer printed circuit boards (PCB), and they were manufactured by OSH Park. For RF signal paths, microstrips were designed and terminated with end-launch SMA connectors. Board specifications are discussed further in section [4.1 Hardware ]. Many of the evaluation boards are shown in Figure 3.5 below, and the completed prototype L-band receiver is shown in Figure 3.6.

In addition, the 4-layer stackup OSH Park offers is designed with Isola FR408HR substrate and has a relative permittivity of 3.69 at $1 \mathrm{GHz}$. The copper layer height between the outer layers is specified at 6.7 mils $(0.17 \mathrm{~mm})$. Based on microstrip calculations the optimal $50 \Omega$ microstrip impedance should be $0.34 \mathrm{~mm}$. It was found through time-domain reflectometry (TDR) the true $50 \Omega$ impedance was close to 0.38 $\mathrm{mm}$. 


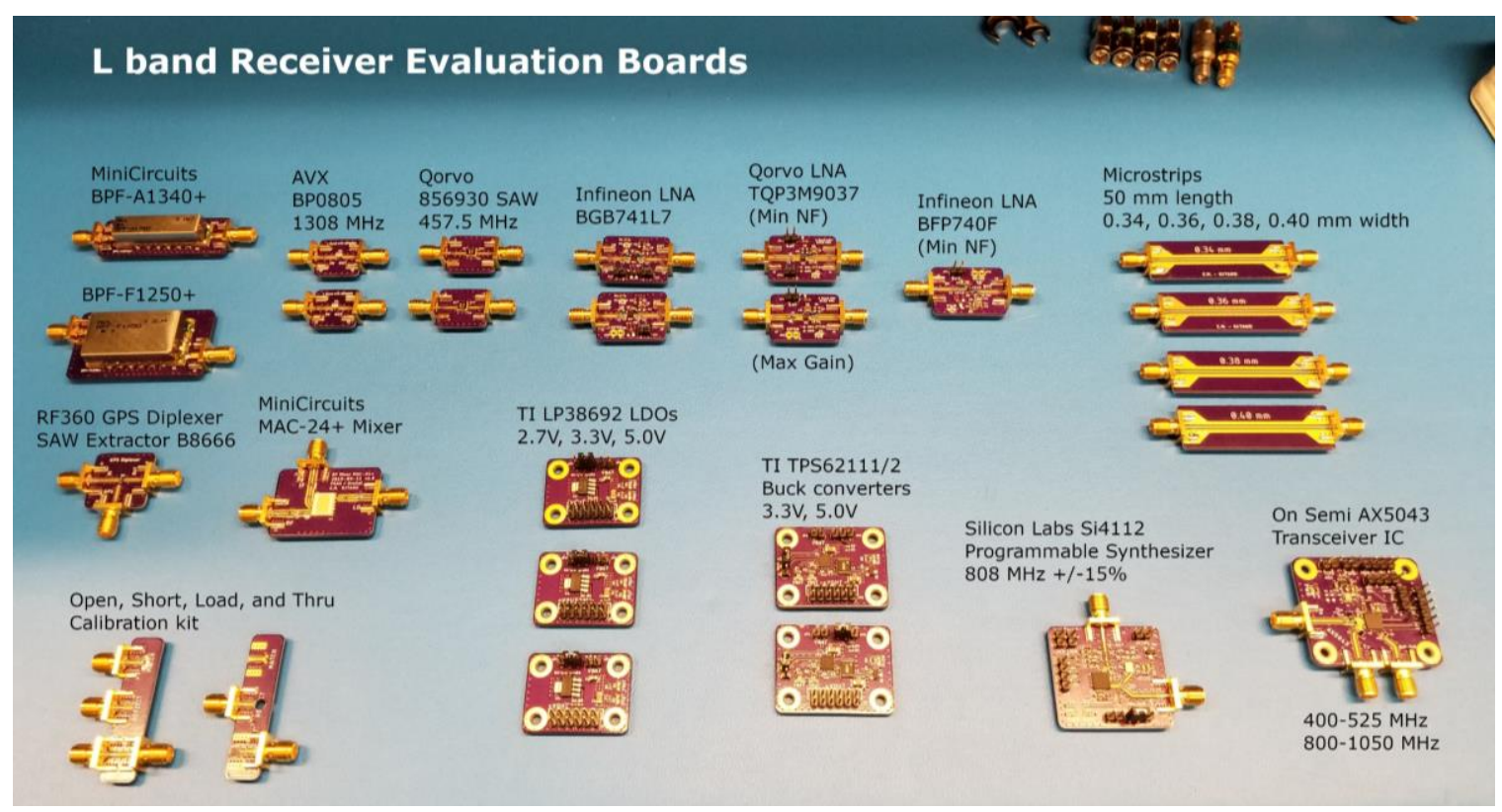

Figure 3.5: L-band receiver evaluation boards designed and assembled by author

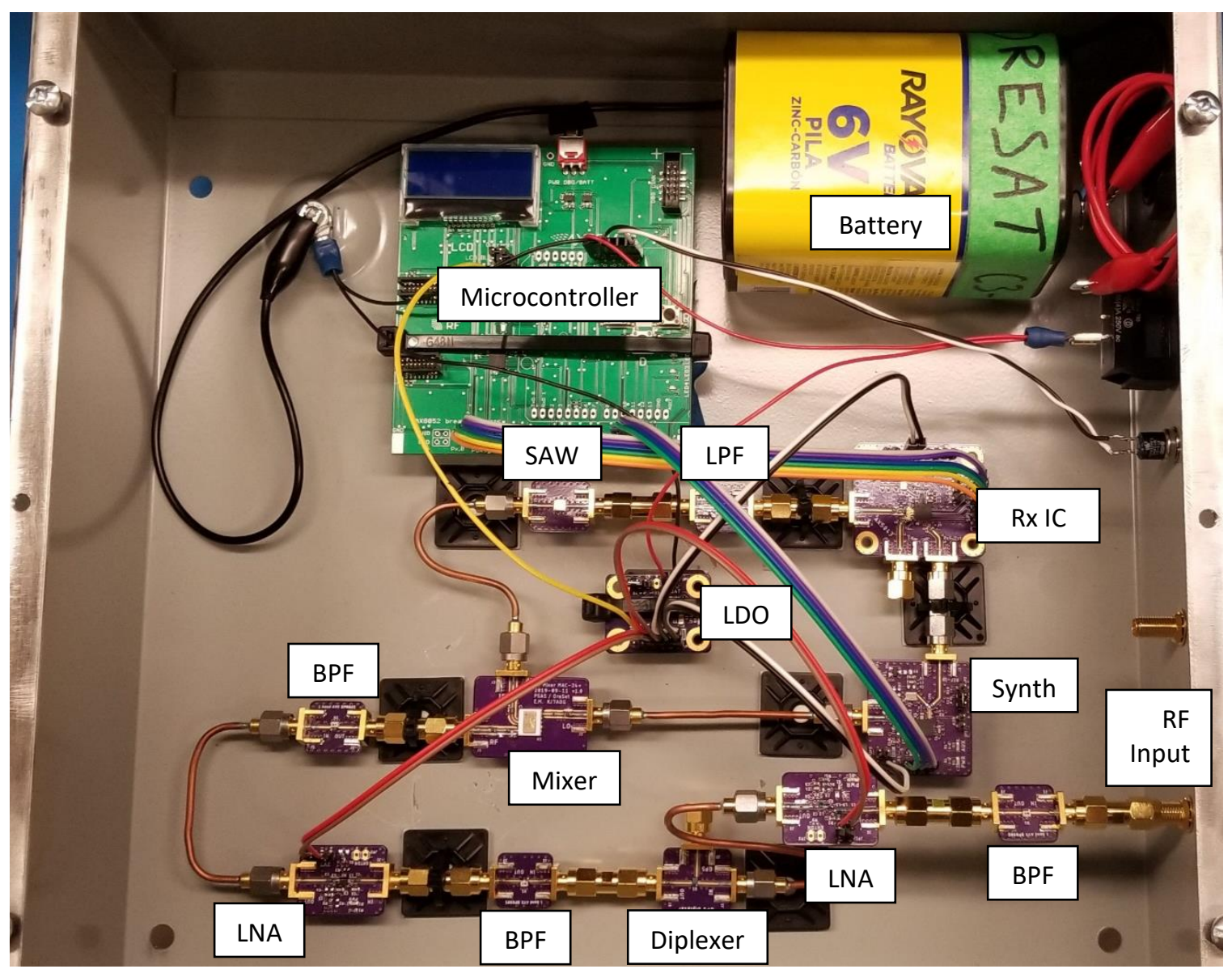

Figure 3.6: Prototype L-band receiver (purple boards) in steel box. Designed and assembled by author. 


\subsubsection{First Stage LNA Design}

The first stage low-noise amplifier (LNA) has the most influence on the receiver's effective noise temperature. The goal was to achieve at least a $50 \mathrm{~K}$ noise temperature ( $\leq 0.69 \mathrm{~dB}$ NF) LNA design which consumed $10 \mathrm{~mA}$ or less at $2.7 \mathrm{~V}$. This is possible for a satellite LNA due to the input power levels being very low. A high output $1 \mathrm{~dB}$ compression point (OP1dB) was not required, therefore a high quiescent current is not required. To achieve these LNA characteristics an RF bipolar-junction transistor (BJT) from Infineon Technologies was chosen with design goals to maximize transducer gain $\left(\left|S_{21}\right|^{2}\right)$, while minimizing noise figure (NF), and quiescent current. The prototype first stage L-band LNA is shown in Figure 3.7.

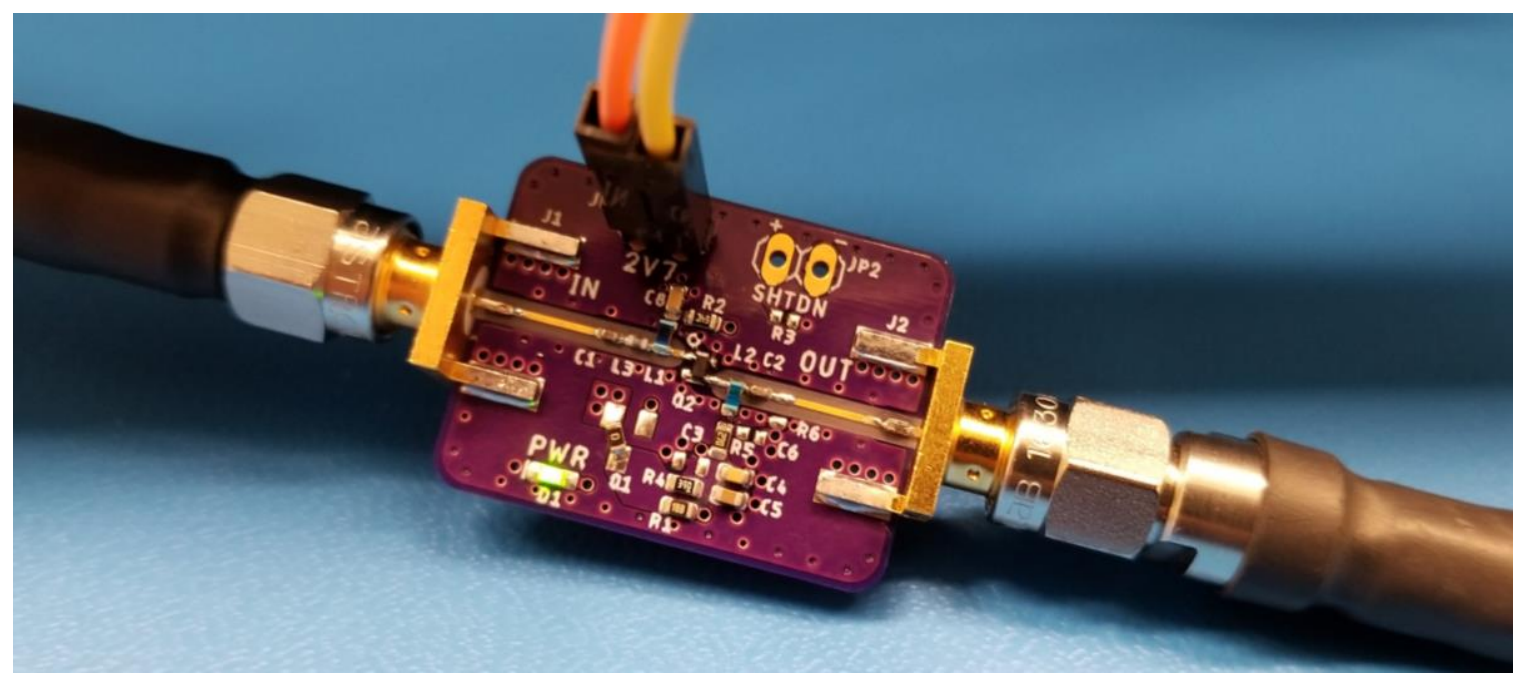

Figure 3.7: First stage L-band LNA with Infineon Technologies BFP74OF RF BJT. Designed and assembled by author.

To design such an LNA either requires many revisions to fine tune characteristics or the use of design simulation software with a model of the device's intrinsic characteristics from the manufacturer, the latter was chosen. Simulation software called Advanced Design System (ADS) from Keysight Technologies was used with the de- 
embedded two-port scattering parameters (S2P) file from Infineon for the BJT semiconductor device. This characterization file also includes noise figure data. Before further design details are discussed, a brief introduction of scattering parameters (S parameters) are reviewed.

S parameters are used to characterize the incident and reflected electromagnetic travelling waves at each port instead of voltages and currents in RF/microwave devices/networks [11] [12]. These are defined as reflection and transmission coefficients of a network while being terminated with its characteristic impedance, specifically the system's operating impedance, usually $50 \Omega$. The definition of reflection coefficient along with incident and reflected waves for a 1-port network is first introduced and shown in Equations (3.1), (3.2), and (3.3).

$$
\begin{array}{lc}
\text { Incident Wave: } & a(x) \sqrt{Z_{0}}=V^{+}(x)=A e^{-j \beta x} \\
\text { Reflected Wave: } & b(x) \sqrt{Z_{0}}=V^{-}(x)=B e^{j \beta x} \\
\text { Reflection Coefficient: } & S_{11}=\Gamma(x)=\frac{V^{-}(x)}{V^{+}(x)}
\end{array}
$$

For a two-port network, an incident wave on one port will be partially reflected and partially transmitted. This allows for an updated definition of reflection coefficient and introduces the transmission coefficient. Equations (3.4) and (3.5) show these relations.

$$
\begin{aligned}
& S_{11} a_{1}+S_{12} a_{2}=b_{1} \\
& S_{21} a_{1}+S_{22} a_{2}=b_{2}
\end{aligned}
$$


All of the two-port S parameters can now be defined. Equations (3.6) through (3.9) summarize the four S parameters where the terms $S_{i j}$ defines the S parameter with $i$ port reflected/transmitted wave and $j$-port incident wave.

$$
\begin{array}{lr}
\text { Input Reflection Coefficient: } & S_{11}=\left.\frac{b_{1}}{a_{1}}\right|_{a_{2}=0} \\
\text { Output Reflection Coefficient: } & S_{22}=\left.\frac{b_{2}}{a_{2}}\right|_{a_{1}=0} \\
\text { Forward Transmission Coefficient: } & S_{21}=\left.\frac{b_{2}}{a_{1}}\right|_{a_{2}=0} \\
\text { Reverse Transmission Coefficient: } & S_{12}=\left.\frac{b_{1}}{a_{2}}\right|_{a_{1}=0}
\end{array}
$$

Design details of the first stage L-band LNA can now proceed.

The first step to design an LNA is to analyze its intrinsic characteristic $\mathrm{S}$ parameters. An operating point of $10 \mathrm{~mA}$ with a $\mathrm{V}_{\mathrm{CE}}$ of $2.0 \mathrm{~V}$ was chosen. The manufacturer provides de-embedded S parameter data for the most common operating points of the device. When analyzing S parameters in ADS with the manufacturer's device, $\mathrm{DC}$ biasing is independent of the $\mathrm{S}$ parameter analysis and $\mathrm{DC}$ biasing is not required to be setup by the user. Figure 3.8 below, presents the initial ADS schematic with the Infineon BFP740F RF BJT biased with a quiescent current of $10 \mathrm{~mA}$ and without matching networks. 


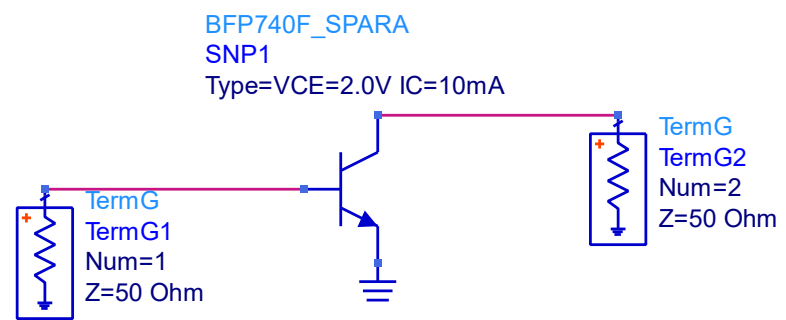

Figure 3.8: ADS schematic for initial device characterization of Infineon BFP74OF RF BJT

The Smith chart shown below in Figure 3.9 displays the devices input and output reflection coefficients, $S_{11}$ and $S_{22}$ respectively, over a frequency sweep of $200 \mathrm{MHz}$ to 6.0 GHz with the markers at $1265 \mathrm{MHz}$ for the CubeSat's L-band center operating frequency. A Smith chart is a polar plot of reflection coefficient, $S_{11}$ or $S_{22}$ for a two-port device, with constant resistance circles emanating from the right side starting at infinity and ending at zero on the left, along with constant reactance curves emanating from the plot's horizon with inductive curves on the upper hemisphere and capacitive curves on the lower. The magnitude of reflection coefficient, $\left|S_{11}\right|$ or $\left|S_{22}\right|$, is the distance ratio from the Smith chart's center. The center is the characteristic impedance of the system and is $50 \Omega$ for this system. It can be observed that the device requires matching networks for maximum transferable power to be achieved. 


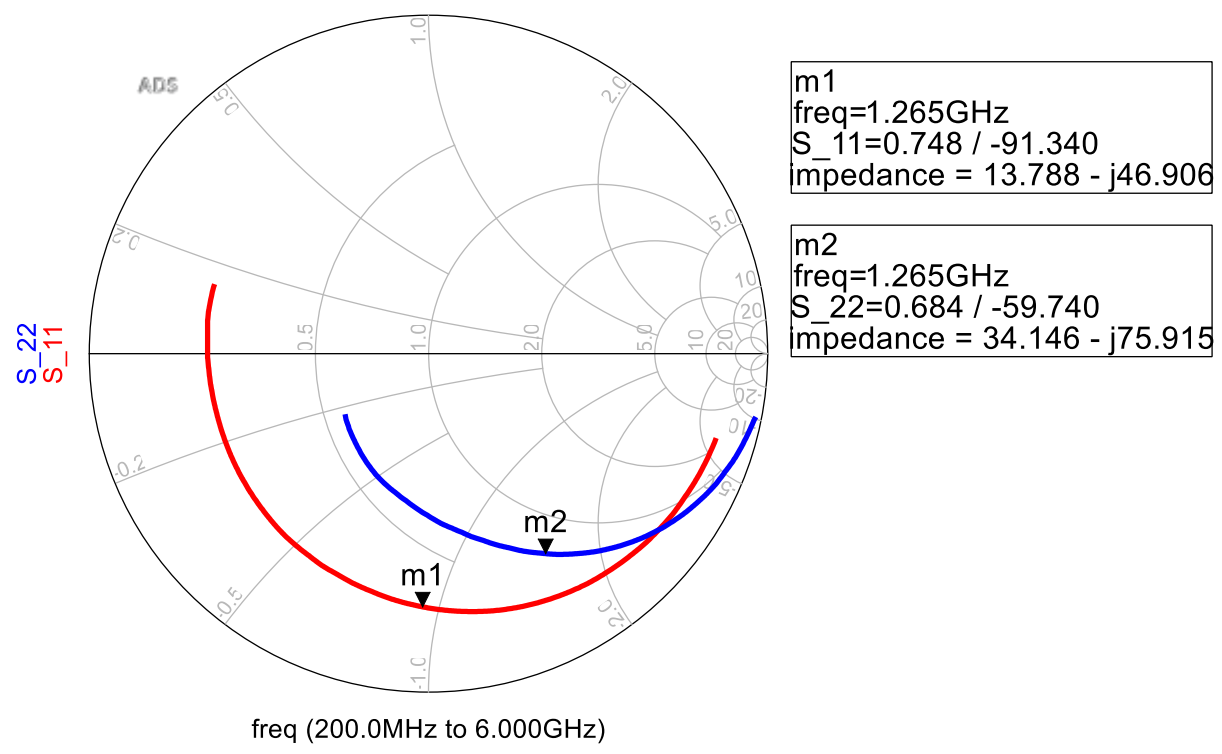

Figure 3.9: Smith chart of BFP74OF without matching

The magnitude plot of reflection and transmission coefficients is shown in Figure 3.10. The plot is in decibel form which is defined as the ratio of reflected or transmitted to incident powers instead of voltage waves. This is defined in Equation (3.10).

$$
S_{i j}(d B)=\frac{P_{\text {ref } / \text { tran }}}{P_{\text {incident }}}=10 \log _{10}\left(\left|S_{i j}\right|^{2}\right)
$$
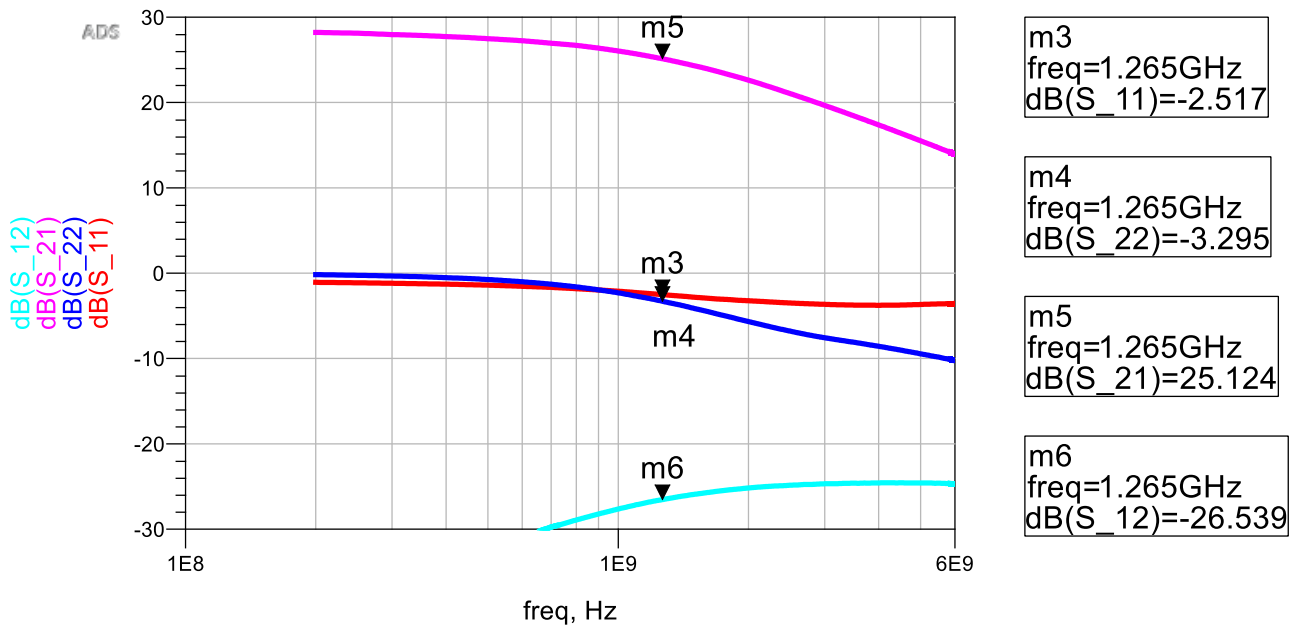

Figure 3.10: S parameter magnitude plot of BFP74OF without matching 
Using the Smith chart, the optimal source and load impedances can be plotted which are the complex conjugate reflection coefficients. Points on the Smith chart can be plotted for optimal source and load impedances evaluated at the operating frequency of $1265 \mathrm{MHz}$. These points represent the maximum unilateral transducer power gain which is the maximum transferable power gain for a transistor with $S_{12}$ approximately equal to zero. In addition, constant gain circles can be plotted which show source and load impedance target regions. The circle edges are the power gain or loss with respect to the transistor's transducer gain, $\left|S_{21}\right|^{2}$ in $\mathrm{dB}$, if the source or load impedance were at those locations. Figure 3.11 shows the Smith charts with optimal source and load impedances along with their constant gain circles.
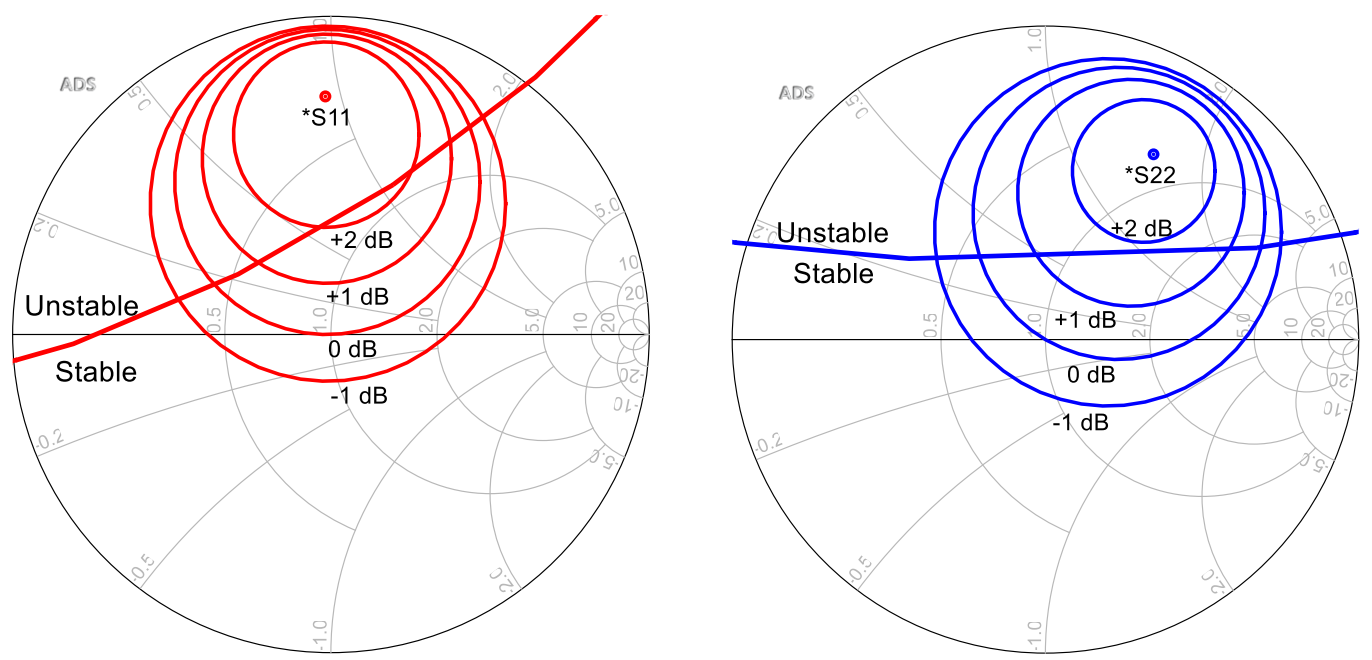

Figure 3.11: Smith charts of optimal source/load, constant gain circles, and stability circles evaluated at $1265 \mathrm{MHz}$ for BFP74OF w/o matching. Source (left, red) and Load (right, blue).

Figure 3.11 also shows the edges of stability circles evaluated at $1265 \mathrm{MHz}$. The stability circles' edges define the boundary between stable and unstable regions on the Smith chart for source and load impedances, where an unstable transistor design 
oscillates. The optimal source and load impedances evaluated at $1265 \mathrm{MHz}$ for the BFP740F fall within the unstable region. This negates the transistor's ability to reach maximum unilateral transducer power gain.

The first step in this transistor design was to add stability improvements to allow the transistor to reach maximum unilateral transducer power gain in a conditionally stable state. Emitter degeneration and output shunt resistance were chosen for the stability improvements. Figure 3.12 shows the initial conditionally stable transistor design in ADS with short microstrips on the base and collector ports as well. In addition, Figure 3.13, below, shows the updated magnitude plots, and Figure 3.14 shows the associated Smith charts for optimal source and load impedances with constant gain circles and stability circles for the stability improved initial design.

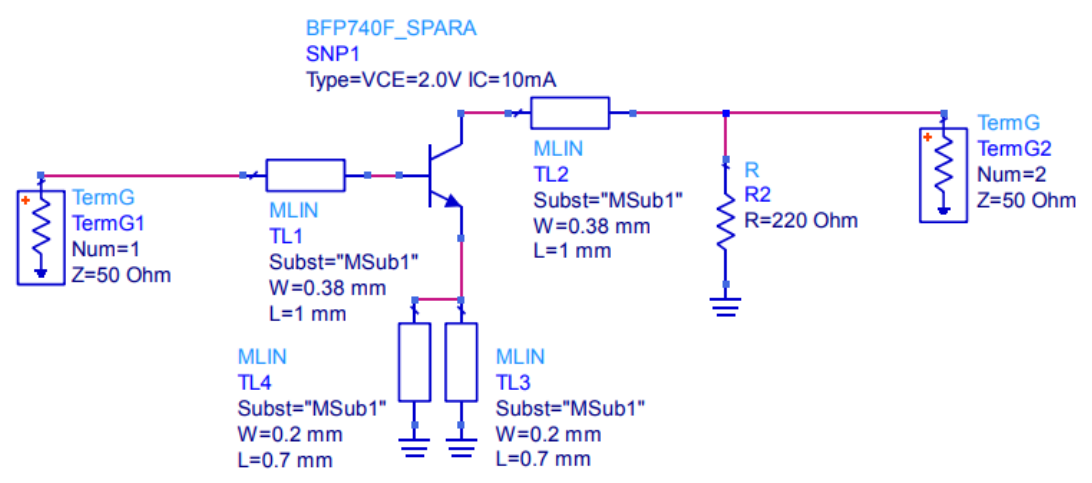

Figure 3.12: ADS schematic for stability improved initial device of Infineon BFP74OF RF BJT with stability improvements added. 

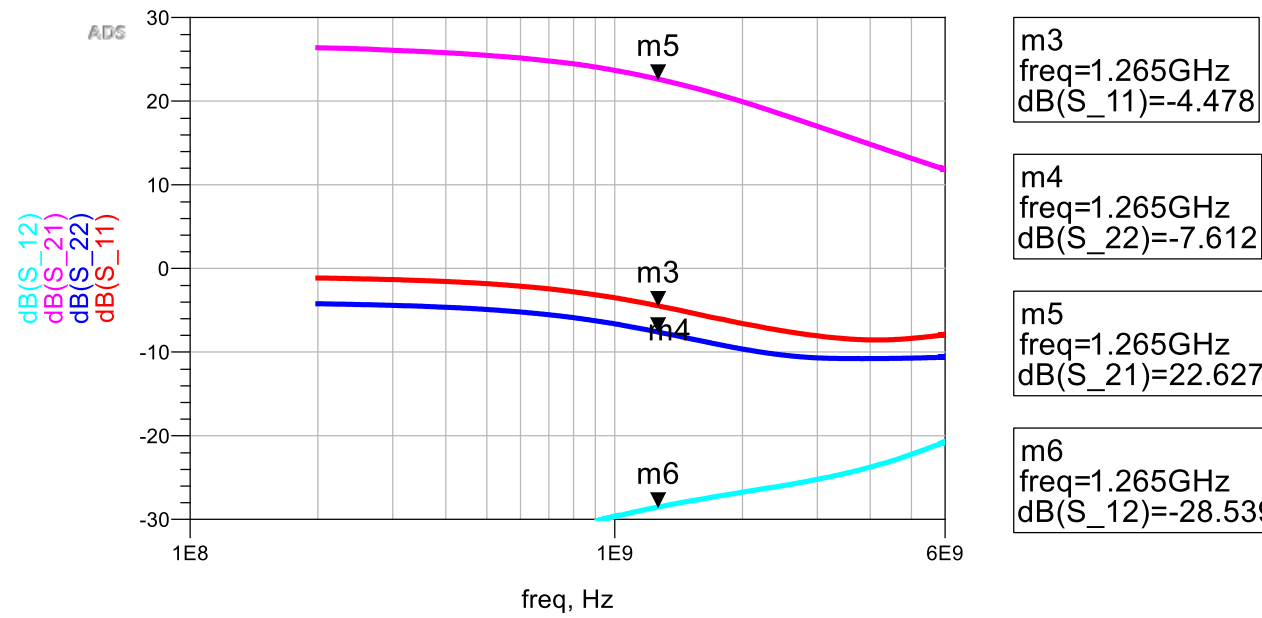

$\mathrm{m} 4$

freq $=1.265 \mathrm{GHz}$

$\mathrm{dB}\left(\mathrm{S} \_22\right)=-7.612$

$\mathrm{m} 5$

freq $=1.265 \mathrm{GHz}$

$\mathrm{dB}\left(\mathrm{S} \_21\right)=22.627$

$\mathrm{m} 6$

freq $=1.265 \mathrm{GHz}$

$\mathrm{dB}\left(\mathrm{S} \_12\right)=-28.539$

Figure 3.13: S parameter magnitude plot of BFP74OF with stability improvements added.
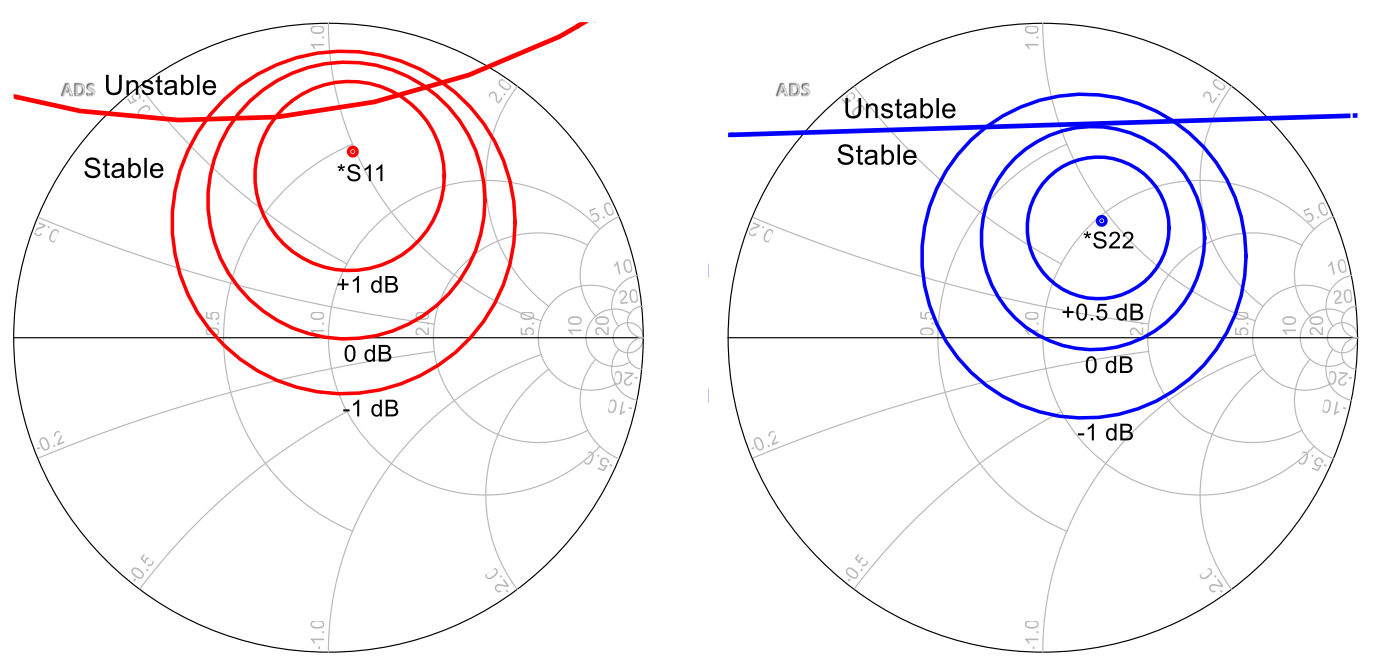

Figure 3.14: Smith charts of optimal source/load, constant gain circles, and stability circles evaluated at $1265 \mathrm{MHz}$ for BFP740F with stability improvements added. Source (left, red) and Load (right, blue).

The effects of the stability improvements can also be analyzed by comparing the stability factor or K-factor of the transistor with and without the stability improvements. $\mathrm{K}$-factor is calculated with the below formula in Equation (3.11). If $\mathrm{K}$ is less than one, then the transistor design is potentially unstable dependent on the impedance of the source and load matching networks. If the design is stable with a $\mathrm{K}$ value less than one, then it is defined as conditionally stable. If $\mathrm{K}$ is greater than one, the design could 
theoretically use any source and load matching networks and still be stable. This would be defined as an unconditionally stable state. The magnitude of K corresponds to how much of the stability circle's unstable region is occupying the Smith chart. Figure 3.15 shows stability factor plots of the initial transistor design before and after the stability improvements.

$$
K=\frac{1-\left|S_{11}\right|^{2}-\left|S_{22}\right|^{2}+\left|S_{11} S_{22}-S_{12} S_{21}\right|^{2}}{2\left|S_{12} S_{21}\right|}
$$
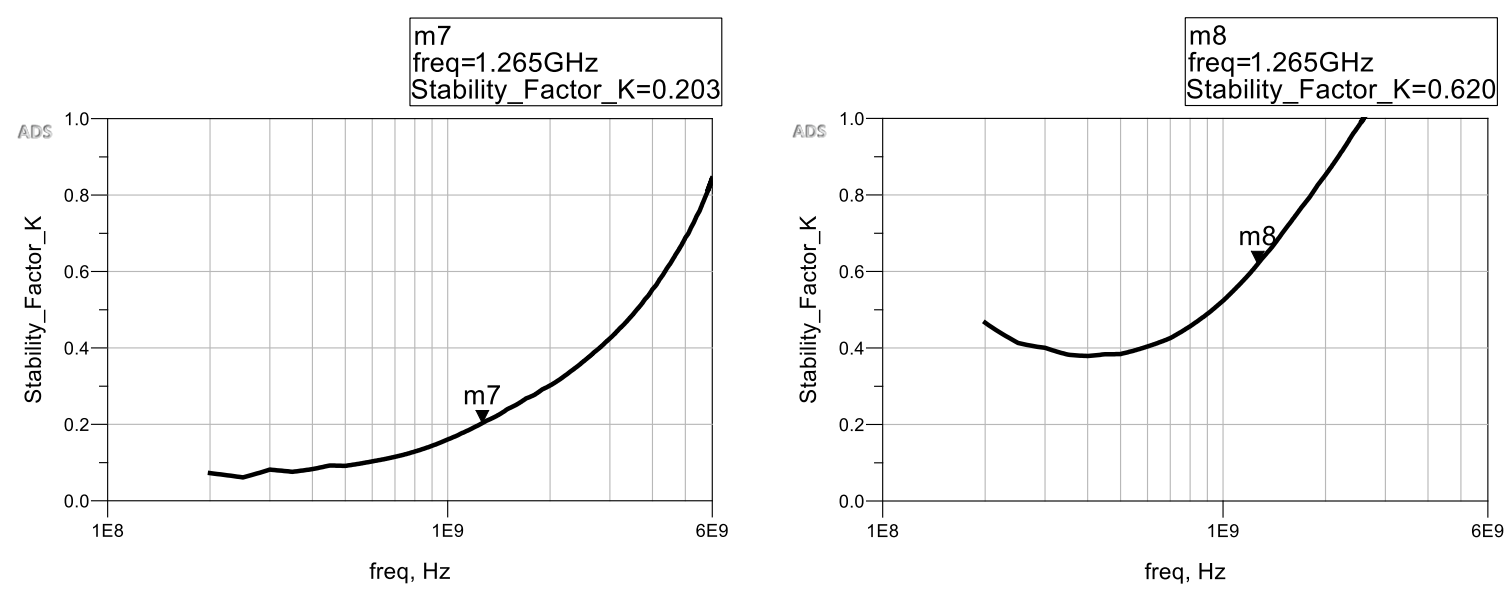

Figure 3.15: Stability Factor, K, plot of initial BFP74OF design before(left) and after (right) the addition of the stability improvements.

The next design step was to add and an input/source matching network. The goal was to add a network which increased overall transducer gain but prioritized an overall input impedance of low noise figure. A constant noise figure circle was plotted on the optimal source impedance Smith chart to help determine the source matching network impedance for minimum noise figure, shown below in Figure 3.16. It can be observed from the Smith chart below that a small positive reactance (series inductance) 
is desirable to increase the overall transducer gain while preserving a noise figure (NF) of $0.6 \mathrm{~dB}$, with a target pointed out by the arrow.

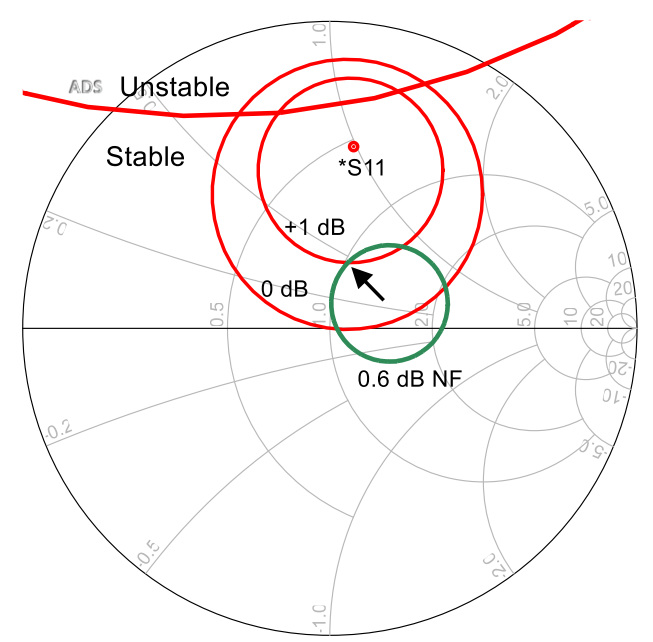

Figure 3.16: Smith chart of optimal source impedance with constant gain circles, noise figure circle, and stability circle evaluated at $1265 \mathrm{MHz}$ for initial BFP740F conditionally stable design.

The below, left, Smith chart in Figure 3.17 shows the impedance shift of a series 3.3 $\mathrm{nH}$ inductor. However, the source matching network has to provide DC current to the transistor's base terminal. An RF choke and a DC blocking capacitor are required. A shunt inductor with a large enough impedance was chosen for an RF choke which allows DC current, and a series capacitor with a small enough impedance was chosen for a DC blocking capacitor. The below, right, Smith chart in Figure 3.17 shows the measured completed source matching network, where source impedance is measured from looking backwards into the network, shown in Figure 3.18. 

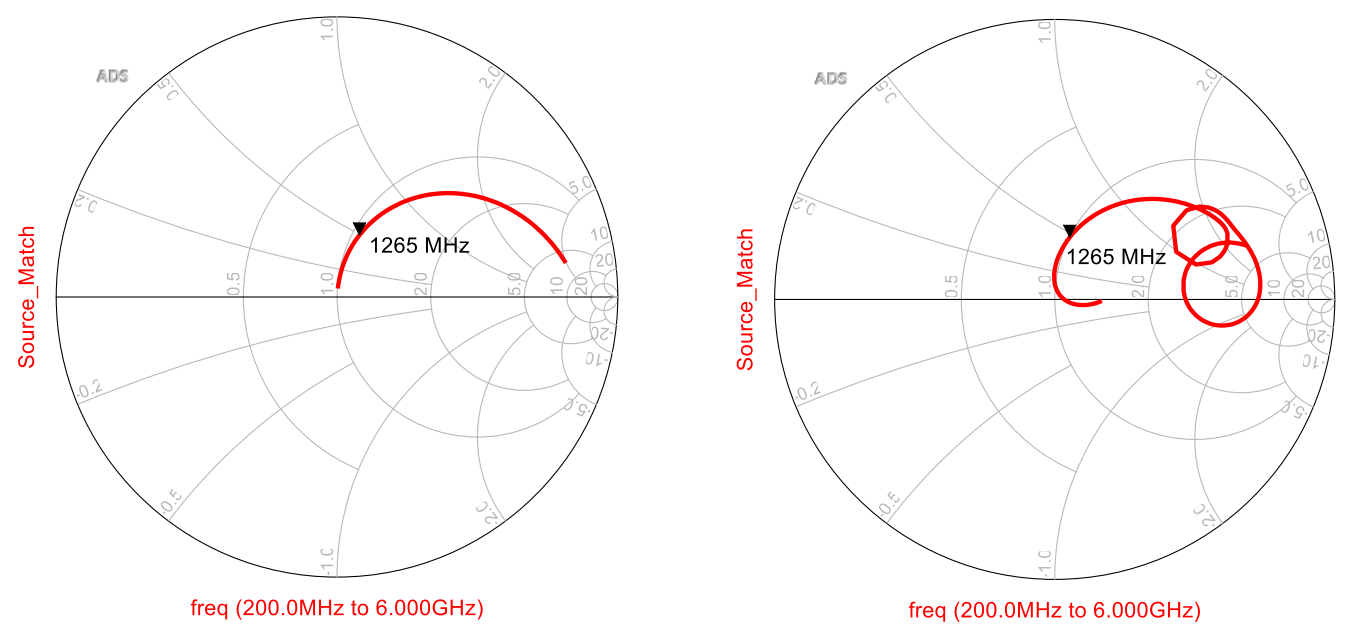

Figure 3.17: Smith charts of source impedance matching network. Series inductor only (left), and complete matching network with biasing components (right).

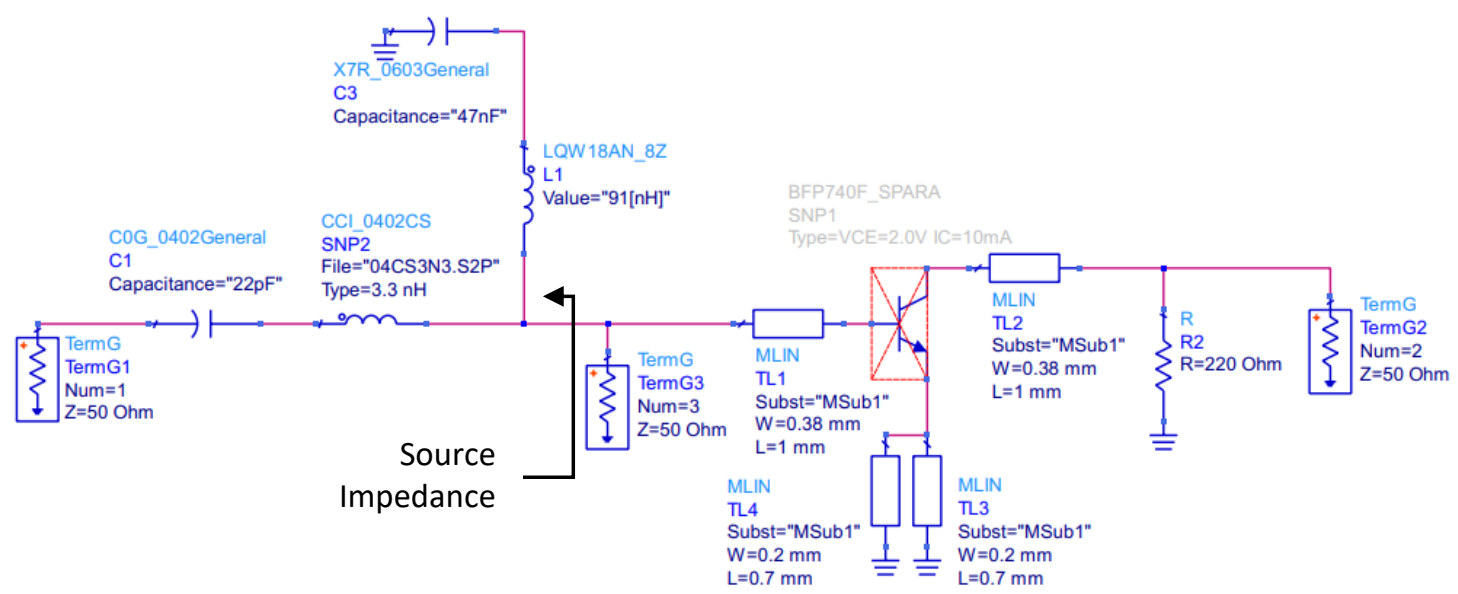

Figure 3.18: ADS schematic of stability improved BFP74OF with source matching network added, and configured for source impedance analysis.

With the source matching network designed, the output reflection coefficient $S_{22}$ can be reanalyzed. Figure 3.19 and Figure 3.20, below, show the updated Smith charts and S parameter magnitude plots. From the optimal load impedance Smith chart it can be observed that positive reactance (series inductor) is desired again, however, the first component of the output matching network has to be a shunt inductor (positive admittance) to allow DC current into the collector terminal. A large shunt inductor, large relative impedance, could be chosen to act as an RF choke at $1265 \mathrm{MHz}$ to have 
minimal effect on impedance transformation. Instead, a moderate size shunt inductor was chosen to cause a moderate positive admittance shift on the Smith chart. The moderate size shunt inductance, moderate size impedance at $1265 \mathrm{MHz}$, has a lower relative impedance at frequencies below $1265 \mathrm{MHz}$ which helps increase the stability factor below $1265 \mathrm{MHz}$.
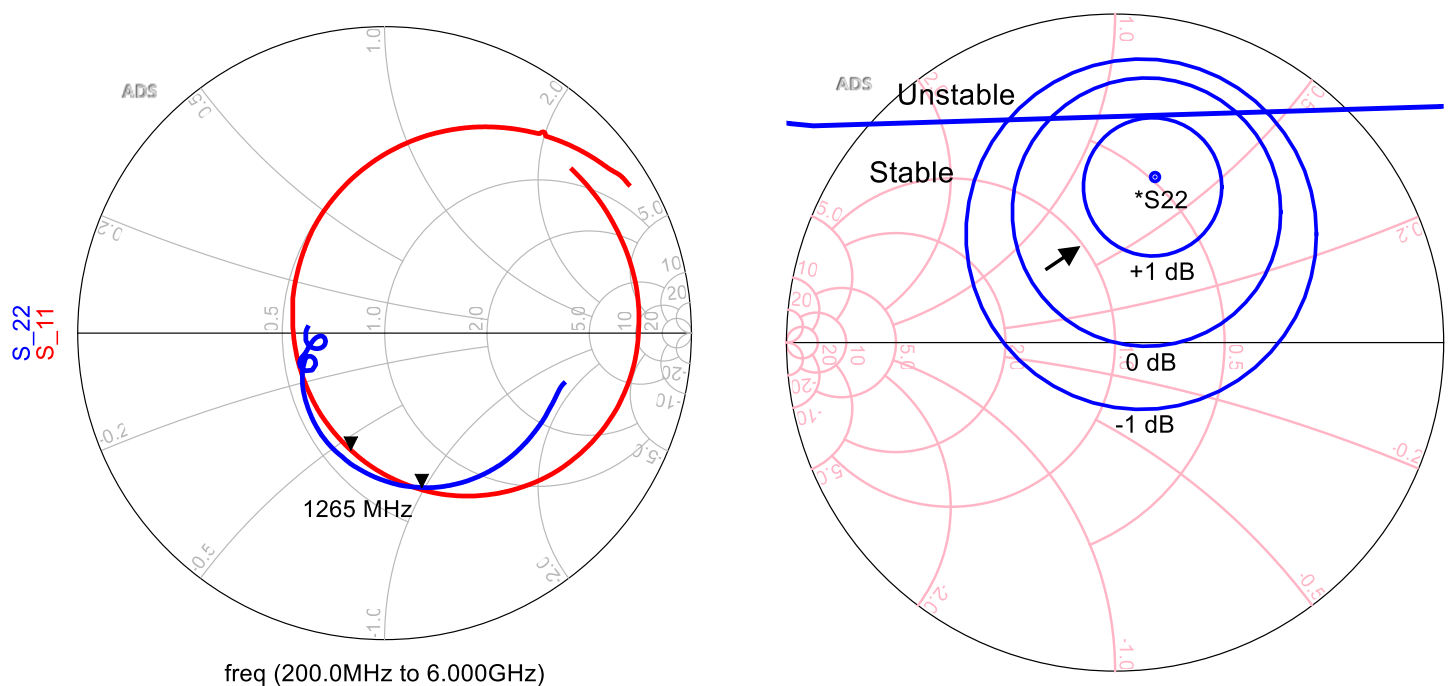

Figure 3.19: Smith chart of BFP74OF with input matching network and stability improvements (left). Smith chart of optimal load impedance, constant gain circles, and stability circle evaluated at $1265 \mathrm{MHz}$ (right).

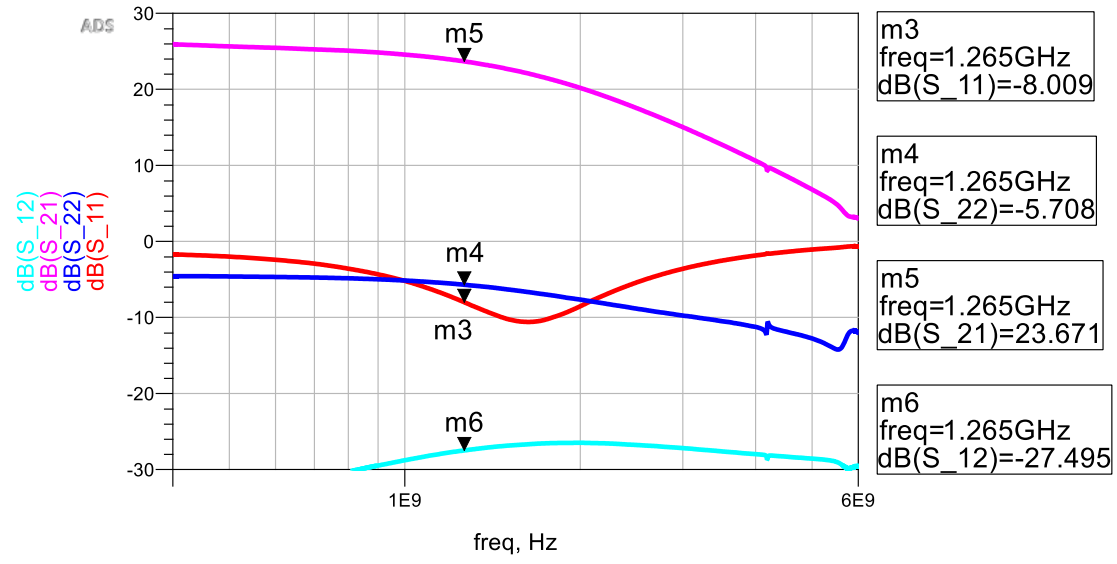

Figure 3.20: S parameter magnitude plot of BFP74OF with input matching network and stability improvements. 
The below, left, Smith chart in Figure 3.22 shows the impedance shift of a shunt $10 \mathrm{nH}$ inductor. However, the load/output matching network also has to provide DC current to the transistor's collector terminal. A biasing resistor is placed in series with the $10 \mathrm{nH}$ inductor and a DC blocking capacitor is also required. The biasing resistor diminishes the positive admittance shift of the shunt inductor which could be negated by shunt capacitance on the opposite side of the shunt inductor. However, through an iterative process it was found the design performed better by placing the shunt capacitance after the biasing resistor. This is shown in the below schematic, Figure 3.21, where load impedance is measured from looking forwards into the network.

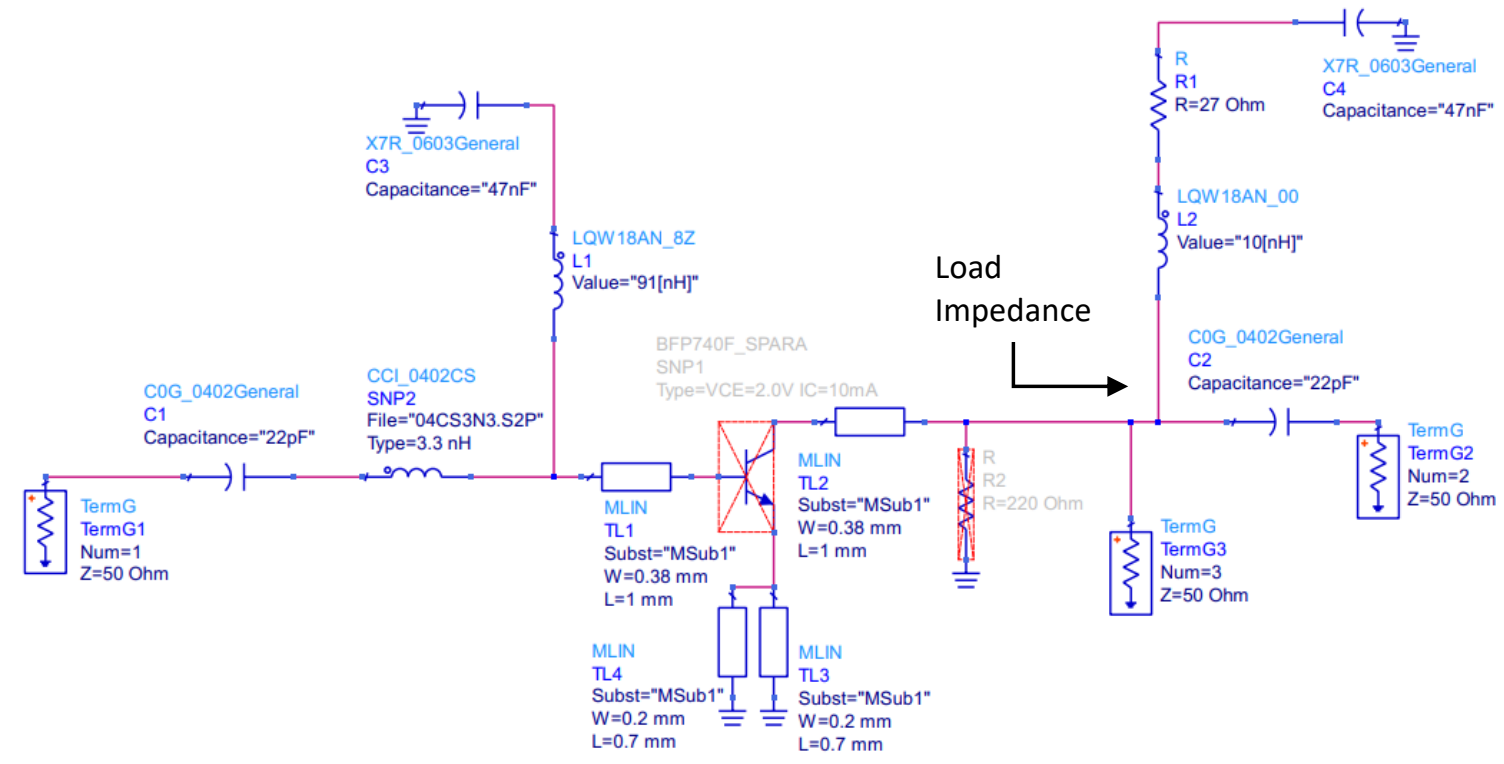

Figure 3.21: ADS schematic of stability improved BFP74OF with source and load matching network added, and configured for load impedance analysis.

The Smith charts below in Figure 3.22 show the single shunt inductor compared to the completed load matching network. 


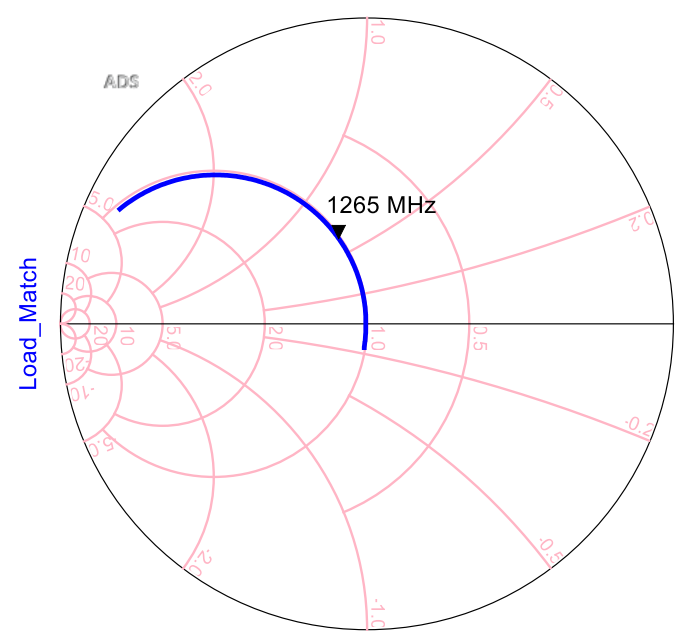

freq $(200.0 \mathrm{MHz}$ to $6.000 \mathrm{GHz})$

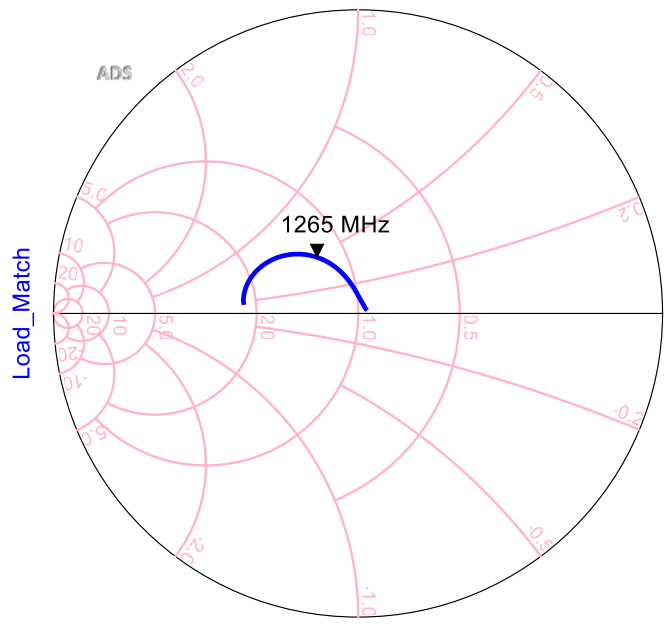

freq $(200.0 \mathrm{MHz}$ to $6.000 \mathrm{GHz})$

Figure 3.22: Smith charts of load impedance matching network. Shunt inductor only (left), and complete matching network with biasing components (right).

With the source and load matching networks completed, the design requires one more modification to the output section. The shunt stability resistor connected to the transistor's collector terminal has to be relocated to the opposite side of the DC blocking capacitor to prevent the transistor's biasing from being affected. Due to the DC blocking capacitor having negligible effect on the output matching network, the relocation of the output shunt stability resistor also has minimal effect, shown below in Figure 3.23. 


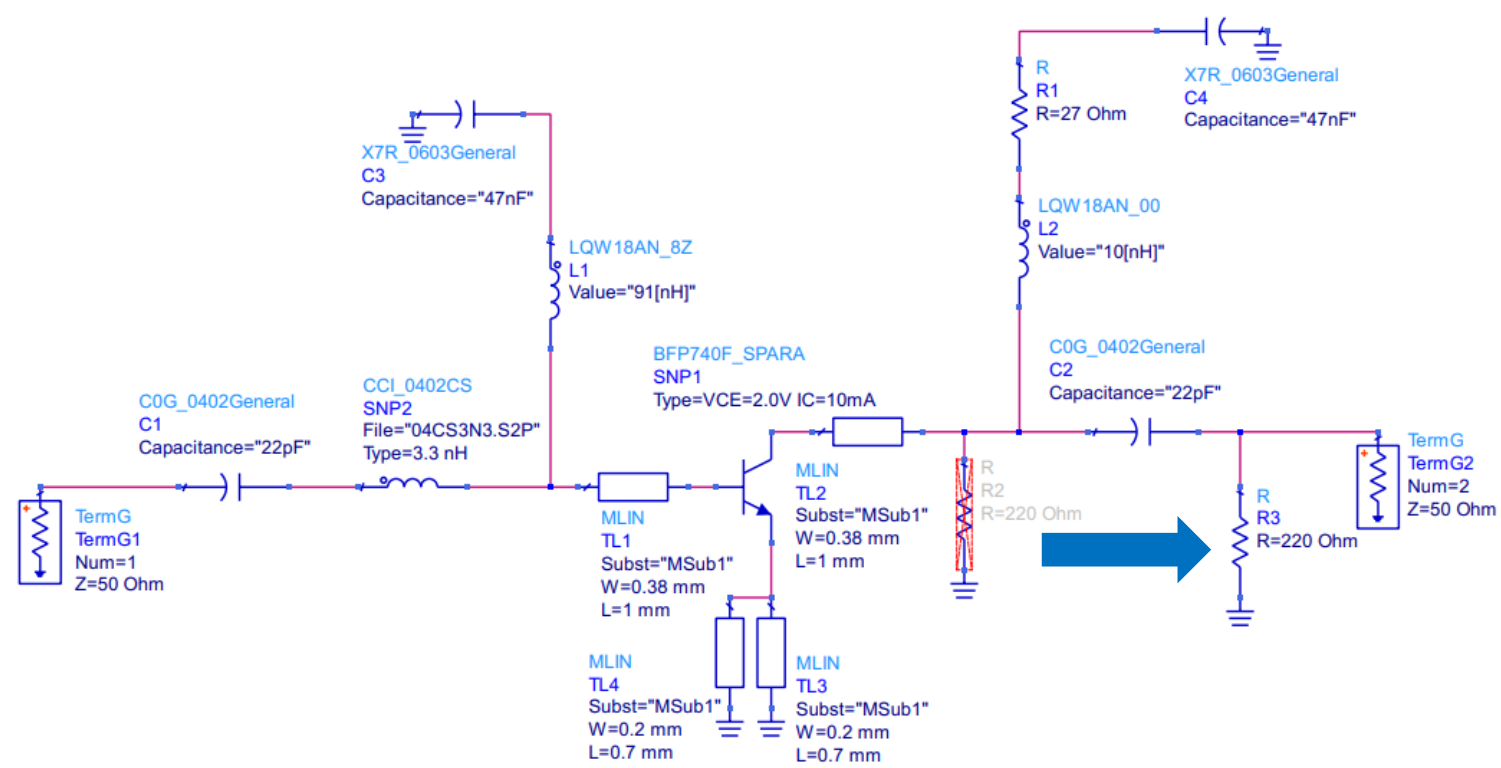

Figure 3.23: ADS schematic of BFP74OF showing updated placement of output shunt stability resistor.

The final L-band LNA design with the Infineon BFP740F RF BJT transistor is shown in the schematic below, Figure 3.24. The input series inductor value was changed from 3.3 $\mathrm{nH}$ to $2.0 \mathrm{nH}$ due to available inventory which also minimally affected the design.

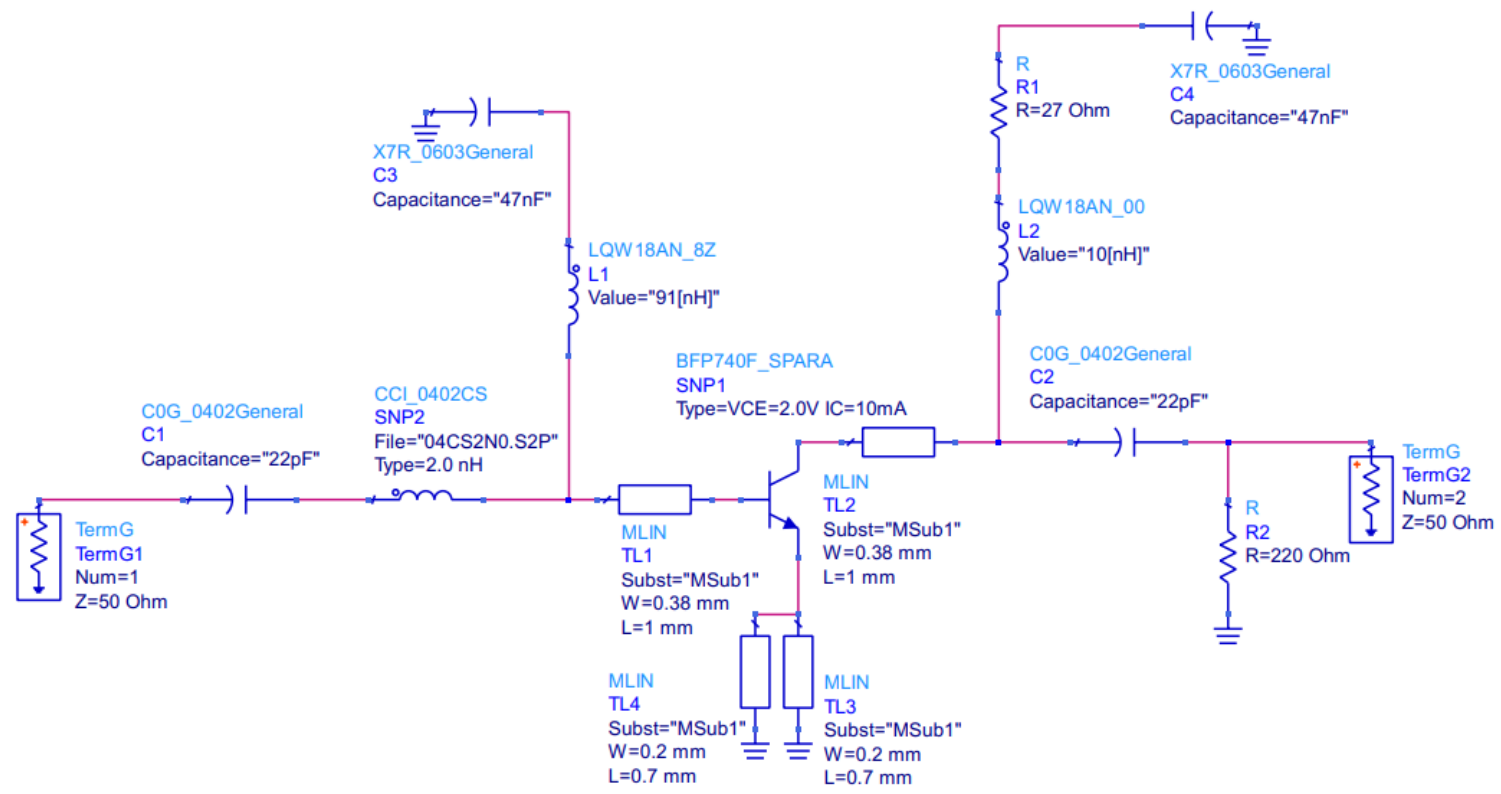

Figure 3.24: Final L-band LNA design with Infineon BFP74OF RF BJT 

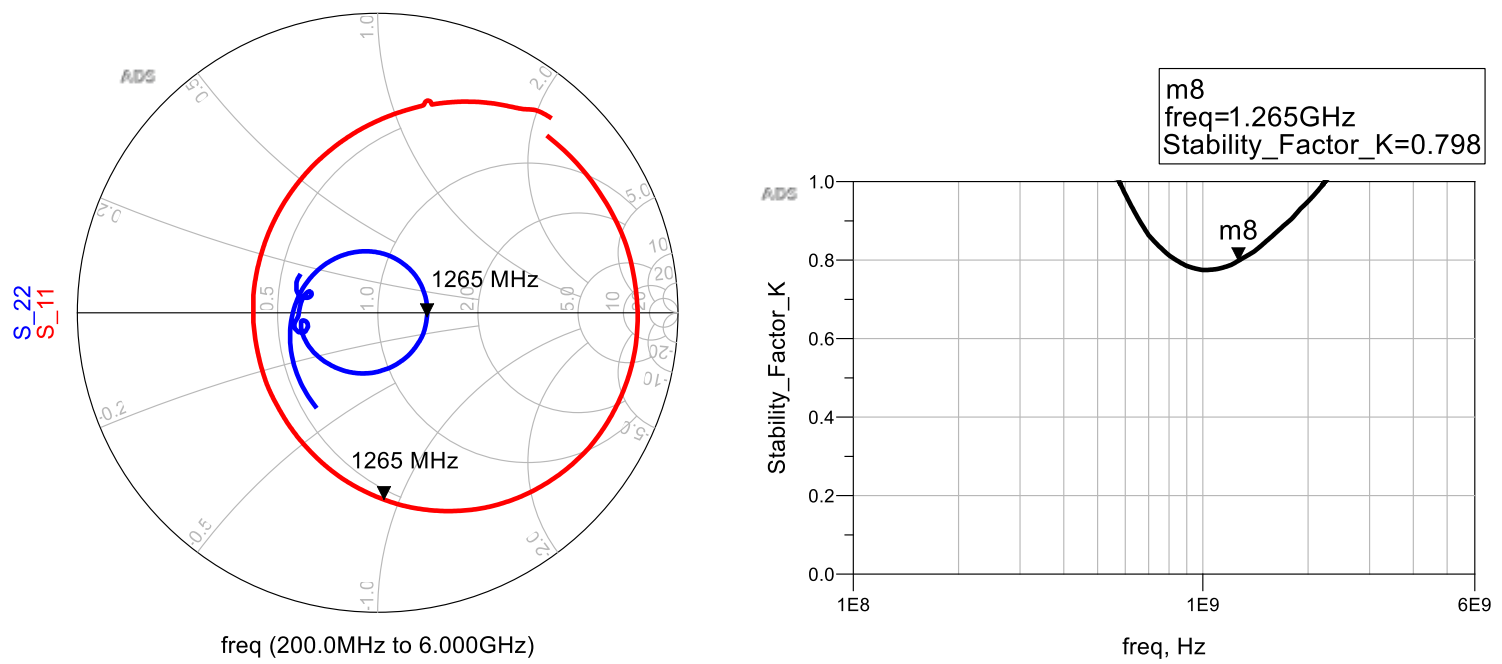

Figure 3.25: Smith chart of BFP74OF final design (left) with stability factor plot (right)
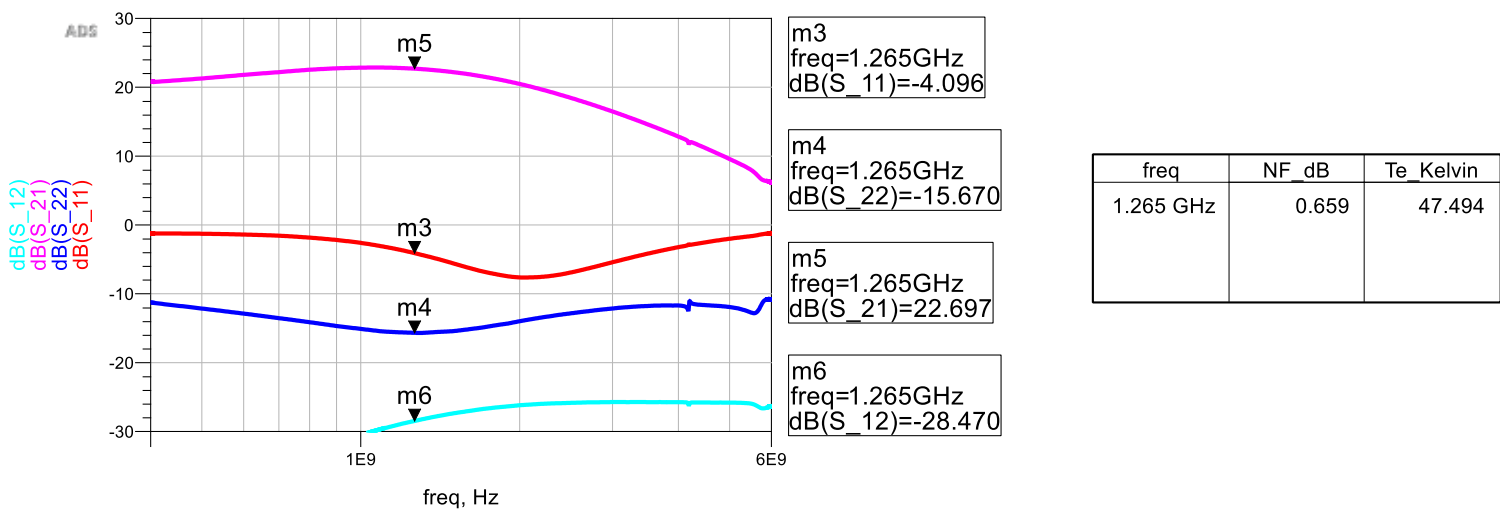

Figure 3.26: S parameter magnitude plot of BFP74OF BJT final design

The final S parameters and stability factor results are shown above in Figure 3.25

and Figure 3.26. The figure above also shows the simulated noise figure (NF) and corresponding effective noise temperature. The simulated design goal was reached with an effective noise temperature of $47.5 \mathrm{~K}, 0.66 \mathrm{~dB}$ NF. The design is still conditionally stable, however, after adding input and output filtering it becomes unconditionally stable. This will be shown later in section [4.1.1 L-Band S Parameters]. The final schematic including all biasing components are shown below in Figure 3.27. 


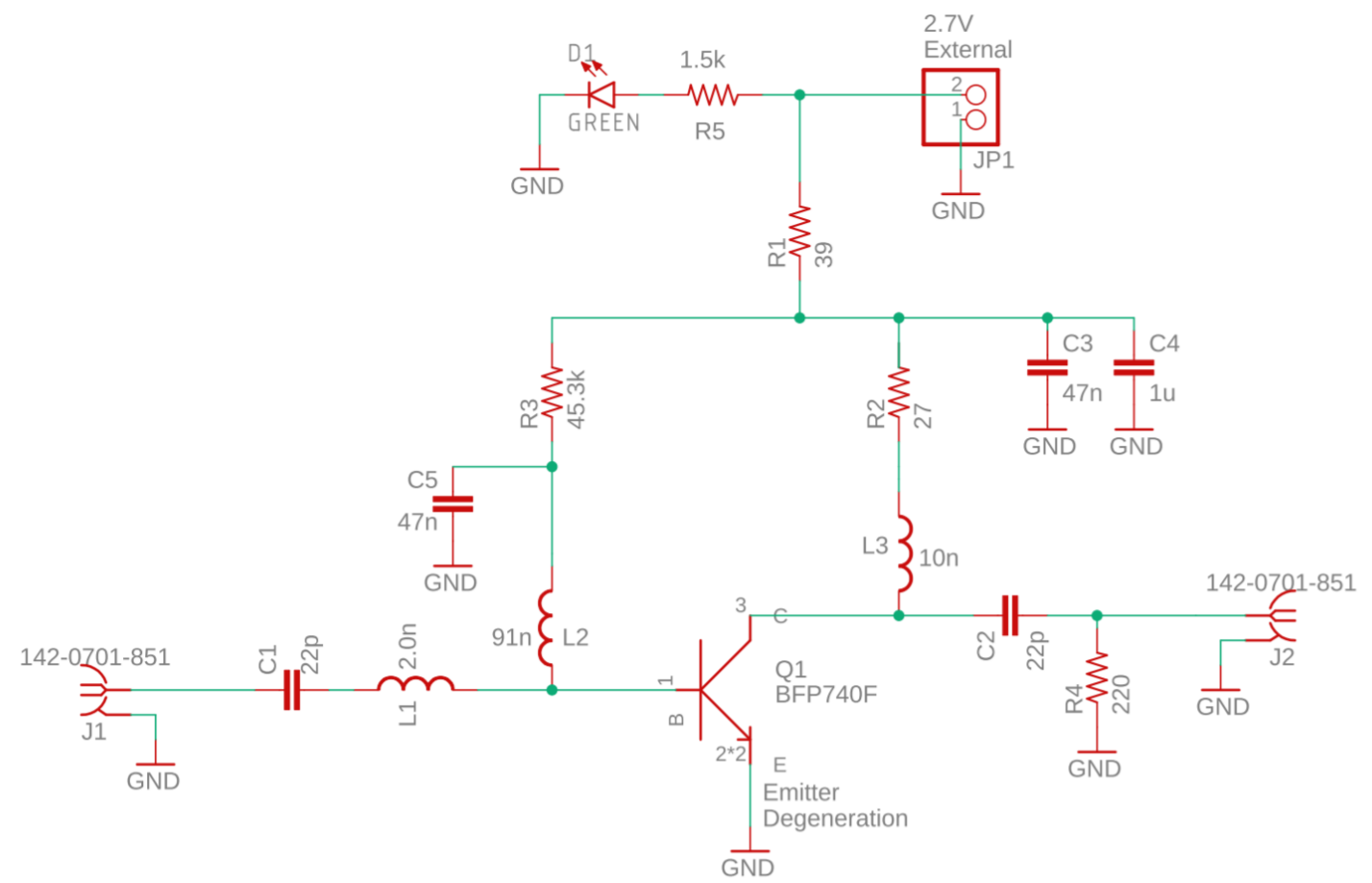

Figure 3.27: Final EAGLE CAD schematic of L-band LNA with Infineon BFP74OF RF BJT

\subsection{Transmitter}

To validate a communication system link budget a receiver and transmitter are required. The official OreSat1 CubeSat ground station L-band transmitter design was incomplete; therefore, a simple transmitter utilizing the same radio IC and frequency conversion components as the receiver was designed for the purpose of link analysis. The official ground station will utilize a LimeSDR, a software-defined radio (SDR), from Lime Microsystems.

The transmitter design, shown below in Figure 3.28, is a reverse version of the superheterodyne receiver architecture. It employs an upconverter for frequency conversion. The receiver IC used in the receiver design is actually a transceiver IC which can receive and transmit. A transmit IF of $505 \mathrm{MHz}$ was chosen with an LO frequency of 
$760 \mathrm{MHz}$ to produce $1265 \mathrm{MHz}$. This combination of IF and LO were chosen in combination with the hairpin filter characteristics to suppress intermodulation created from the mixer as much as possible. A hairpin filter is a type of distributed-element filter which looks and acts similar to an acoustic tuning fork, shown below in Figure 3.30.

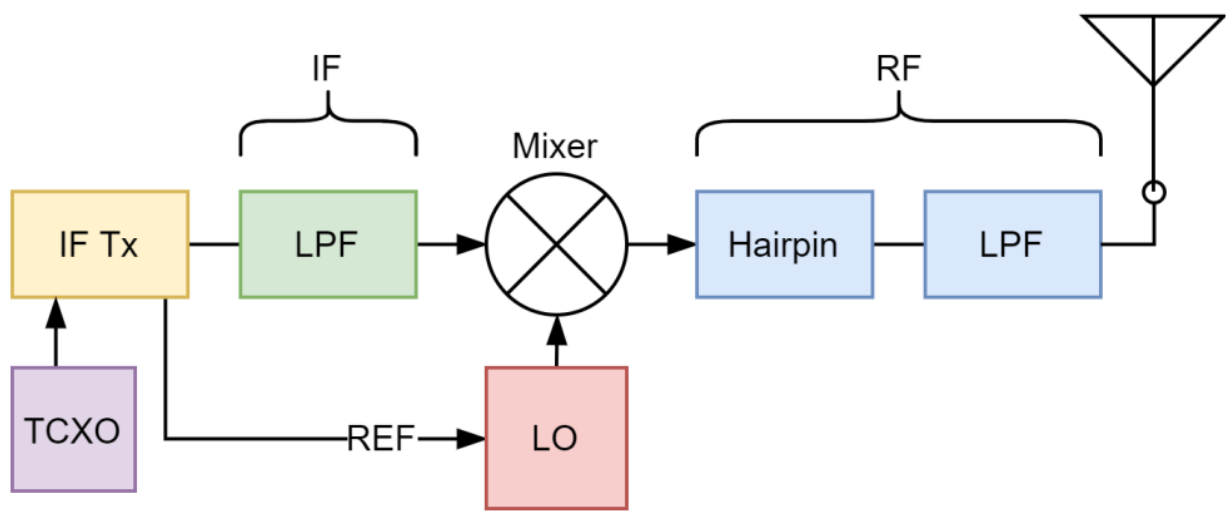

Figure 3.28: L-band Prototype block diagram

The spurious emissions from intermodulation are shown in Figure 3.29. The highest measured spurious emission was $-53 \mathrm{dBc}$ (decibels relative to carrier power). The transmitter was constructed using the same methods as the receiver, and the completed prototype L-band transmitter is shown below in Figure 3.31.

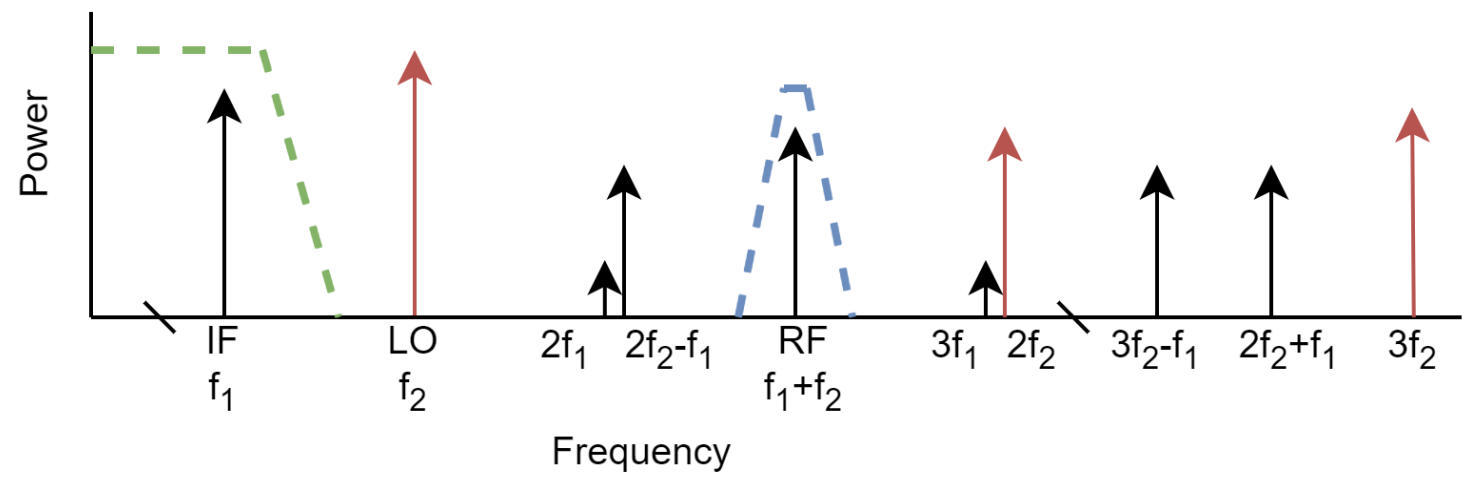

Figure 3.29: RF Spectrum of frequency up conversion 


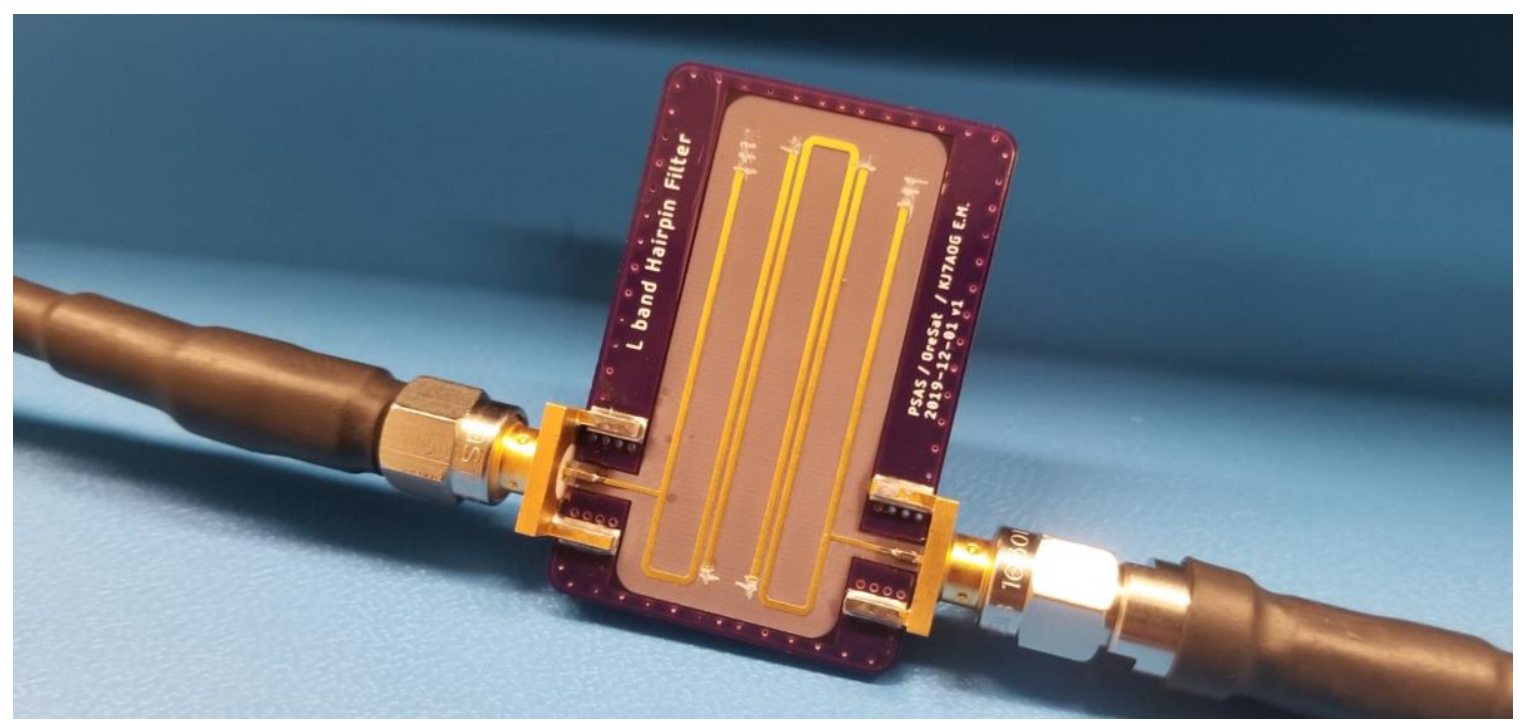

Figure 3.30: L-band (1265 MHz) hairpin filter designed by author

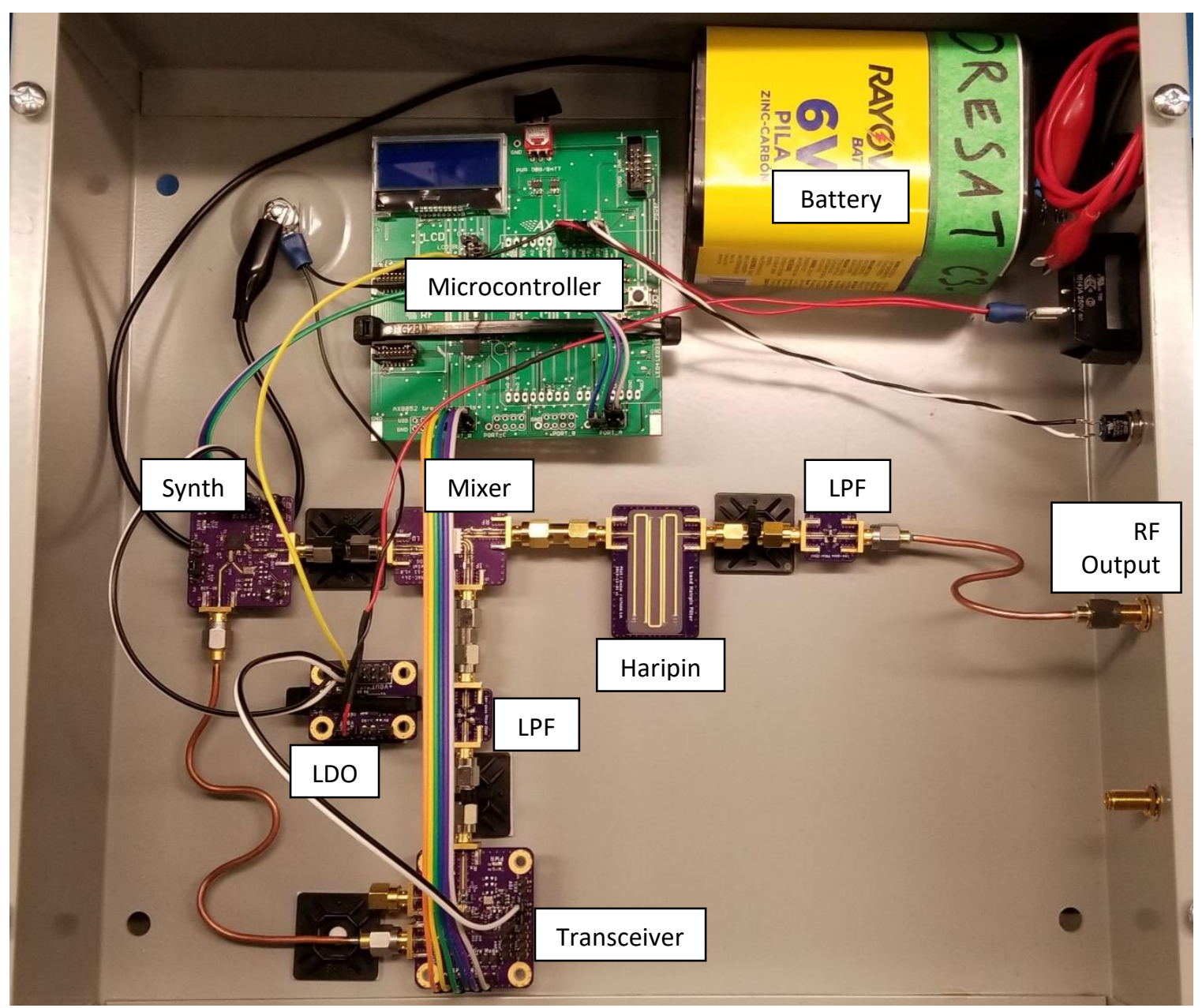

Figure 3.31: Prototype L-band transmitter (purple boards) in steel box. Designed and assembled by author. 


\subsection{Transceiver IC and Microcontroller}

The L-band receiver and transmitter uses the AX5043 transceiver IC from ON Semiconductor [13]. A microcontroller communicates with the AX5043 via SPI (Serial Peripheral Interface) and an interrupt line. The CubeSat, OreSat1, will incorporate the ST Microelectronics STM32F446RE microcontroller which includes an Arm Cortex-M4 32-bit RISC core. However, for the purpose of link budget validation the DVK-BASE-2GEVK development kit by ON Semiconductor was used due to the incorporation of biterror-rate test (BERT) functionality [14]. The evaluation board uses an AX8052F100 microcontroller and is programmed with a development software suite from ON Semiconductor called AX-RadioLAB [15]. In addition, custom code was developed for programming the Silicon Labs Si4112 synthesizer using a proprietary 3-wire serial interface [16].

\section{Measurements and Results}

The first section of this chapter will discuss the preliminary hardware measurements for the L-band receiver including S parameters, conversion gain, spurious emissions, and noise figure. The following section will discuss receiver validation method and results. Finally, in the last section the results discovered during receiver validation are used to reanalyze the CubeSat link budget. 


\subsection{Hardware Characterization}

\subsubsection{L-Band S Parameters}

In section [3.1.4 First Stage LNA Design], the receiver's initial low noise amplifier (LNA) was designed for close to minimum noise figure while considering maximum gain for a reasonable conditional stability. The design required only $10 \mathrm{~mA}$ at $2.7 \mathrm{~V}$ with a measured output $1 \mathrm{~dB}$ compression point (OP1dB) of $-2.5 \mathrm{dBm}$. Figure 4.1 and Figure 4.2 show the measured results of the Infineon BFP740F L-band LNA prototype board imported into ADS. S parameter characterization was measured on an HP 8753E vector network analyzer (VNA).
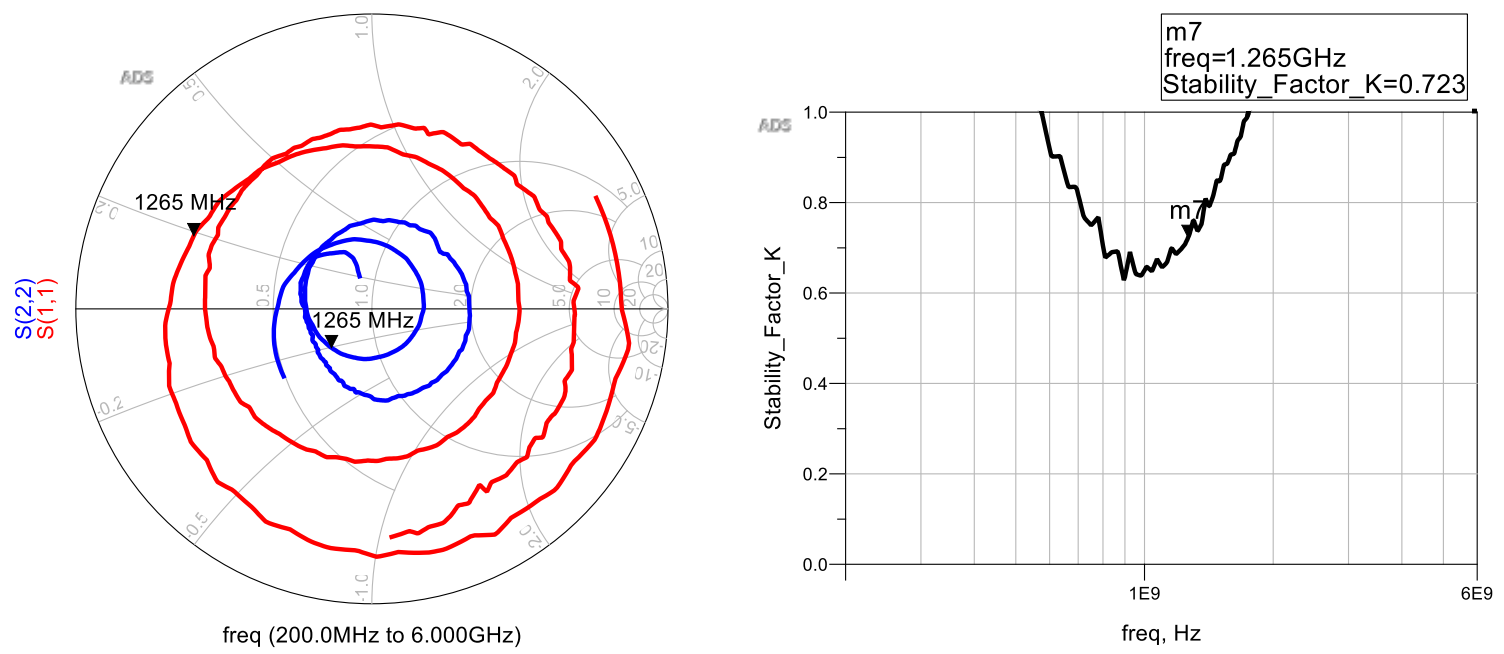

Figure 4.1: Smith chart (left) and stability factor (right) of measured BFP74OF L-band LNA prototype 


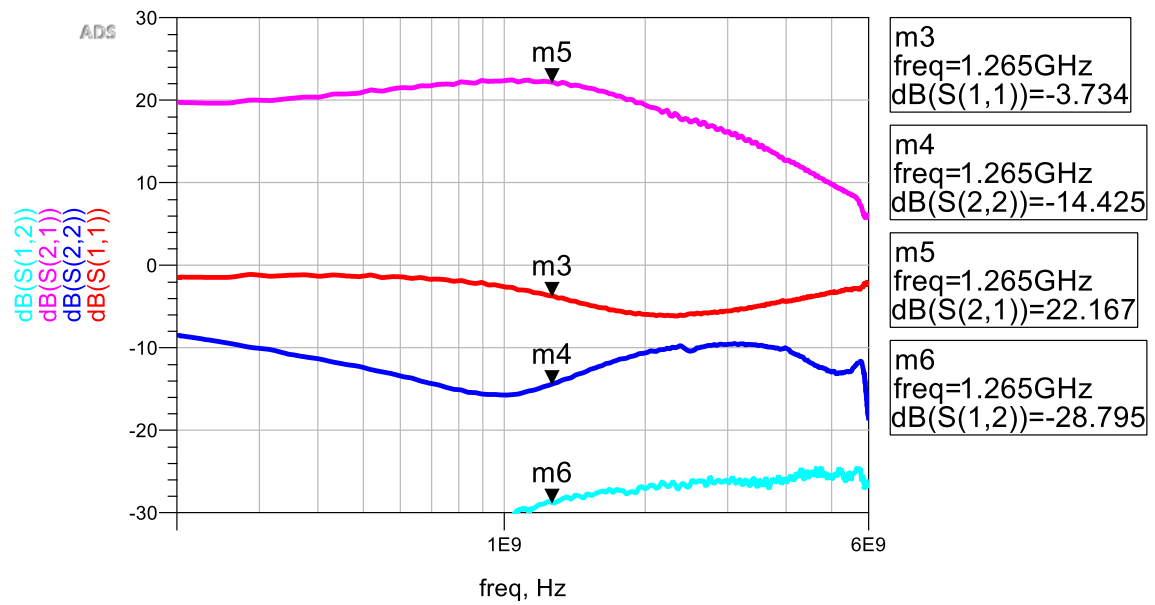

Figure 4.2: Magnitude plot of the measured BFP74OF L-band LNA prototype

The LNA was then placed in the L-band receiver RF chain which includes bandpass filters (BPFs), a GPS diplexer, and the second stage LNA. The second stage LNA is another prototype design using an Infineon BGB741L7ESD monolithic microwave integrated circuit (MMIC) which consumes only $5.5 \mathrm{~mA}$ at $2.7 \mathrm{~V}$ and provides a measured OP1dB of over $+10 \mathrm{dBm}$. It is important to note the OP1dB for both LNAs are much higher than required for this L-band receiver as it will never receive greater than -90 $\mathrm{dBm}$ of carrier power at its input terminal. Figure 4.3 below, shows the entire RF (1265 $\mathrm{MHz}$ ) portion of the L-band receiver's front-end with AVX BP0805A1308 thin-film BPFs. 


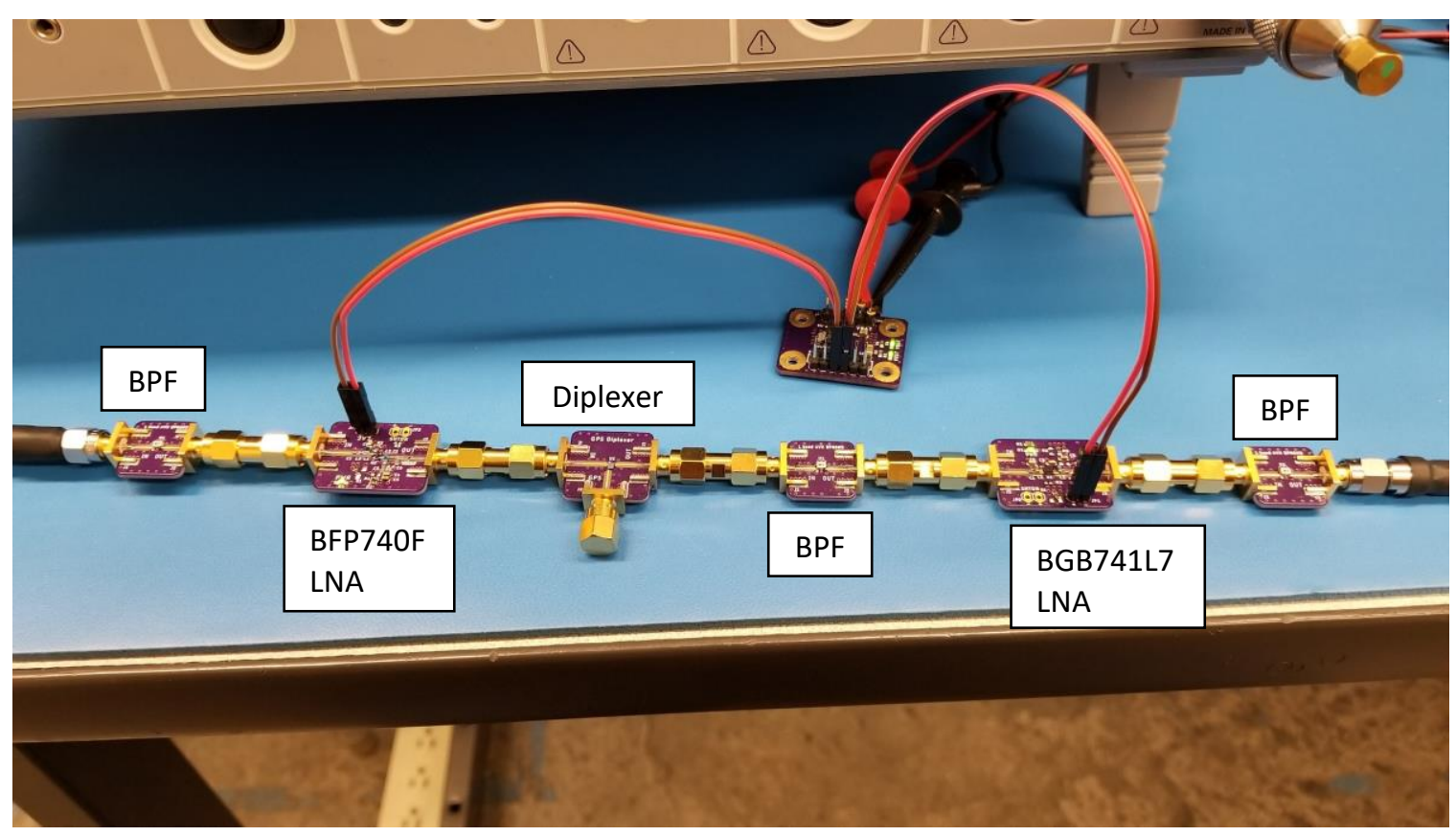

Figure 4.3: L-band receiver RF portion front-end components in VNA measurement

Figure 4.4 shows the measured results of the entire L-band front-end RF portion. The RF chain as whole becomes unconditionally stable as observed in the stability factor plot (right) in Figure 4.4. The RF chain has a total transducer gain of $35.8 \mathrm{~dB}$ and a minimum stability factor value of greater than one.
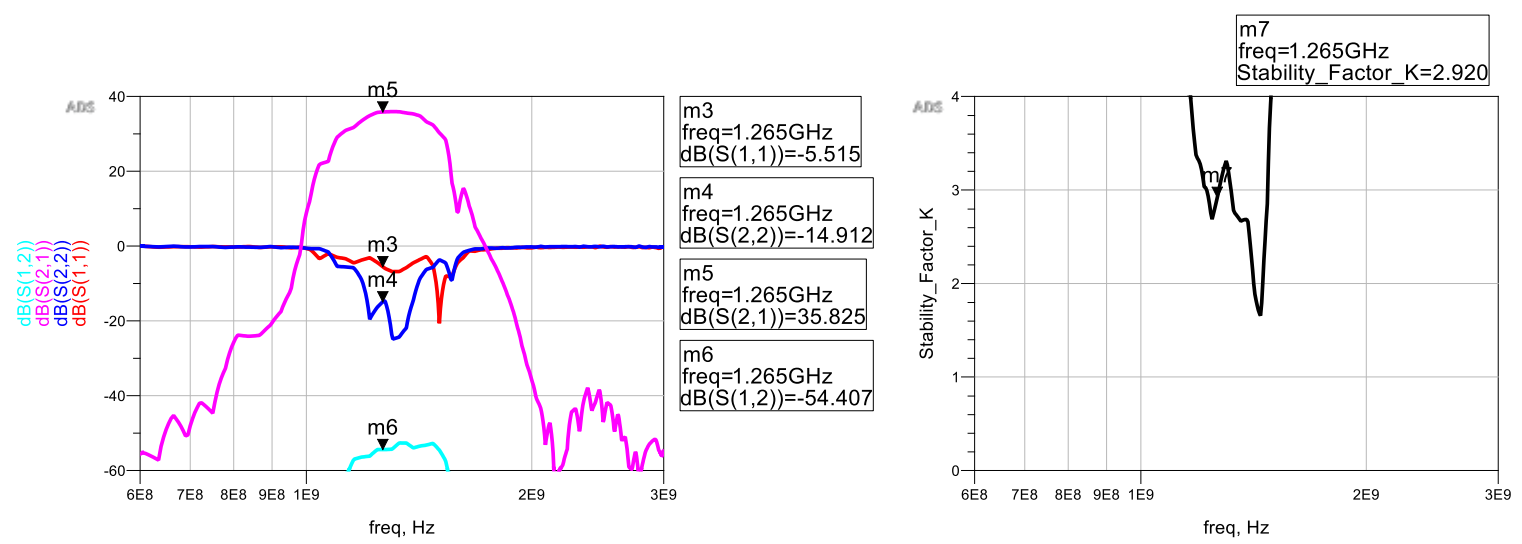

Figure 4.4: S parameter magnitude plot (left) and stability factor (right) of measured L-band RF front-end chain. 


\subsubsection{Frequency Conversion}

A significant amount of effort was contributed to maximizing efficiency of the mixer's conversion loss. The Mini-Circuits MAC-24+ diode ring balanced mixer was chosen for frequency conversion which required a suggested +4 to $+10 \mathrm{dBm}$ local oscillator power. Initially a pre-amplifier was designed to be used after the synthesizer, however, after designing simple matching networks for the mixer's RF and IF ports the pre-amp was not required. A conversion loss of $10 \mathrm{~dB}$ was measured for the mixer without the LO pre-amp and $6 \mathrm{~dB}$ with the pre-amp. In addition, the pre-amp required significantly more power for the receiver due to providing $+10 \mathrm{dBm}, 18 \mathrm{~mA}$ at $2.7 \mathrm{~V}$. If the $4 \mathrm{~dB}$ of extra loss without the pre-amp was a concern than an additional LNA in the IF path could be added to achieve more gain with less current, $6 \mathrm{~mA} @ 2.7 \mathrm{~V}$ with the same LNA. Figure 4.5 shows the entire L-band front-end with frequency conversion being evaluated with $1.265 \mathrm{GHz}$ being injected into the RF input and $457 \mathrm{MHz}$ being measured from the IF output with an $808 \mathrm{MHz}$ LO at $-2.8 \mathrm{dBm}$. Conversion gain of the entire front-end measured at $+25.9 \mathrm{dBm}$ excluding cable losses. 


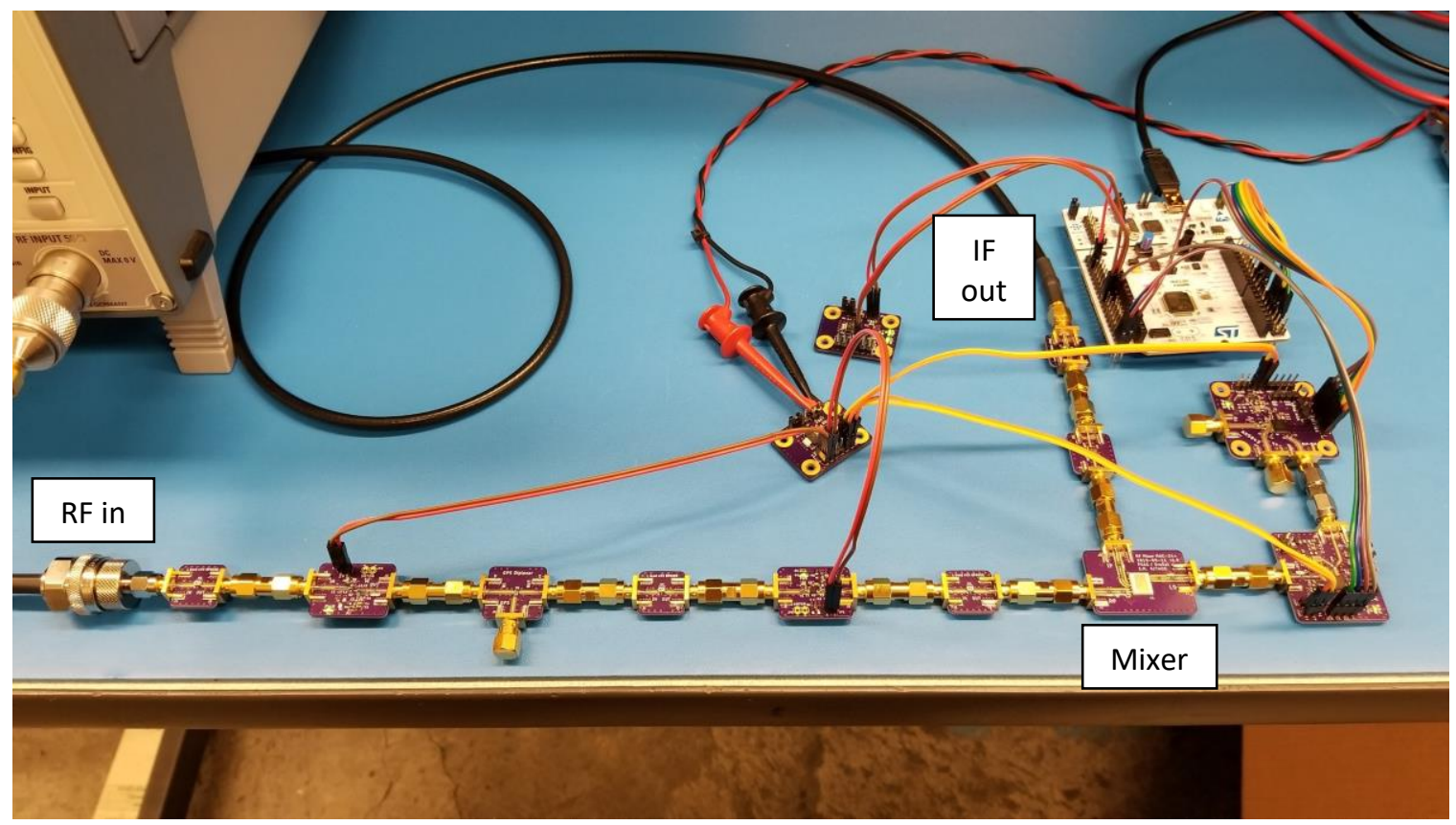

Figure 4.5: L-band receiver front-end in a conversion gain measurement

\subsubsection{Spurious Emissions}

Spurious emissions from the input of a superheterodyne receiver may be a concern if the local oscillator (LO) is providing too much power to the mixer and/or there is not enough reverse isolation of the RF path. The emissions will radiate from the receiver's antenna causing potential electromagnetic interference (EMI) to the satellite itself or nearby spacecraft. Local oscillator leakage from the mixer in this receiver are already reduced due to excluding the pre-amplifier from the LO. Reverse isolation is also high due to the two LNA stages in the RF path which provide $50 \mathrm{~dB}$ combined over a wide frequency range. The spurious emissions caused by the LO and non-linear effects of the mixer are summarized below in Table 4.1. 


\begin{tabular}{c|c} 
Frequency $(\mathrm{MHz})$ & Power $(\mathrm{dBm})$ \\
\hline $808 \mathrm{MHz}$ & -98 \\
$1616 \mathrm{MHz}$ & -120 \\
$2424 \mathrm{MHz}$ & -101 \\
$3232 \mathrm{MHz}$ & -115 \\
$4848 \mathrm{MHz}$ & -117
\end{tabular}

Table 4.1: L-band receiver input port spurious emissions

\subsubsection{Noise Figure}

Noise figure (NF) measurements were taken for all front-end components using the HP $8970 \mathrm{~B}$ noise figure meter along with the Agilent 346A noise source. A wide-band LNA prototype was designed and used as a pre-amplifier to reduce the effective noise figure input of the measurement system, with a NF of $1.0 \mathrm{~dB}$ at L-band and $1.1 \mathrm{~dB}$ at UHF. All passive devices measured as expected and the mixer measured about $7 \mathrm{~dB} N \mathrm{~F}$ for $1.265 \mathrm{GHz}$ to $457 \mathrm{MHz}$ including IF filters (SAW and LPF) for a single side-band down conversion measurement. The first and second stage LNAs used in the L-band receiver measured as expected at $0.66 \mathrm{~dB}$ NF and $1.24 \mathrm{~dB}$ NF respectively. Figure 4.6 below, shows the first stage L-band receiver LNA in a NF measurement, labeled as the device under test (DUT). 


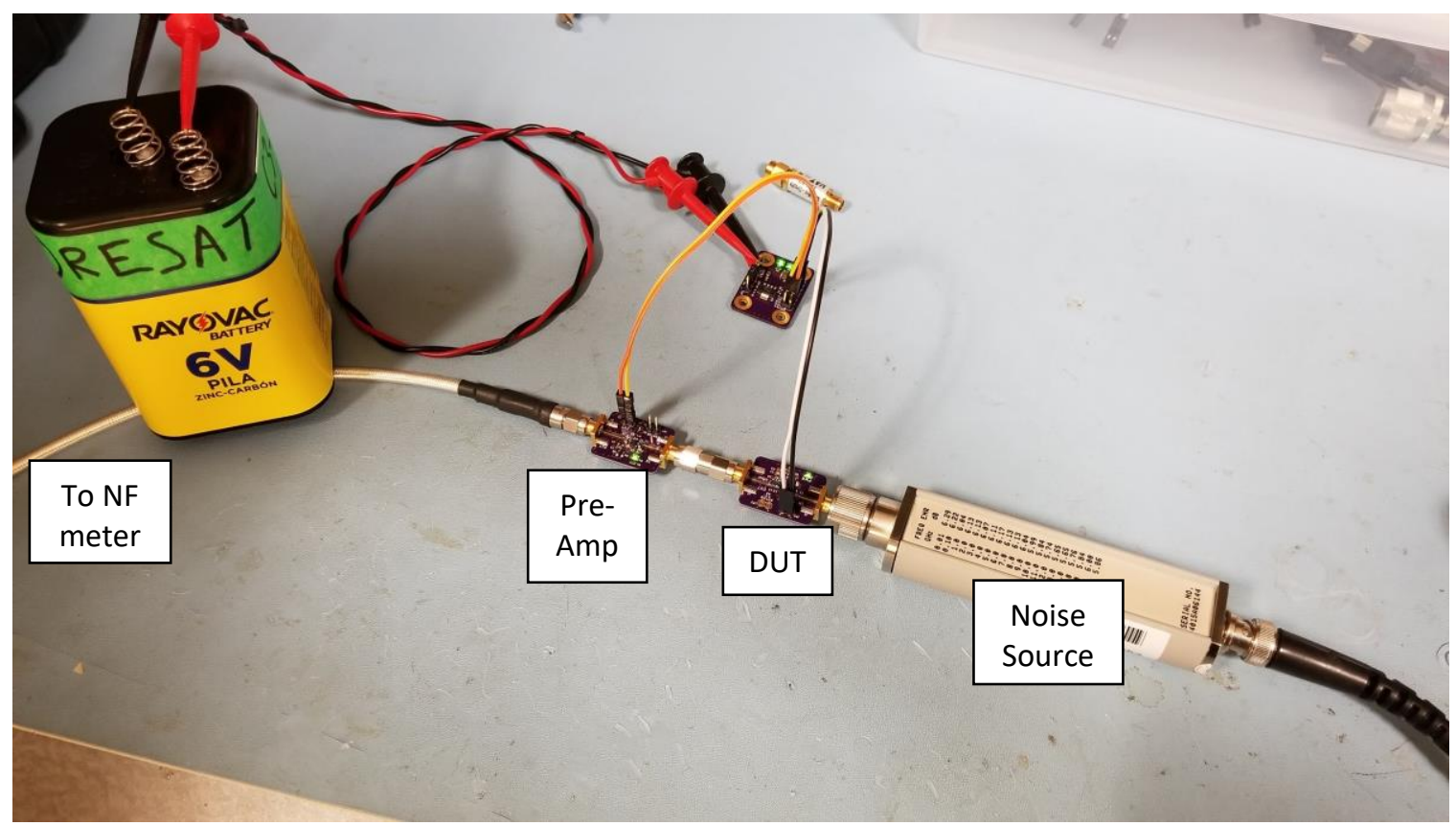

Figure 4.6: L-band first stage LNA in a NF measurement

In addition, the entire L-band receiver front-end was measured in a down conversion measurement shown below in Figure 4.7. A $10 \mathrm{~dB}$ attenuator was added after the pre-amp to reduce the total gain of the system since it would have been close to $40 \mathrm{~dB}$ of gain. The entire L-band front-end measured $1.79 \mathrm{~dB}$ NF. This measurement was used for validating the receiver's effective system noise temperature by comparing the measured value to the calculated total. Table 4.2, below, with Friis formula for noise temperature, Equation (4.1) below, shows the cascaded noise temperature of all the L-band front-end components. In summary, the calculated total of individually measured components equaled the combined measured components. 


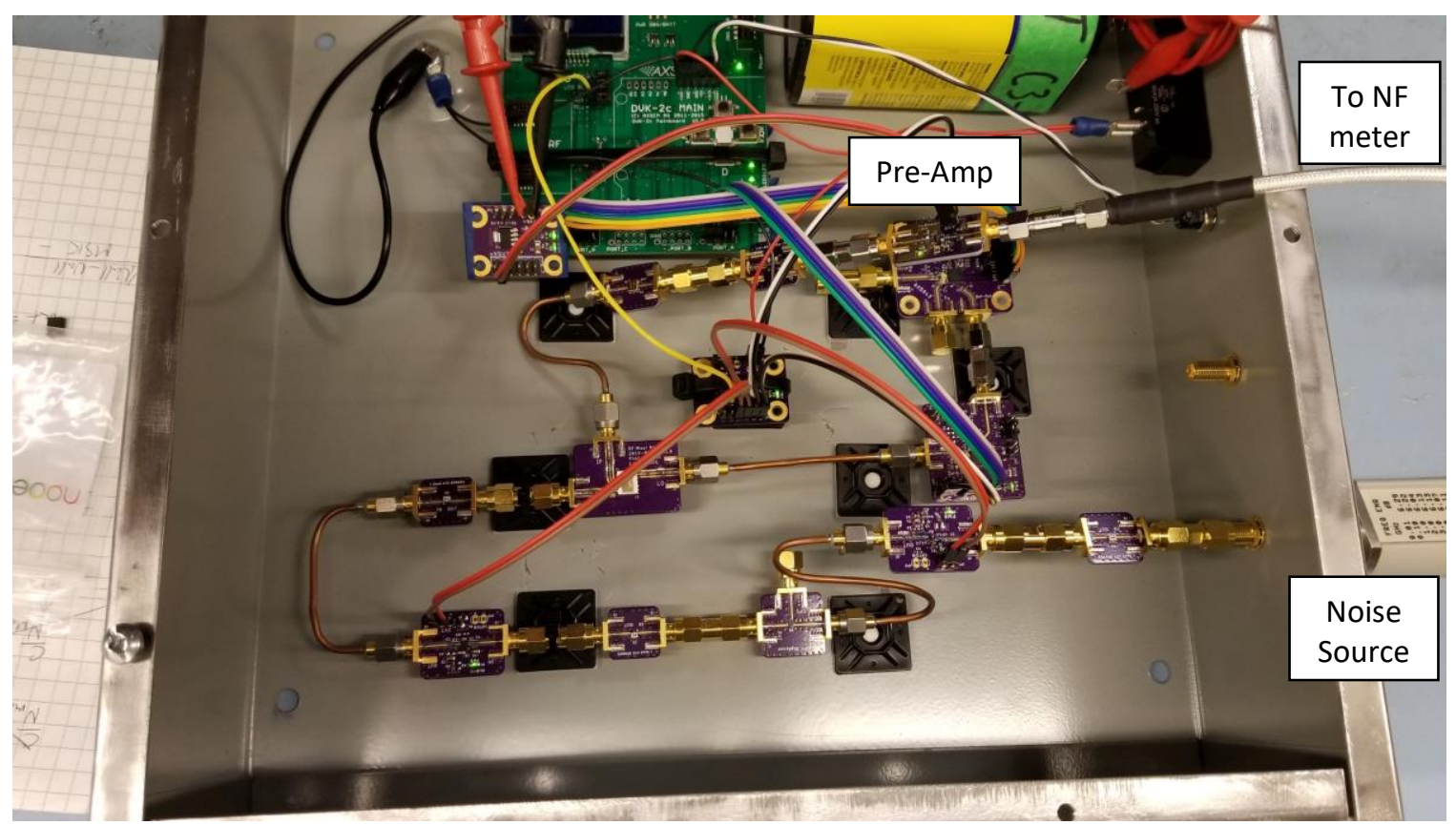

Figure 4.7: L-band prototype receiver front-end in a NF measurement

$$
T_{\text {Total }}=T_{1}+\frac{T_{2}}{G_{1}}+\frac{T_{3}}{G_{1} G_{2}}+\cdots+\frac{T_{n}}{G_{1} \cdots G_{n-1}}
$$

\begin{tabular}{c|l|ccccc|c|c}
$\#$ & Stage & $\mathrm{NF}(\mathrm{dB})$ & $\mathrm{G}(\mathrm{dB})$ & $\mathrm{T}_{\text {Stage }}(\mathrm{K})$ & $\mathrm{G}($ ratio) & ${ }^{*}$ Gains & ${ }^{*} \mathrm{G}(\mathrm{K})$ & $\mathrm{T}_{\text {Total }}(\mathrm{K})$ \\
\hline 1 & Conn. & 0.1 & -0.1 & 6.75 & 0.977 & 1 & 6.75 & 6.8 \\
2 & BPF & 1.0 & -1.0 & 75.1 & 0.794 & 0.977 & 76.84 & 83.6 \\
3 & 1st LNA & 0.66 & 22.0 & 47.6 & 158 & 0.776 & 61.32 & 144.9 \\
4 & Diplexer & 0.7 & -0.7 & 50.7 & 0.851 & 123 & 0.41 & 145.3 \\
5 & BPF & 1.0 & -1.0 & 75.1 & 0.794 & 105 & 0.72 & 146.0 \\
6 & 2nd LNA & 1.24 & 17.7 & 95.8 & 58.9 & 83.2 & 1.15 & 147.2 \\
7 & BPF & 1.0 & -1.0 & 75.1 & 0.794 & 4898 & 0.02 & 147.2 \\
8 & Mixer & 8.0 & -10.0 & 1540 & 0.100 & 3890 & 0.40 & 147.6 \\
9 & SAW & 0.9 & -0.9 & 66.8 & 0.813 & 389 & 0.17 & 147.8 \\
10 & LPF & 1.0 & - & 75.1 & - & 316 & 0.24 & 148.0 \\
\hline
\end{tabular}

Table 4.2: Noise temperature of front-end components of $L$-band receiver evaluated with Friis formula (prototype box used for validation) 
In chapter 2, section [2.3 Effective System Noise Temperature], a formula for the receiver's effective system noise temperature $\left(T_{S}\right)$ was derived. Based on the noise figure measurements taken, the effective system noise temperatures of the prototype receiver presented in chapter 3 and from the CubeSat link budget presented in chapter 2 are calculated below. Equation (4.2) shows the formula used for the prototype receiver along with $T_{R}$ calculated above in Table 4.2, and Equation (4.3) shows the formula used for the CubeSat link budget along with $T_{R}$ calculated below in Table 4.3.

$$
\begin{array}{lc}
\text { Receiver Validation: } & T_{S}=T_{\text {Input }}+T_{R} \\
\text { CubeSat Link Budget: } & T_{S}=T_{A}+\left(L_{\text {Feed }}-1\right) T_{\text {Feed.Phy }}+T_{R} L_{\text {Feed }}
\end{array}
$$

\begin{tabular}{c|lccccc|c|c}
$\#$ & Stage & $\mathrm{NF}(\mathrm{dB})$ & $\mathrm{G}(\mathrm{dB})$ & $\mathrm{T}_{\text {Stage }}(\mathrm{K})$ & $\mathrm{G}($ ratio $)$ & $*_{\text {Gains }}$ & $\mathrm{T}_{\text {Stage }} /$ & \\
\hline 1 & 1st LNA & 0.66 & 22.0 & 47.6 & 158 & 1 & 47.60 & 47.6 \\
2 & Diplexer & 0.7 & -0.7 & 50.7 & 0.851 & 158 & 0.32 & 47.9 \\
3 & BPF & 1.0 & -1.0 & 75.1 & 0.794 & 135 & 0.56 & 48.5 \\
4 & 2nd LNA & 1.24 & 17.7 & 95.8 & 58.9 & 107 & 0.89 & 49.4 \\
5 & BPF & 1.0 & -1.0 & 75.1 & 0.794 & 6310 & 0.01 & 49.4 \\
6 & Mixer & 8.0 & -10.0 & 1540 & 0.100 & 5012 & 0.31 & 49.7 \\
7 & SAW & 0.9 & -0.9 & 66.8 & 0.813 & 501 & 0.13 & 49.8 \\
8 & LPF & 1.0 & -1.0 & 75.1 & 0.794 & 407 & 0.18 & 50.0 \\
9 & Rx IC & 3.0 & - & 289 & - & 324 & 0.89 & 50.9 \\
\hline
\end{tabular}

Table 4.3: Noise temperature of L-band receiver excluding antenna feed network evaluated with Friis formula (CubeSat receiver used for link budgets) 


\subsection{Receiver Eb/No Validation}

As stated previously, a receiver's required carrier-to-noise ratio (CNR) establishes the basis for a link budget. For a digital communication link this requirement is set by the bit-error-rate (BER) of a chosen communication protocol which is inversely related to the energy per bit to noise power spectral density $\left(E_{b} / N_{0}\right)$ of a chosen modulation scheme. The best method to validate this relationship is to recreate $B E R$ vs $E_{b} / N_{0}$ curves and compare them to the theoretical curves. In order to recreate these curves a method of measuring $B E R$ for a known $E_{b} / N_{0}$ is required, which requires creating a controlled CNR environment to measure $E_{b} / N_{0}$.

\subsubsection{BERT Method}

A bit-error-rate test (BERT) is an error measurement test which shows the ratio of bit errors received to the total number of bits transmitted, shown in Equation (4.4). In order to complete a BERT, the receiver must have a predetermined knowledge of the correct sequence of bits transmitted. For this L-band link budget validation the sequence used is a pseudorandom number (PN) sequence where the receiver determines the location in the sequence from two previous bytes. For a BER of $10^{-5}$ to be measured a minimum of 100,000 bits must be transmitted.

$$
B E R=\frac{\text { bit errors received }}{\text { total bits transmitted }}=\frac{E}{N}
$$

The ON Semiconductor DVK-2 main board computes BER and includes a display to show the results every 100,000 bits computed for a BER resolution of $10^{-5}$. Figure 4.8 
shows the L-band receiver with DVK-2 main board (green board) and an enlargement of the display showing carrier offset, received carrier power, and computed BER.

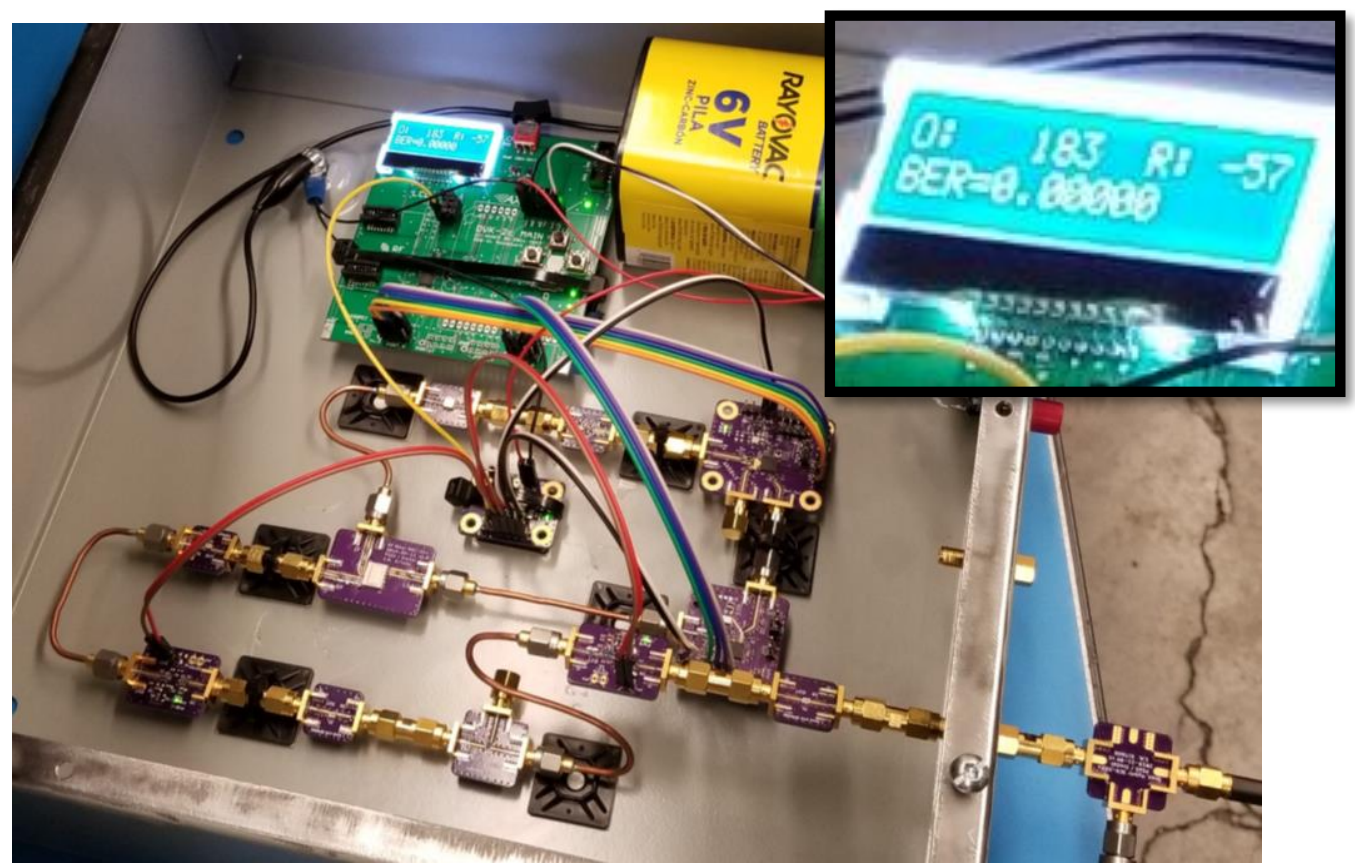

Figure 4.8: L-band receiver showing active DVK-2 main board with BER display

Computed BER is of course not a static value for a given CNR. It fluctuates based on the error rate probability, and therefore a given BER is associated with a confidence level $(\mathrm{CL})$ ratio or percentage where only an infinite number of transmitted bits relates to a $100 \%$ confidence level. For a given number of bits transmitted $(N)$ and the total number of errors received $(E)$ a relationship to confidence level $(C L)$ can be determined with the displayed BER, shown in Equation (4.5) [17].

$$
C L=1-\frac{\sum_{k=0}^{E}\left[\frac{(N \cdot B E R)^{k}}{k !}\right]}{e^{(N \cdot B E R)}}
$$

Table 4.4 below, shows the confidence level for a few displayed BER values on the DVK2 main board for 100,000 bits computed. 


\begin{tabular}{c|c} 
BER Displayed & CL for N = 100k \\
\hline 0.00100 & $47.3 \%$ \\
0.00010 & $41.7 \%$ \\
0.00001 & $26.4 \%$
\end{tabular}

Table 4.4: Confidence level for displayed BER

During BER vs CNR recordings for link budget validation the BER value used was not corrected in an effort to increase confidence level. However, many BER values were recorded and an average taken for every CNR level recorded in an effort to increase confidence level.

\subsubsection{Controlled CNR Environment}

Three methods were used to create controlled CNR levels and record BER. After recording CNR and its associated BER data the feed point to the receiver, pointed out on the following figures, was fed into a spectrum analyzer to measure the corresponding carrier and noise power levels. For power levels close to the spectrum analyzer's noise floor an LNA was used with a Y-factor correction method to accurately resolve power levels. This will be discussed further in the following section.

(1) Closed system bench test with a fixed carrier power and variable noise power, shown below in Figure 4.9. Carrier power was set from the transmitter using attenuation before being fed into a combiner at the receiver with wideband noise generated from a noise generator and adjusted with step attenuation. 


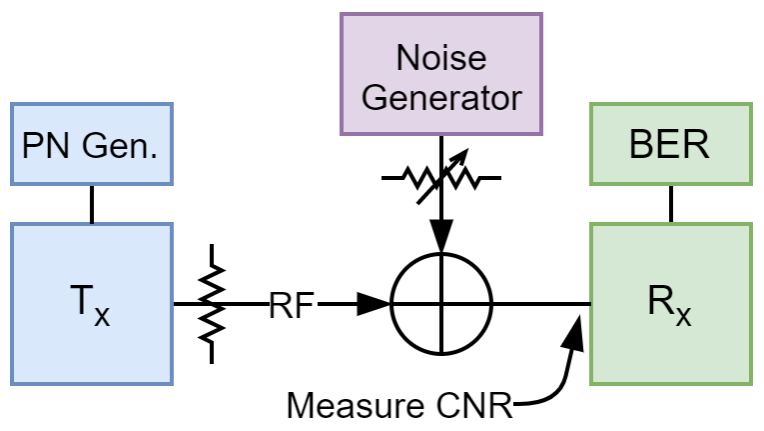

Figure 4.9: Closed system validation test

(2) Antenna system test with ambient noise power and variable carrier power, shown in Figure 4.10. Carrier power was controlled with step attenuation before being fed to an antenna in an anechoic chamber. Antennas were placed about 14 wavelengths away to ensure far-field operation.

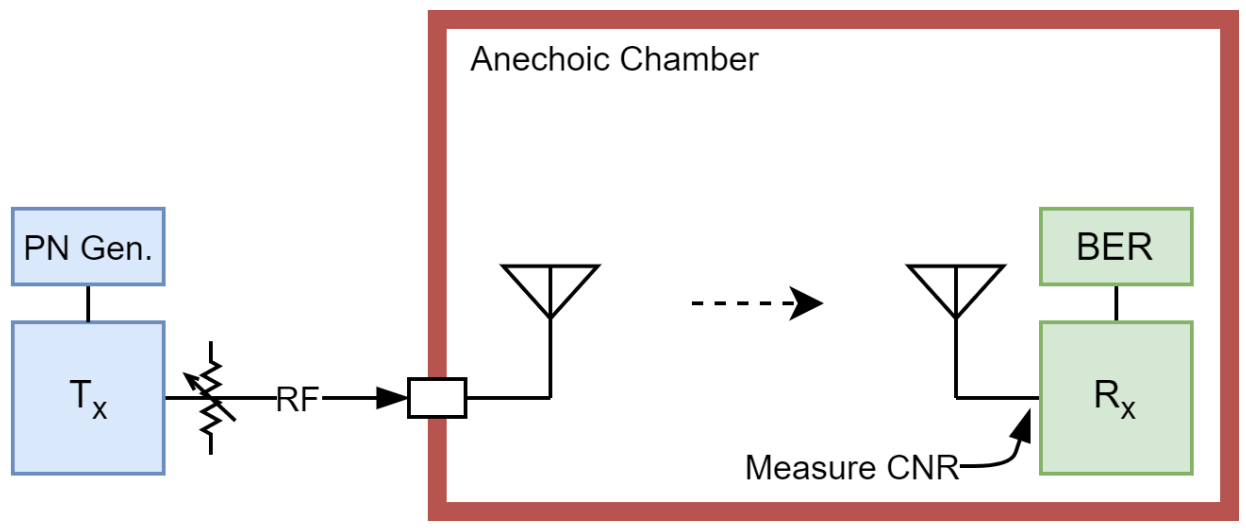

Figure 4.10: Antenna system validation test

(3) Antenna system test with added terrestrial noise power and variable carrier power, shown below in Figure 4.11. Similar to method (2) with the addition of noise power combined into the RF path which mimics wideband terrestrial noise. 


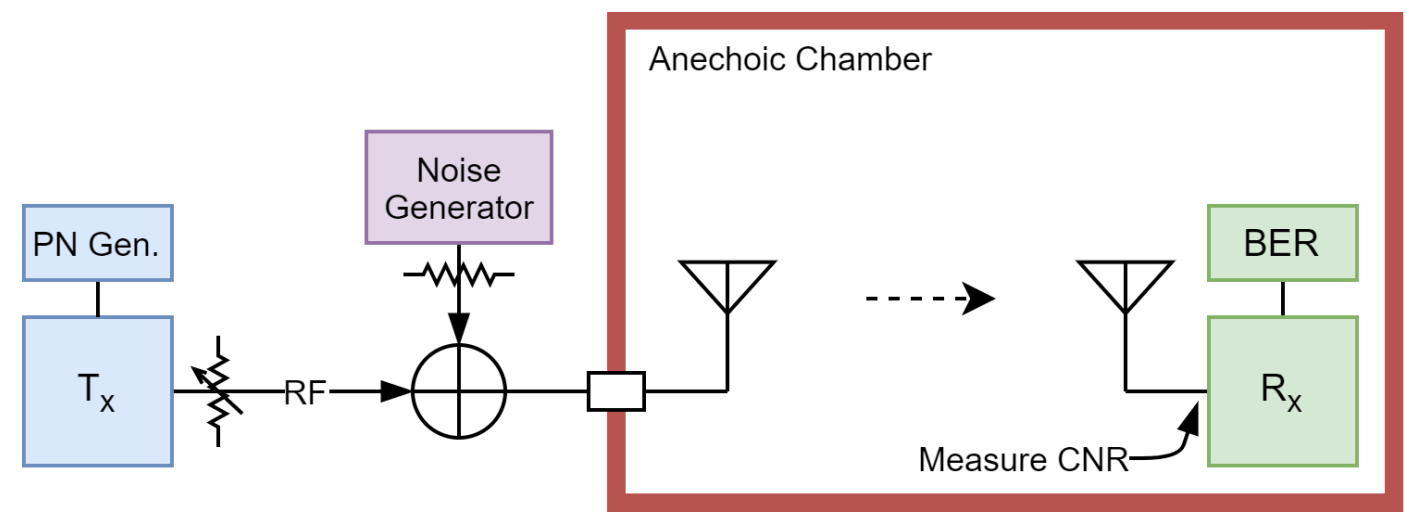

Figure 4.11: Antenna system validation test with terrestrial noise

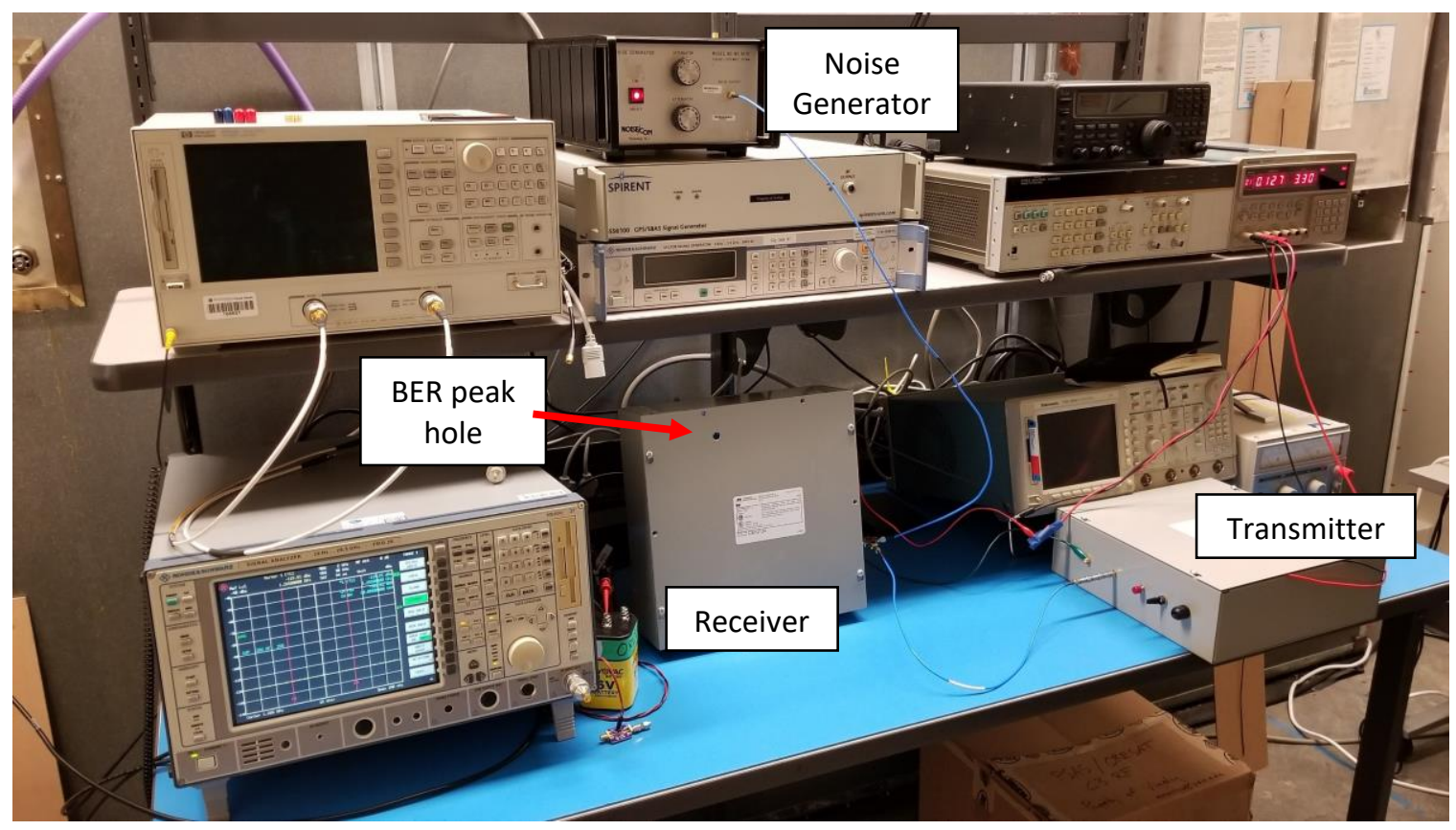

Figure 4.12: Closed system validation test

Figure 4.12 shows a closed system measurement in progress. Figure 4.13 and

Figure 4.14 below show an antenna system measurement in progress where the feed point to the receiver is pointed out and currently connected to a spectrum analyzer to display the received carrier. During CNR measurements, using a spectrum analyzer, an LNA is placed at the receiver CNR measurement point for use with a Y-factor with correction measurement method which is discussed in the following section. 


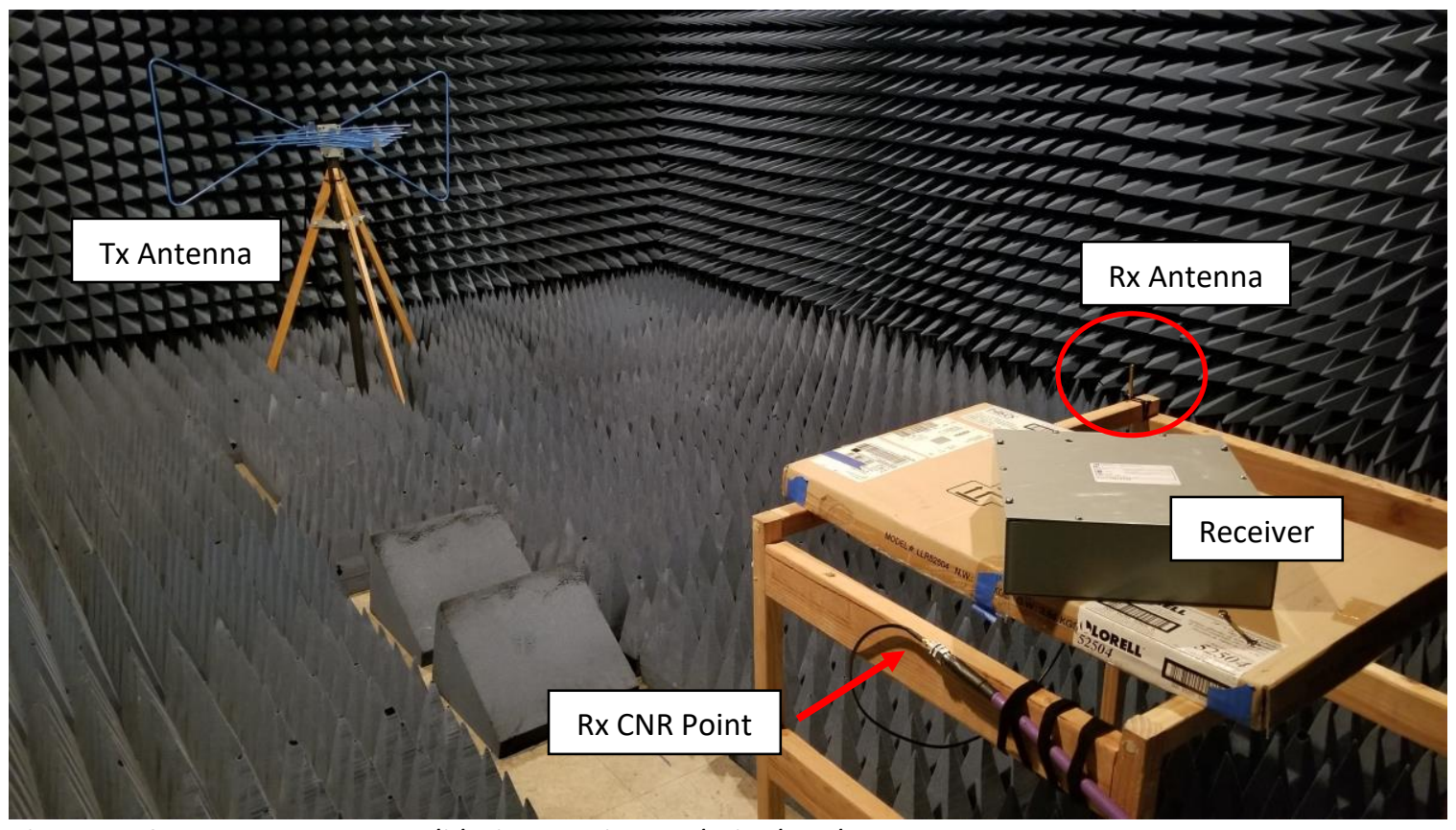

Figure 4.13: Antenna system validation test in anechoic chamber

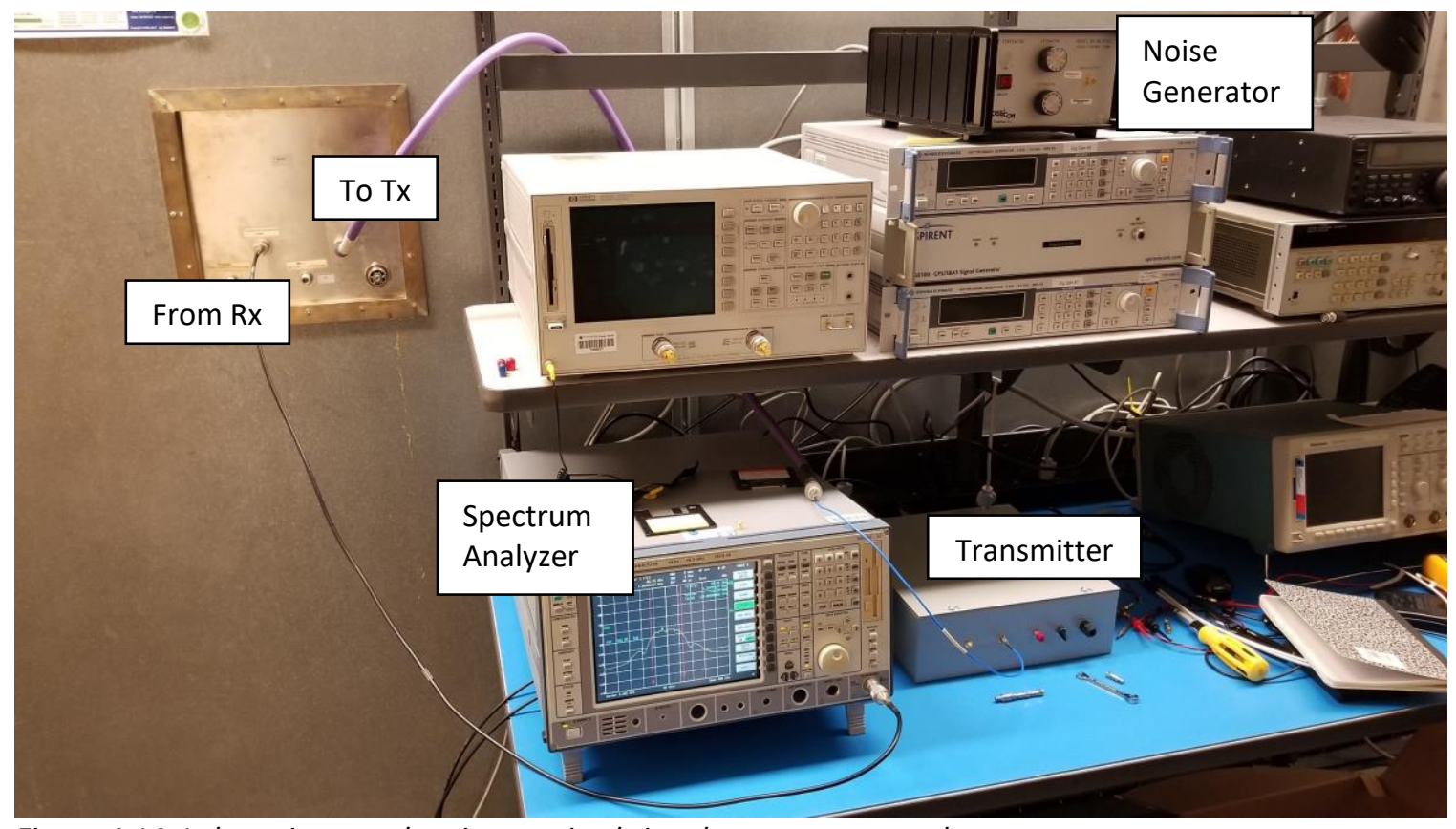

Figure 4.14: Lab equipment showing received signal on spectrum analyzer

\subsubsection{CNR Measurement Method}

To measure the received energy per bit to noise power spectral density $\left(E_{b} / N_{0}\right)$, which directly correlates to the required $\mathrm{E}_{\mathrm{b}} / \mathrm{N}_{0}$ for a link margin $\left(M_{L}\right)$ of zero, a 
controlled carrier-to-noise ratio ( $\mathrm{CNR}$ or $\mathrm{C} / \mathrm{N}$ ) was measured with a spectrum analyzer, and then the dependence of data rate $\left(R_{b}\right)$ and noise channel bandwidth $\left(B_{N}\right)$ were removed. A carrier bandwidth to data rate ratio of $1: 1$ was initially assumed due to using MSK where most of the carrier power is occupied within that bandwidth. This was defined as the channel bandwidth which can also initially be assumed to be the receiver's bandwidth. This relation is shown in Equation (2.31) and reiterated in Equation (4.6) in its logarithmic form.

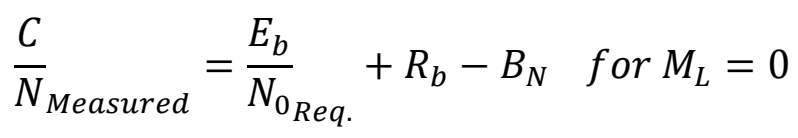

To measure CNR, a Y-factor with correction measurement method was used when either carrier or noise power levels were less than $20 \mathrm{~dB}$ above the spectrum analyzer's noise floor [18]. Equations (4.7) through (4.13) show the formulas involved to use this $\mathrm{Y}$-factor method with a summary of parameters in Table 4.5 below.

$$
\begin{gathered}
P_{C O R}=k+B_{N}+10 \log _{10}\left(T_{C O R}\right) \\
T_{C O R}=\frac{T_{a m b}+T_{L N A}}{10\left({ }^{(C O R / 10}\right)}-T_{L N A} \\
Y_{C O R}=N_{a m b}-P_{\text {meas }}-\delta_{\text {amb }}+\delta_{\text {meas }} \\
\delta_{\text {amb }}=\Delta N_{\text {amb }}-10 \log _{10}\left[10\left({ }^{\Delta N_{\text {amb }} / 10}\right)-1\right] \\
\delta_{\text {meas }}=\Delta \mathrm{P}_{\text {meas }}-10 \log _{10}\left[10\left(\mathrm{P}_{\text {meas } / 10}\right)-1\right] \\
\Delta N_{\text {amb }}=N_{\text {amb }}-N_{\text {floor }} \\
\Delta \mathrm{P}_{\text {meas }}=P_{\text {meas }}-N_{\text {floor }}
\end{gathered}
$$




\begin{tabular}{c|l|l} 
Parameter & \multicolumn{1}{|c}{ Unit } & Description \\
\hline$T_{a m b}$ & $\mathrm{~K}$ & Measured ambient temperature at the antenna or feed point \\
$T_{L N A}$ & $\mathrm{~K}$ & Noise temperature of the LNA \\
$N_{\text {floor }}$ & $\mathrm{dBm}$ & Measured noise floor power of terminated SA input \\
$N_{a m b}$ & $\mathrm{dBm}$ & Measured noise floor power of terminated LNA input \\
$P_{m e a s}$ & $\mathrm{dBm}$ & Measured carrier or noise power connected to the LNA \\
$T_{C O R}$ & $\mathrm{~K}$ & Corrected carrier, noise, or antenna temperature \\
$k$ & $\mathrm{dBm} / \mathrm{HzK}$ & Boltzmann's constant \\
$B_{N}$ & $\mathrm{~dB}-\mathrm{K}$ & Channel or receiver bandwidth \\
$P_{C O R}$ & $\mathrm{dBm}$ & Corrected carrier, noise, or antenna power
\end{tabular}

Table 4.5: Summary of Y-factor with correction method parameters

In addition to the Y-factor correction method, if a measured carrier power level is very weak and close to the input or ambient noise floor, then the input noise power must be removed from the measured carrier value, shown in the RF spectrum example of Figure 4.15 (left). If measuring a very low noise power level where a noise generator is not overwhelming the receiver internally generated noise, then the receiver's noise must be accounted for in the measured CNR, shown in the RF spectrum of Figure 4.15 (middle). Accounting for the receiver's noise transforms the measured noise to the effective system noise power as previously discussed in sections [2.3 Effective System Noise Temperature] and [4.1.4 Noise Figure].

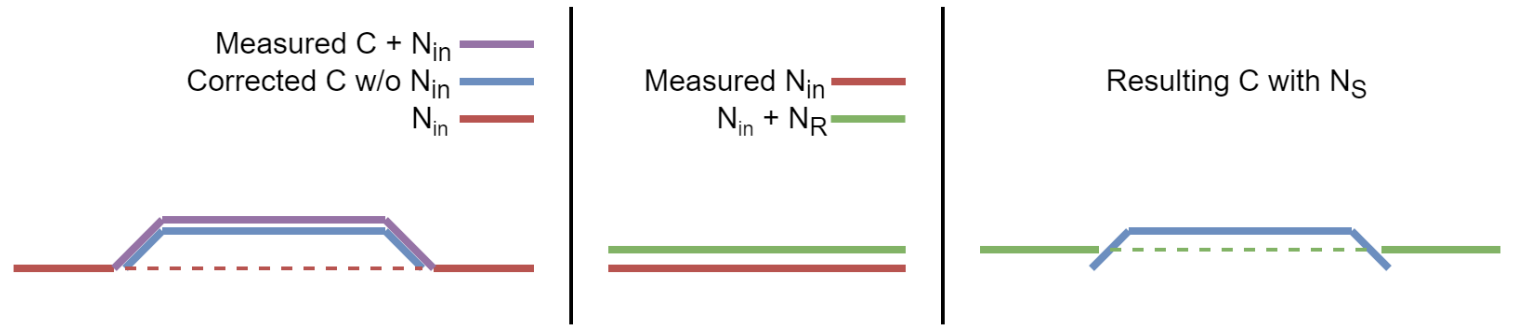

Figure 4.15: RF Spectrum of very weak carrier (left), receiver noise temp. contribution (mid), and resulting corrected carrier power with effective system noise power (right) 
Accounting for these post corrections is accomplished by adding and subtracting the receiver or input/ambient noise temperature from the associated measured noise temperatures. The post corrected temperature measurements are then transformed back to powers. The resulting ratio of the post corrected measured carrier and noise powers is the required carrier to effective system noise power $\left(C / N_{S}\right)$, shown in Equation (4.14). This CNR will be used to evaluate the required $E_{b} / N_{0}$, to be discussed in the following section. The post correction method is summarized in the below equations, (4.15) through (4.18), where $\left(C_{\text {Meas }}^{*}\right)$ and $\left(N_{\text {Meas }}^{*}\right)$ signify the post corrected measured power levels.

$$
\begin{gathered}
\frac{C}{N_{S}}=C_{\text {Meas. }}^{*}-N_{\text {Meas. }}^{*} \\
C_{\text {Meas }}^{*}=k+B_{N}+10 \log _{10}\left[T_{C-\text { meas }}-T_{a m b}\right] \\
N_{\text {Meas }}^{*}=k+B_{N}+10 \log _{10}\left[T_{N-\text { meas }}+T_{R}\right] \\
T_{C-\text { meas }}=10^{\left[\left(C_{\text {Meas }}-k-B_{N}\right) / 10\right]} \\
T_{N-\text { meas }}=10^{\left[\left(N_{\text {Meas }}-k-B_{N}\right) / 10\right]}
\end{gathered}
$$

Since the $\mathrm{Y}$-factor with correction method is being used, the last two equations, (4.17) and (4.18), aren't necessary. The values for $\left(T_{C-\text { meas }}\right)$ and $\left(T_{N-\text { meas }}\right)$ are the respective $\left(T_{C O R}\right)$ from the Y-factor method described above in Equation (4.8) and Table 4.5.

As an example for measuring a very weak carrier, Figure 4.16 below, shows a 60 kbps MSK carrier being measured in a $60 \mathrm{kHz}$ bandwidth using the $\mathrm{Y}$-factor correction method. The channel bandwidth carrier power measures at $-100.3 \mathrm{dBm}$ on the 
spectrum analyzer (SA) (red box) which equates to a corrected channel bandwidth power (after the $\mathrm{Y}$-factor method is applied) of $-120.0 \mathrm{dBm}$. The power spectral density $\left(\mathrm{C}_{0}\right)$ is $-167.8 \mathrm{dBm} / \mathrm{Hz}$ which has an equivalent temperature of 1,208 Kelvin. The added noise power of the environment at an ambient temperature of 294 Kelvin $\left(69.5^{\circ} \mathrm{F}\right)$ has a significant effect on this measurement. The ambient noise power contribution is then removed which gives the true equivalent carrier temperature of 914 Kelvin with a power spectral density of $-169.0 \mathrm{dBm} / \mathrm{Hz}$, and a true channel bandwidth power of $-121.2 \mathrm{dBm}$ in $60 \mathrm{kHz}$. It's important to note here after the ambient power adjustment the carrier power measures $4.93 \mathrm{~dB}$ above the environment noise floor and only $3.14 \mathrm{~dB}$ above the receiver's effective system noise power ( $+150 \mathrm{~K})$ which is used to determine measured CNR and thus $E_{b} / N_{0}$. The corresponding BER for this measurement was 0.18 . Figure 4.17 below, shows the same 60 kbps MSK carrier with an elevated power level which gives an adjusted measured CNR of $16.7 \mathrm{~dB}$ with a corresponding BER of $7.5 \times 10^{-5}$. 


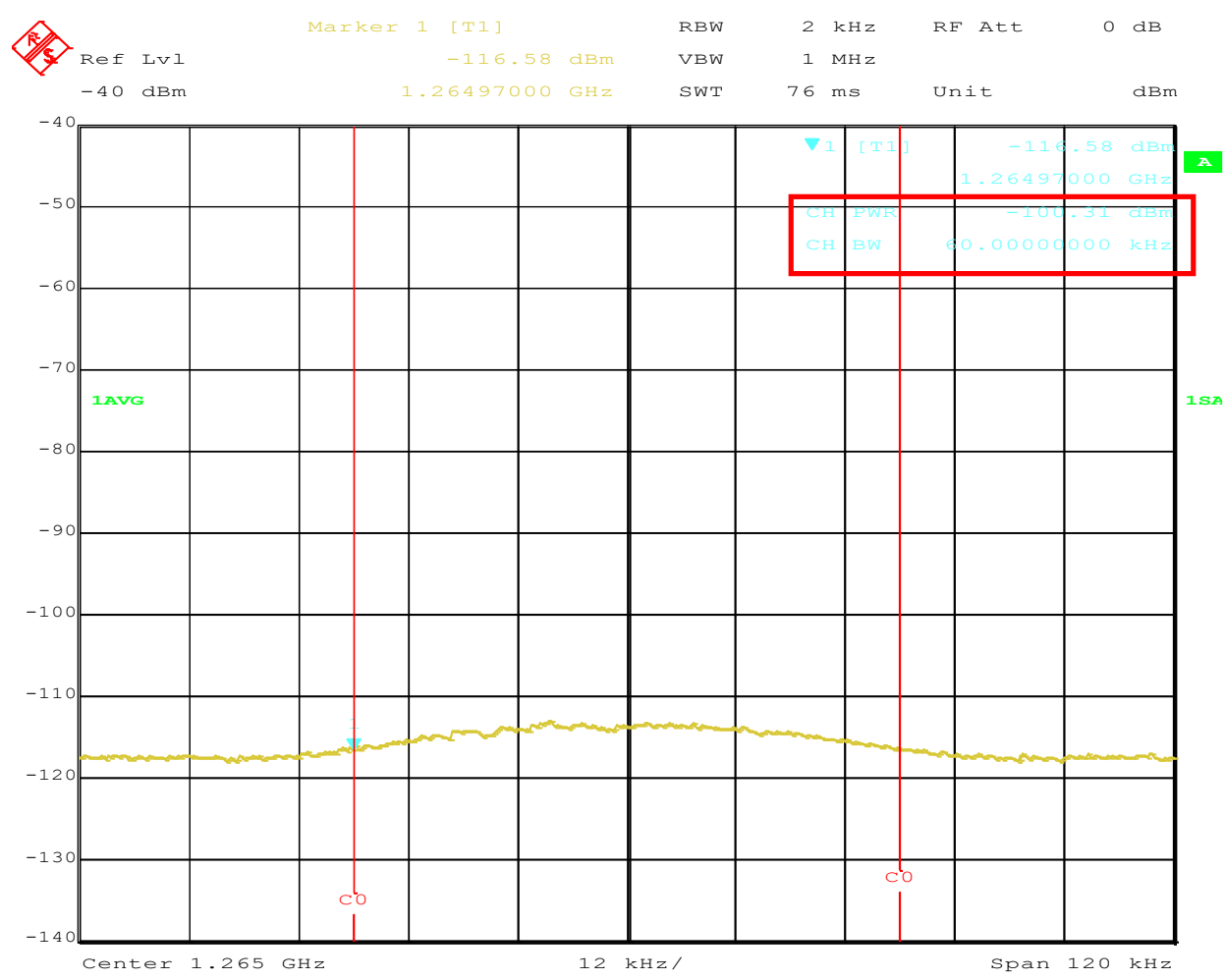

Figure 4.16: Measured very weak 60 kbps MSK with 3.14 dB CNR on SA via Y-factor correction method

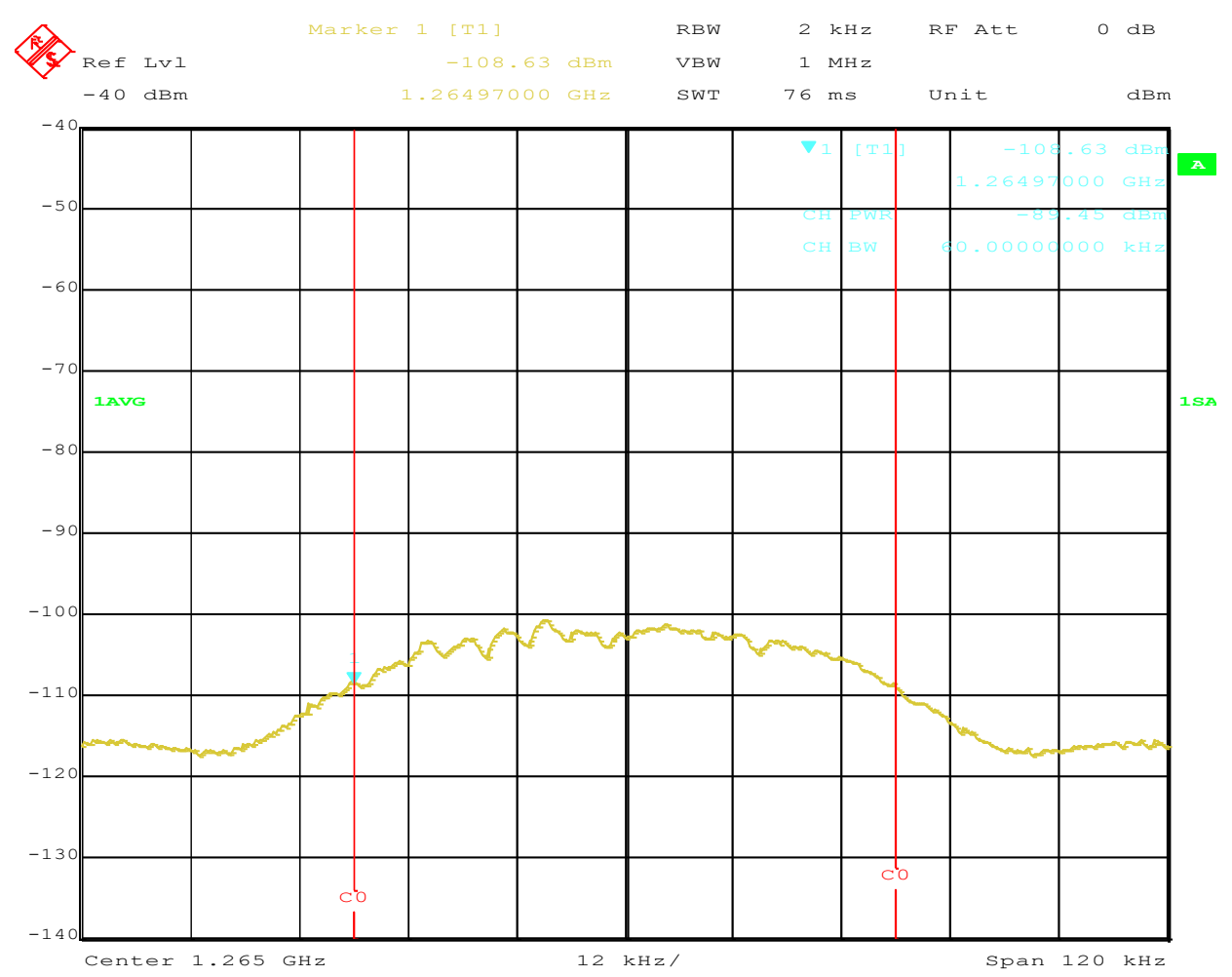

Figure 4.17: Measured 60 kbps MSK with 16.7 dB CNR on SA with Y-factor correction method 


\subsubsection{Receiver Bandwidth Influence}

Throughout the research of this thesis, discrepancies were discovered which raised the possibility of the receiver's bandwidth affecting CNR, and led to question the true relationship of $C N R$ to $E_{b} / N_{0}$ [4] [8]. Does the additional thermal noise contribution from the final bandwidth of the baseband filter affect required CNR? In a modern transceiver IC, the receiver's baseband bandwidth will be set by the PLL's loop-filter and in DSP by with the use of a final FIR filter. If the receiver's bandwidth widens then received carrier power and noise power increase. However, the carrier's channel bandwidth was most likely close to its maximum width, therefore increasing the receiver's bandwidth only increases the received noise power and not the carrier power. Another way of stating this is the noise power increases due to its power spectral density being constant throughout the increasing bandwidth, and the carrier power would not increase due to its power spectral density decreasing throughout the increasing bandwidth.

According to the AX5043 transceiver IC datasheet, the receiver's PLL loop-filter is $500 \mathrm{kHz}$. Since this filter is much wider than the data rate bandwidth an additional final baseband filter is implemented. The default value for this filter was set to $1.5 x$ the data rate. Figure 4.18 below, shows the final baseband receiver filter relative to the data rate in the screenshot from ON Semiconductor's AX-RadioLAB software application [15]. 


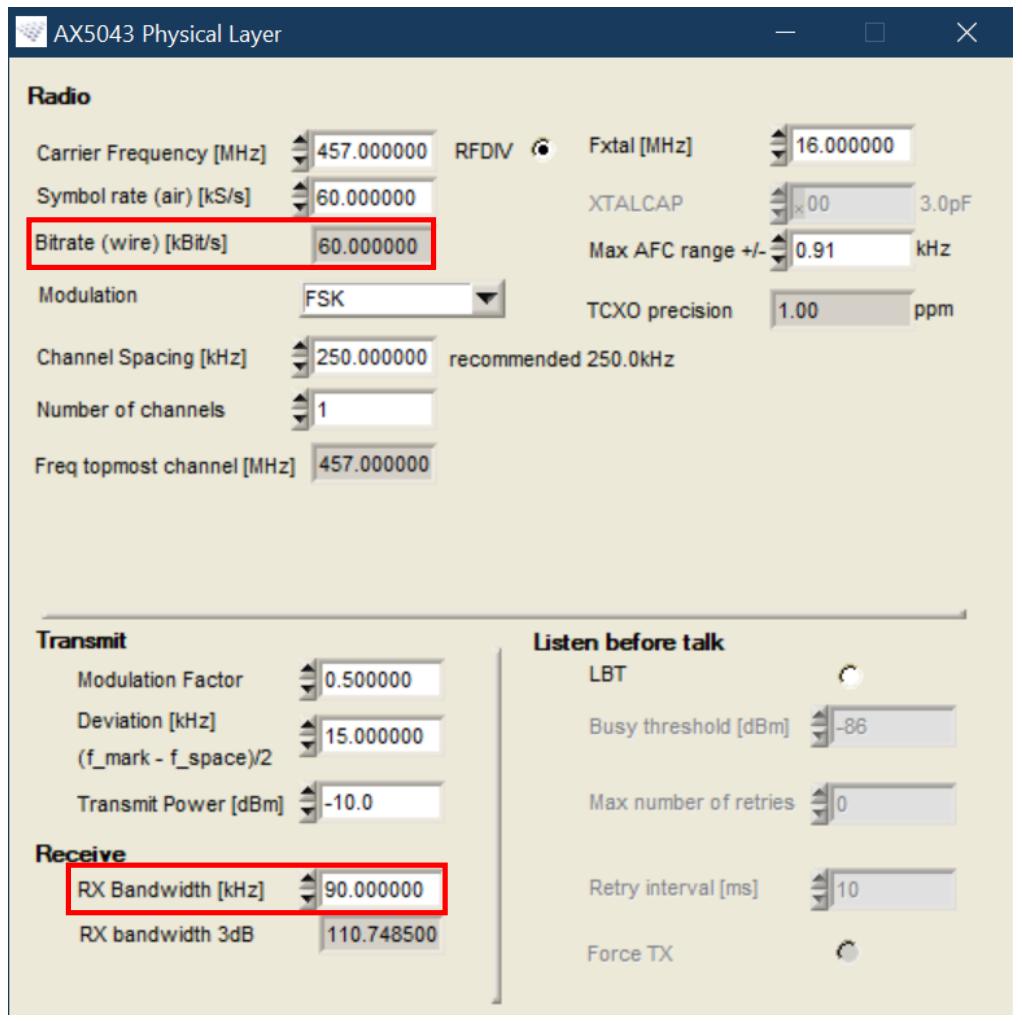

Figure 4.18: AX5043 transceiver IC radio parameters shown in AX-RadioLAB software application

The two known relationships for carrier-to-noise ratio $\left(C /{ }_{N}\right)$ to energy per bit to noise power spectral density $\left(E_{b} / N_{0}\right)$ have been with a dependency of data rate $\left(R_{b}\right)$ or the receiver's bandwidth $\left(B_{R}\right)$, Equations (4.19) and (4.20) respectively show these relationships in logarithmic form.

$$
\begin{aligned}
& \frac{C}{N}=\frac{E_{b}}{N_{0}}+R_{b}-B_{N} \\
& \frac{C}{N}=\frac{E_{b}}{N_{0}}+B_{R}-B_{N}
\end{aligned}
$$

The channel bandwidth in these equations is actually the receiver bandwidth, however, it is written as the former to show distinction that it applies to the noise 
bandwidth and they cancel on both sides of the equations. This reveals the carrier to noise power spectral density ratio $\left(C / N_{0}\right)$, shown below in Equations (4.21) and (4.22).

$$
\begin{gathered}
\frac{C}{N_{0}}=\frac{E_{b}}{N_{0}}+R_{b} \\
\frac{C}{N_{0}}=\frac{E_{b}}{N_{0}}+B_{R}
\end{gathered}
$$

An initial experiment is discussed in this section which utilizes two different receiver bandwidths to verify the best relationship. The data rate method, Equation (4.21), was used first to compute $\mathrm{E}_{\mathrm{b}} / \mathrm{N}_{0}$ in a closed system test for 60 kbps MSK with a constant carrier power set to $-90 \mathrm{dBm}$. Figure 4.19 shows the data rate method results for a 60 and $120 \mathrm{kHz}$ receiver bandwidths. The receiver bandwidth method, Equation (4.22), was used second to compute $\mathrm{E}_{\mathrm{b}} / \mathrm{N}_{0}$ from the data previously collected, shown in Figure 4.20.

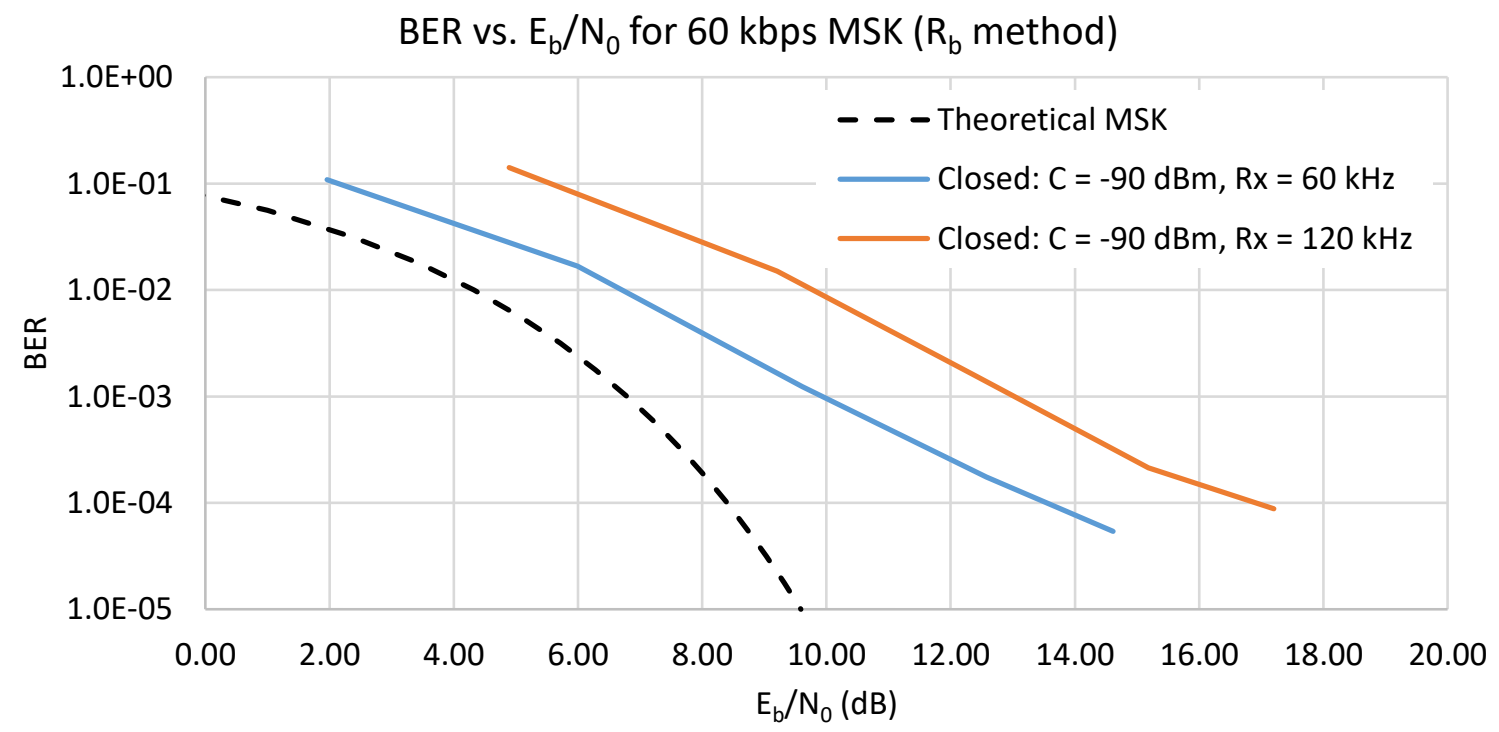

Figure 4.19: BER vs. $E_{b} / N_{0}$ plot for 60 kbps MSK using data rate method 


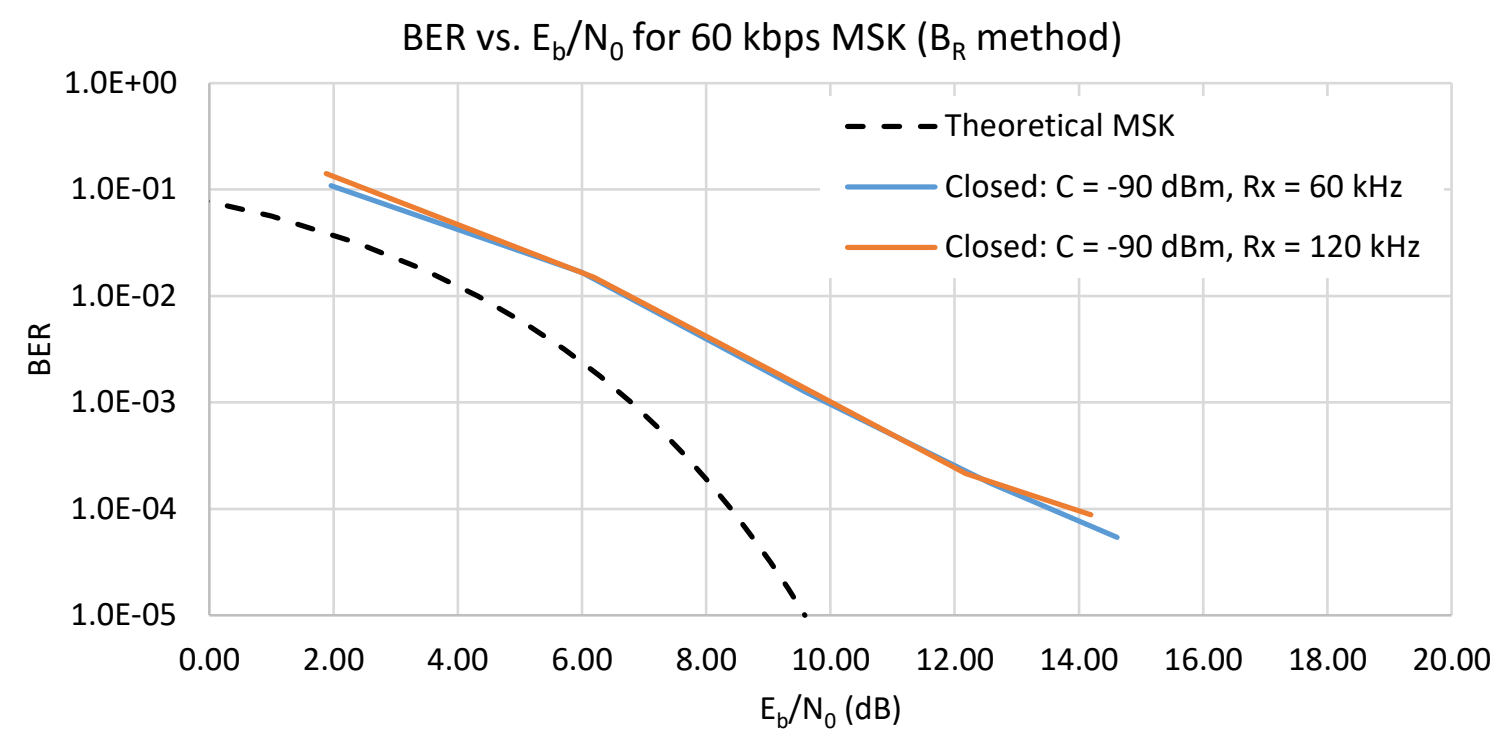

Figure 4.20: BER vs. $E_{b} / N_{0}$ plot for 60 kbps MSK using receiver bandwidth method

The $E_{b} / N_{0}$ curves mostly overlap in the receiver bandwidth method which prove to be the best relationship for this configuration, however, a large modulation implementation loss is noticed. Modulation implementation loss $\left(L_{M o d}\right)$ is defined as the difference of measured $E_{b} / N_{0}$ to theoretical $E_{b} / N_{0}$ for a given $B E R$, shown below in Equation (4.23). Figure 4.21, below, shows the evaluated implementation loss from the receiver bandwidth method.

$$
L_{\text {Mod }}={\frac{E_{b}}{N_{0_{\text {Meas }}}}}-{\frac{E_{b}}{N_{0}}}_{\text {Theory }}
$$




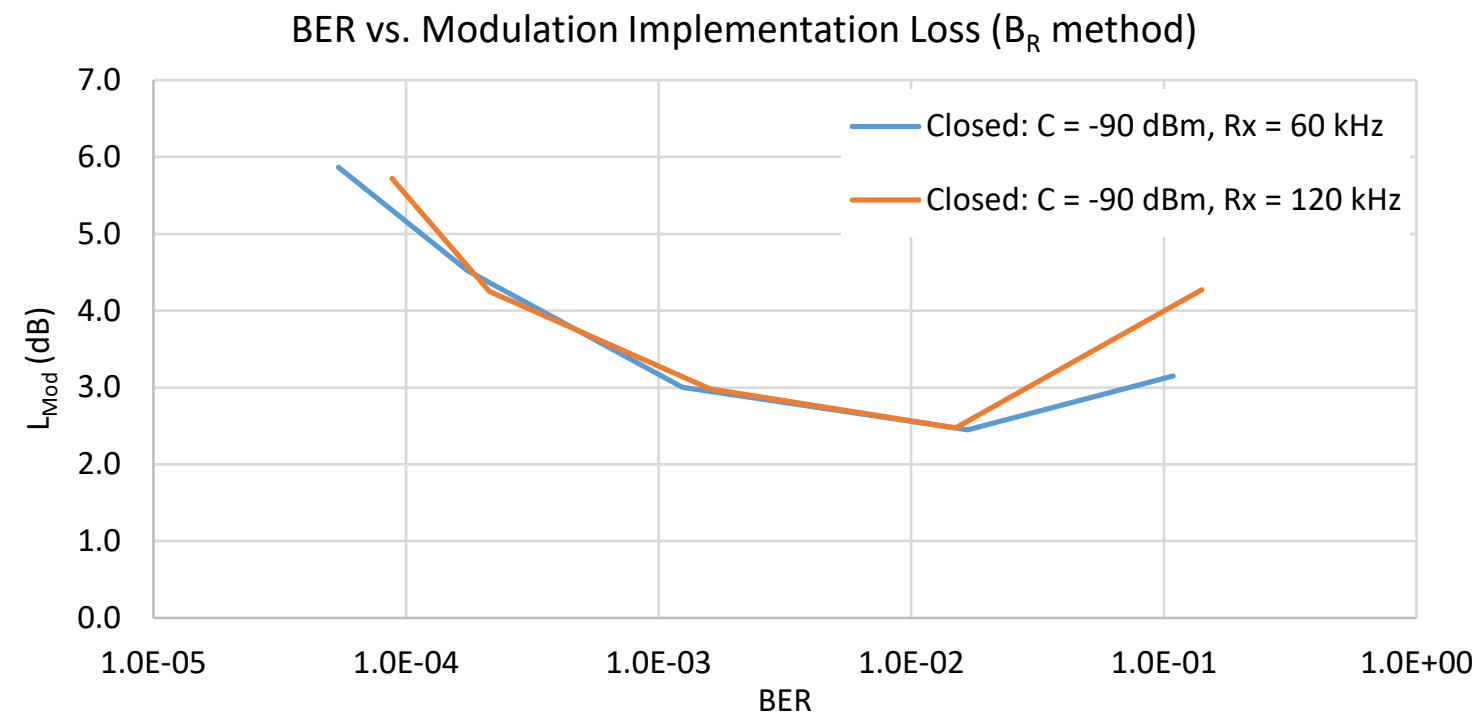

Figure 4.21: Modulation implementation loss for receiver bandwidth method

A revised $E_{b} / N_{0}$ data rate method relationship with additional dependences to the receiver's bandwidth to data rate ratio and modulation index $\left(h_{m}\right)$ was discovered and proved worthy throughout the remaining data analyzed, shown in Equation (4.24) where terms in brackets are non-logarithmic, and with the ratio of receiver bandwidth to data rate shown in Equation (4.25). This relationship worked well for all binary frequency-shift keying (BFSK) modulation schemes where the modulation index was less than or equal to 0.75 and greater than or equal to 0.5 . It is possible the revised method works with an index greater than 0.75 but less than 1.0 as well, however, this was not tested.

$$
\begin{gathered}
\frac{C}{N_{0}}=\frac{E_{b}}{N_{0}}+R_{b}+10 \log _{10}\left[\Delta B_{R}\left(2-h_{m}\right)\right] \quad \text { for } 0.5 \leq h_{m} \leq 0.75 \\
\Delta B_{R}=\frac{B_{R}}{R_{b}}
\end{gathered}
$$


This revised method was computed with data previously collected for the original methods and shown below in Figure 4.22. The $E_{b} / N_{0}$ curves overlap better and is shifted closer to the theoretical curve with about $1.5 \mathrm{~dB}$ implementation loss for BER values greater than $10^{-3}$, shown below in Figure 4.23 .

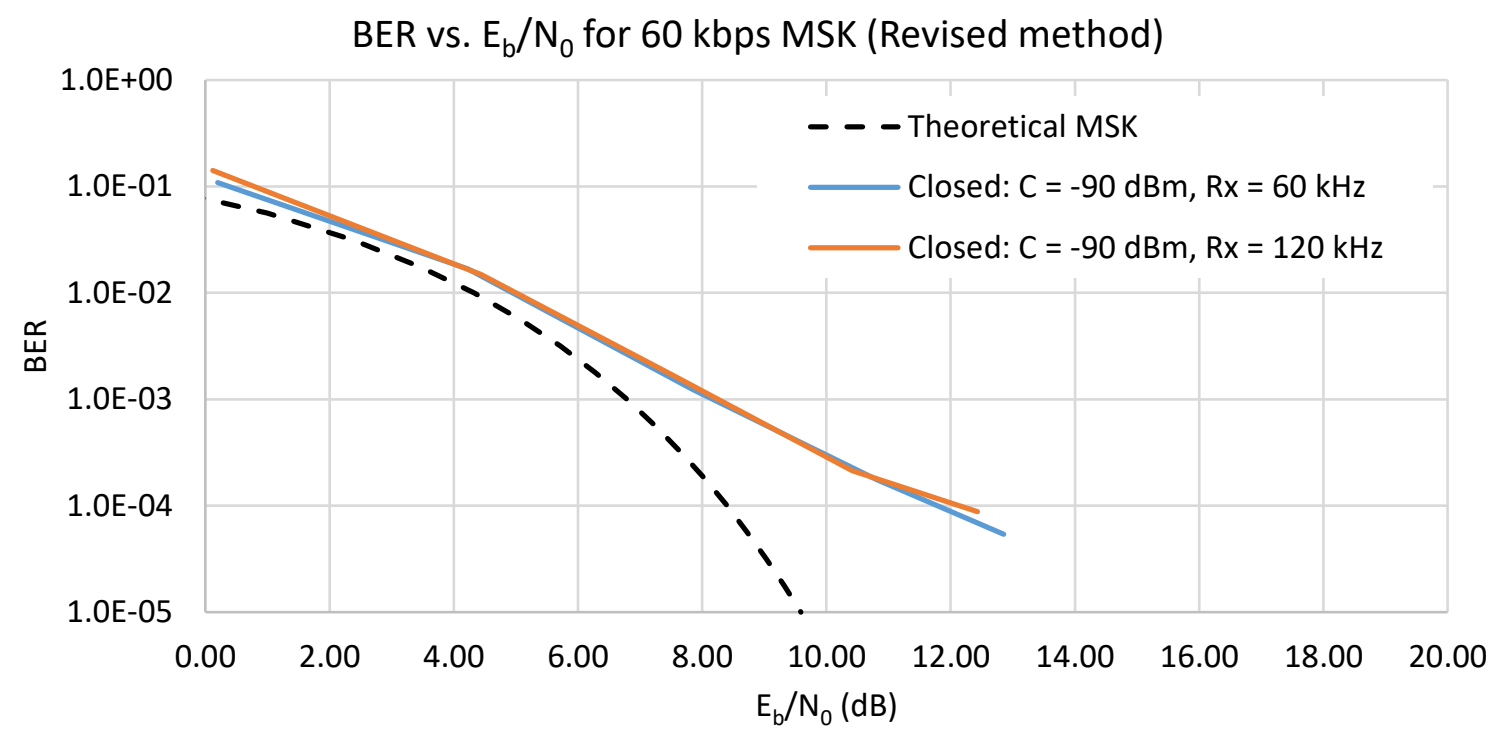

Figure 4.22: BER vs. $E_{b} / N_{0}$ plot for 60 kbps MSK using revised $C N R$ to $E_{b} / N_{0}$ relationship

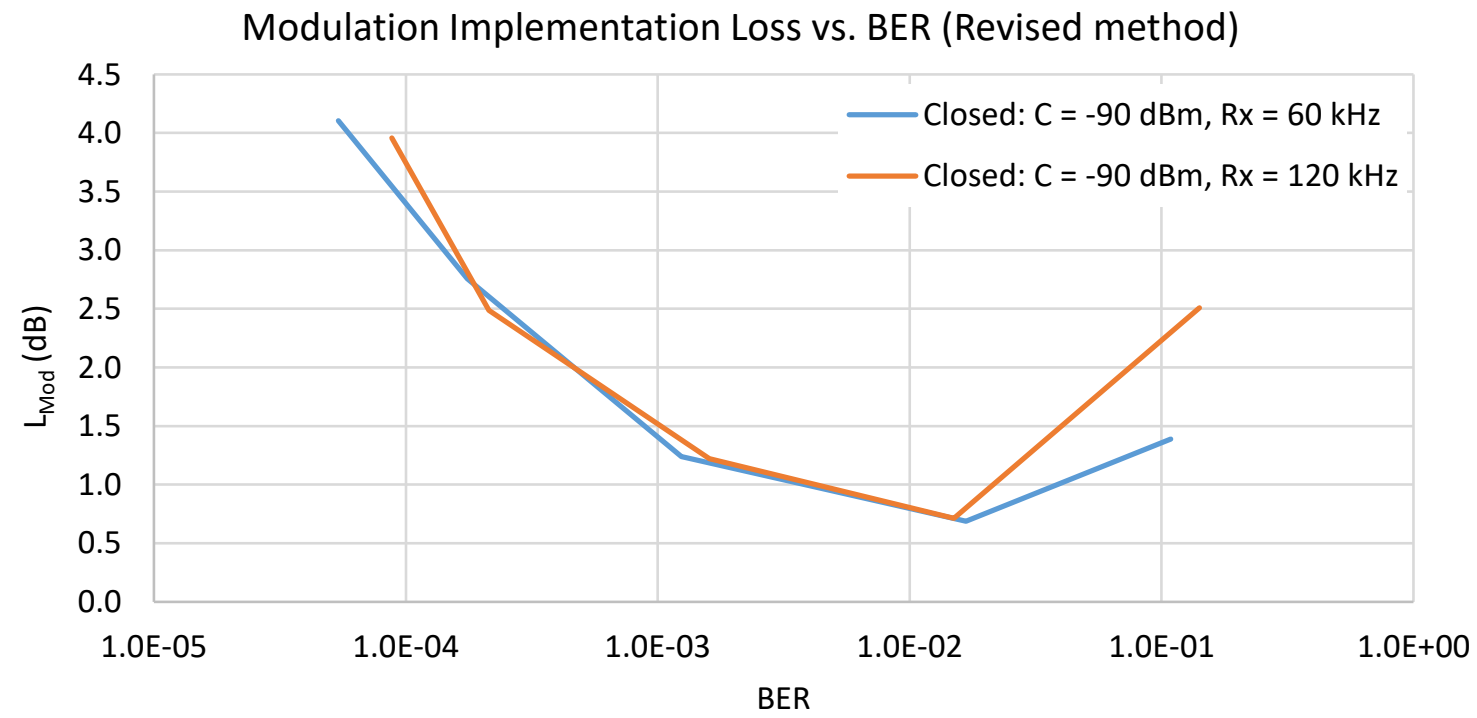

Figure 4.23: Modulation implementation loss for revised method 
In addition, the $B E R$ to $E_{b} / N_{0}$ relationship, initially introduced in [2.4 Digital Modulation], for modulation indices between 0.5 and 0.75 proved to match the relationship with an index of 0.5 (MSK). This formula was first shown in Equation (2.32) and is shown again for convenience in Equation (4.26) below.

$$
B E R=Q\left(\sqrt{\frac{2 E_{b}}{N_{0}}}\right) \quad \text { for FSK with } 0.5 \leq h_{m} \leq 0.75
$$

\subsubsection{Final Validation Formulas}

A communication system link budget validation comes down to validating the digital demodulation of the receiver. The process of relating the receiver's measured carrier power to effective system noise power. For link budget analysis this happens at the antenna's terminals; however, for accuracy in the lab this CNR was determined at the front of the receiver where feed line is already deducted. Equations (4.27) through (4.30) show the final relationships used to validate the prototype CubeSat L-band communication system, shown in logarithmic form except for the terms in brackets. The additional factor discovered for the $\mathrm{E}_{\mathrm{b}} / \mathrm{N}_{\mathrm{o}}$ formula will be referred to as the receiver's efficiency factor $\left(F_{R}\right)$.

$$
\begin{gathered}
\frac{C}{N_{S}}=C_{\text {Meas }}^{*}-N_{0-\text { Meas }}^{*}-B_{N}=\frac{E_{b}}{N_{0}}+R_{b}+F_{R}-B_{N} \\
C_{\text {Meas }}^{*}=k+B_{R}+10 \log _{10}\left[T_{C-\text { meas }}-T_{\text {amb }}\right] \\
N_{0-\text { Meas }}^{*}=k+10 \log _{10}\left[T_{N-\text { meas }}+T_{R}\right] \\
F_{R}=10 \log _{10}\left[\Delta B_{R}\left(2-h_{m}\right)\right]
\end{gathered}
$$


As stated previously, the channel bandwidth in Equation (4.27) is actually the receiver bandwidth, however, it's written as the former to show distinction that it applies to the noise bandwidth and they cancel on both sides of the equation. During CNR measurements a channel bandwidth was chosen to measure thermal noise power and then transformed to noise spectral density for ease during calculations. In addition, the receiver bandwidth was always used for carrier power measurements. Equations (4.31) through (4.34) were used to compute $E_{b} / N_{0}$ where all parameters are in their logarithmic form except for those in brackets, and $\left(T_{C-\text { meas }}\right)$ and $\left(T_{N-\text { meas }}\right)$ are the respective $\left(T_{C O R}\right)$ from the Y-factor method.

$$
\begin{gathered}
\frac{E_{b}}{N_{0}}=C_{\text {Meas }}^{*}-N_{0-\text { Meas }}^{*}-R_{b}-F_{R} \\
C_{\text {Meas }}^{*}=k+B_{R}+10 \log _{10}\left[T_{C-\text { meas }}-T_{a m b}\right] \\
N_{0-\text { Meas }}^{*}=k+10 \log _{10}\left[T_{N-\text { meas }}+T_{R}\right] \\
F_{R}=10 \log _{10}\left[\Delta B_{R}\left(2-h_{m}\right)\right]
\end{gathered}
$$

\subsubsection{Validation Results}

The prototype CubeSat L-band communication system was tested exhaustively. As discussed in [4.2.2 Controlled CNR Environment] all three methods for system validation were used. In addition, a couple 'no conversion' (NC) configurations were measured with the closed system method. The NC configuration does not include any front-end components to the transceiver ICs in an effort to identify effects of frequency conversion on bit-error-rate (BER). 
The system was first configured for use with a low data rate to achieve a baseline of performance. A 9,600 bps data rate was chosen for use with BFSK and a modulation index of 0.625 . This modulation scheme resembles G3RUH, created by James Miller, and is commonly used in the Amateur radio community including for packet radio beacons [19]. The main difference to G3RUH is the occupied bandwidth of this implementation is wider. Figure 4.24 shows the results of this configuration in various test methods.

BER vs. $E_{b} / N_{0}$ for $9.6 \mathrm{kbps} B F S K, h=0.625, B_{R}=14.4 \mathrm{kHz}$

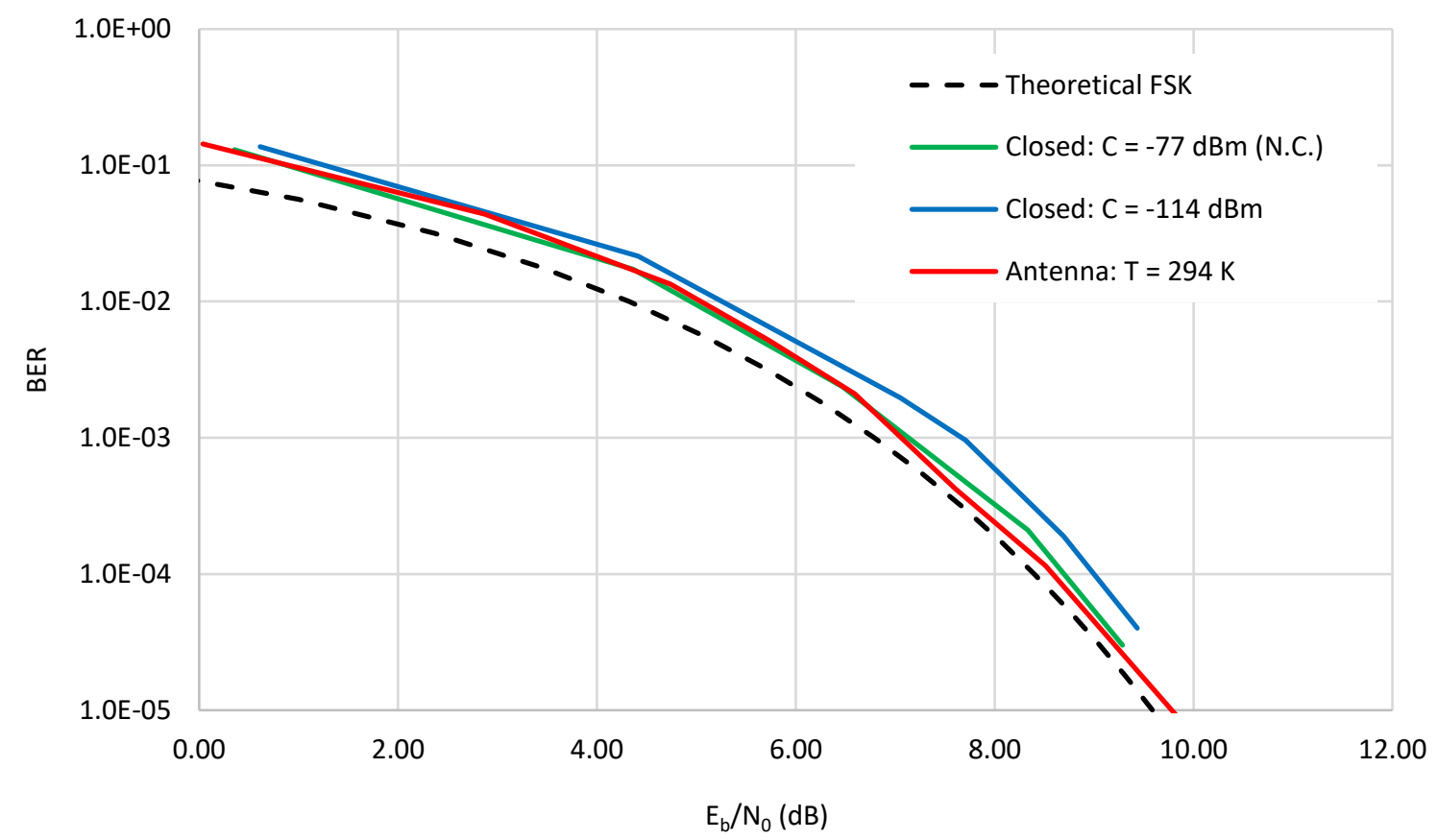

Figure 4.24: $B E R$ vs. $E_{b} / N_{0}$ plot for $9.6 \mathrm{kbps} B F S K, h=0.625$ for various test methods

The system was next configured to match the median data rate of the AX5043 transceiver IC. A 60 kbps MSK (index of 0.5) configuration was chosen with a few varieties of receiver bandwidths, shown below in Figure 4.25. In the plot, many of the curves stop following theoretical values between a BER of $10^{-2}$ and $10^{-3}$ with the no 
conversion (NC) measurement following the longest. The deviation is most likely due to an increase in data rate or a decrease in modulation index caused by internal performance limitations of the AX5043 transceiver IC.

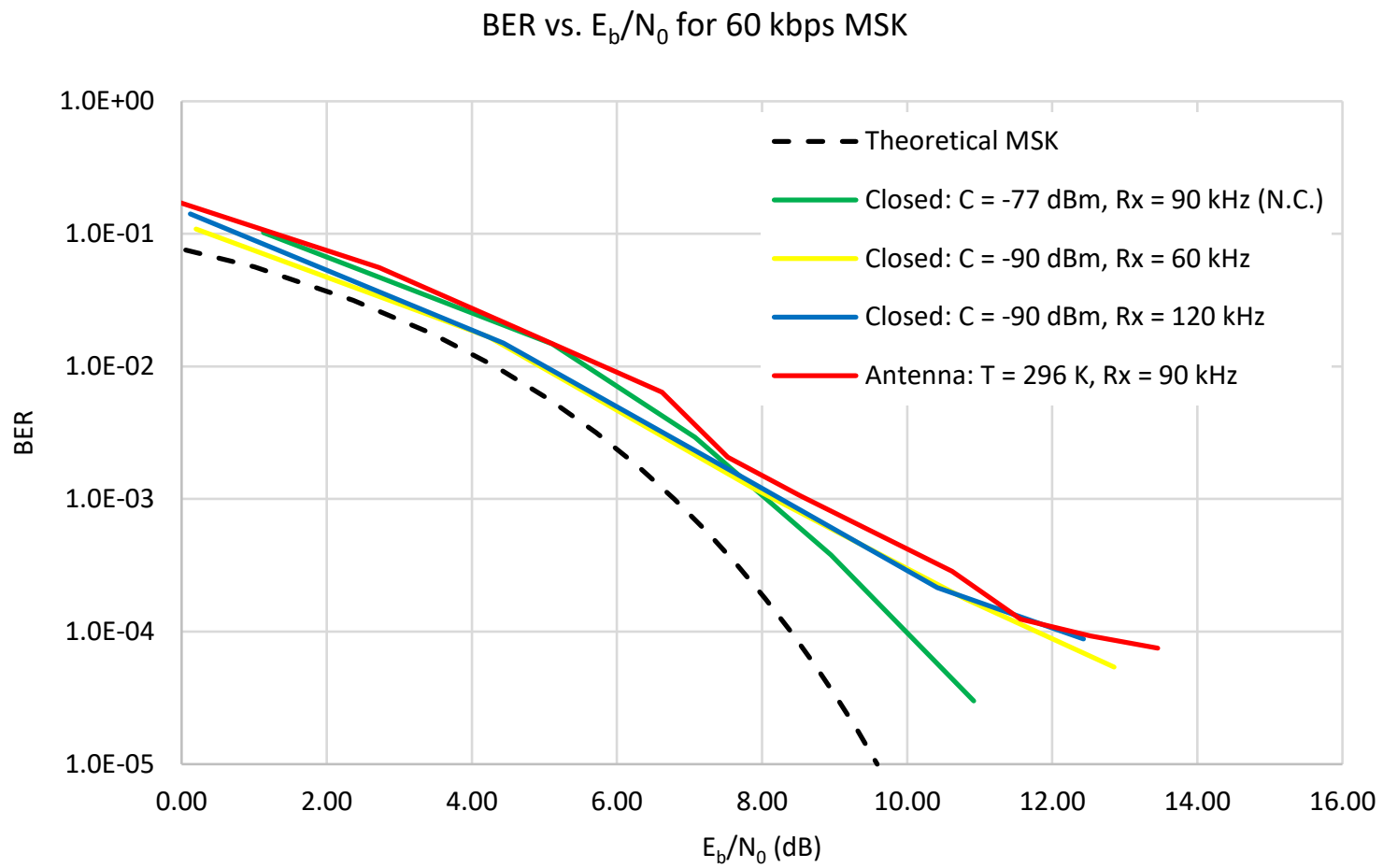

Figure 4.25: BER vs. Eb/No plot for 60 kbps MSK for various test methods

The system was next configured with double the data rate and the same modulation scheme of MSK. This was the only configuration used for antenna system test with 'terrestrial noise' injected into the anechoic chamber along with the carrier, shown below in Figure 4.26. The noise power levels correlated to the two above ambient temperatures were measured at the end of the antenna feed as discussed in [4.2.2 Controlled CNR Environment]. This means the antenna's noise temperature was slightly higher than the measured values due to the feed loss. As seen prior for $60 \mathrm{kbps}$ 
MSK, the $120 \mathrm{kbps}$ MSK has even more degraded performance with the curves starting to deviate at a BER of $10^{-2}$.

BER vs. $E_{b} / N_{0}$ for $120 \mathrm{kbps} M S K, B_{R}=180 \mathrm{kHz}$

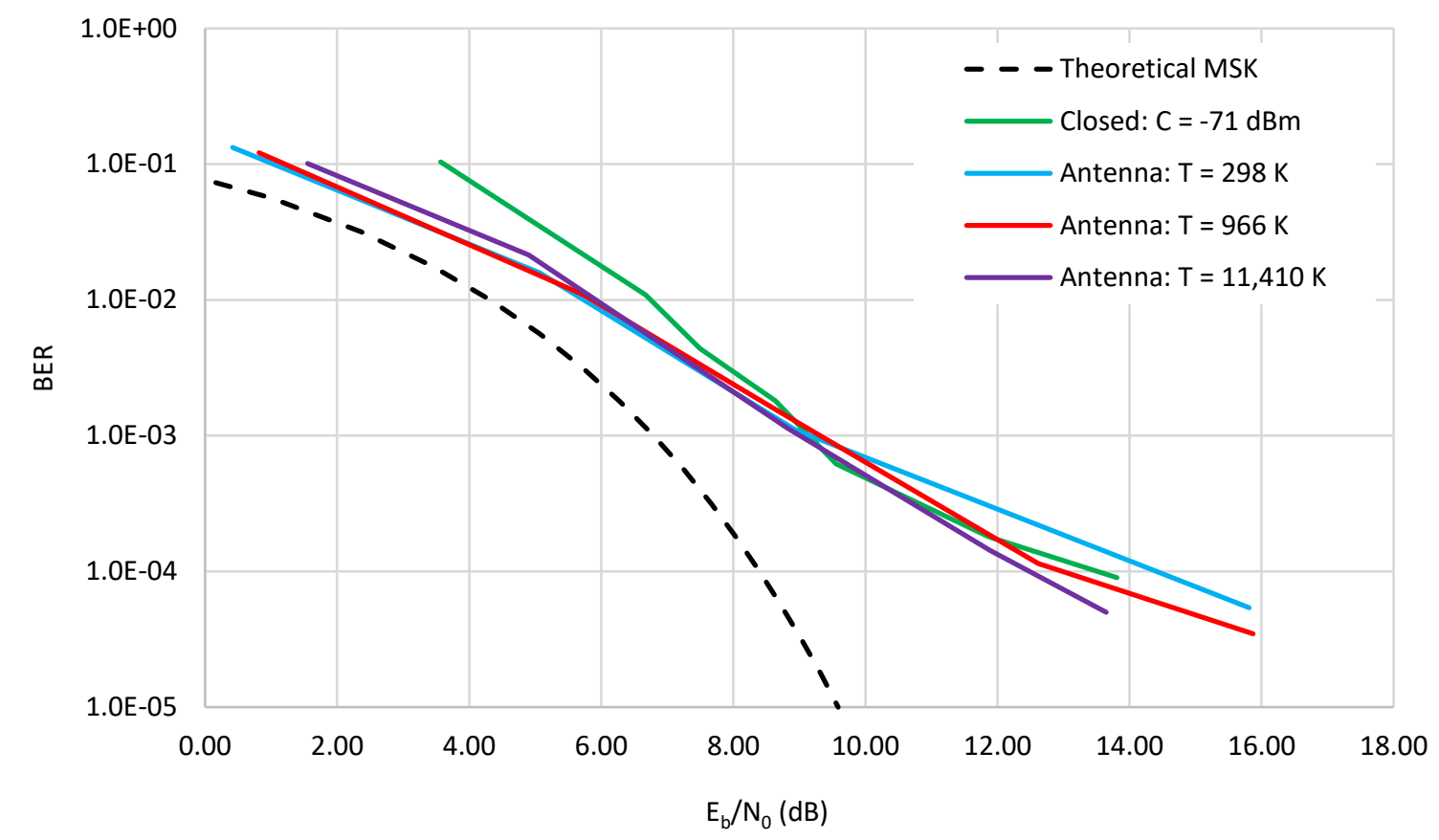

Figure 4.26: BER vs. Eb/No plot for 120 kbps MSK for various test methods

In Figure 4.26 it can also be observed that the closed system measurement (green curve) does not fit well with the others above a BER of $10^{-3}$. This was due to the carrier power level not being low enough relative to the ambient noise floor for a specific data rate and injecting too much noise into the system. This effect was discovered from trial and error and after the discovery a relative carrier power of no more than $40 \mathrm{~dB}$ above the ambient noise floor was chosen for a given data rate for closed system tests.

The system was next configured with a $60 \mathrm{kbps}$ BFSK scheme with an index of 0.75 to further investigate the revised $C N R$ to $E_{b} / N_{0}$ relationship, shown in Figure 4.27. 
The $75 \mathrm{kHz}$ receiver bandwidth curve does not match the revised formula well and does not overlap the $150 \mathrm{kHz}$ variation. The revised $\mathrm{E}_{\mathrm{b}} / \mathrm{N}_{0}$ formula should provide a constant $E_{b} / N_{0}$ with varying receiver bandwidth. After a little investigation it was noticed the receiver bandwidth to frequency deviation was the lowest for any of the other modulation schemes chosen, shown in Table 4.6. Where the total frequency deviation is a function of data rate and modulation index, shown below in Equation (4.35).

BER vs. $E_{b} / N_{0}$ for 60 kbps BFSK, $h_{m}=0.75$

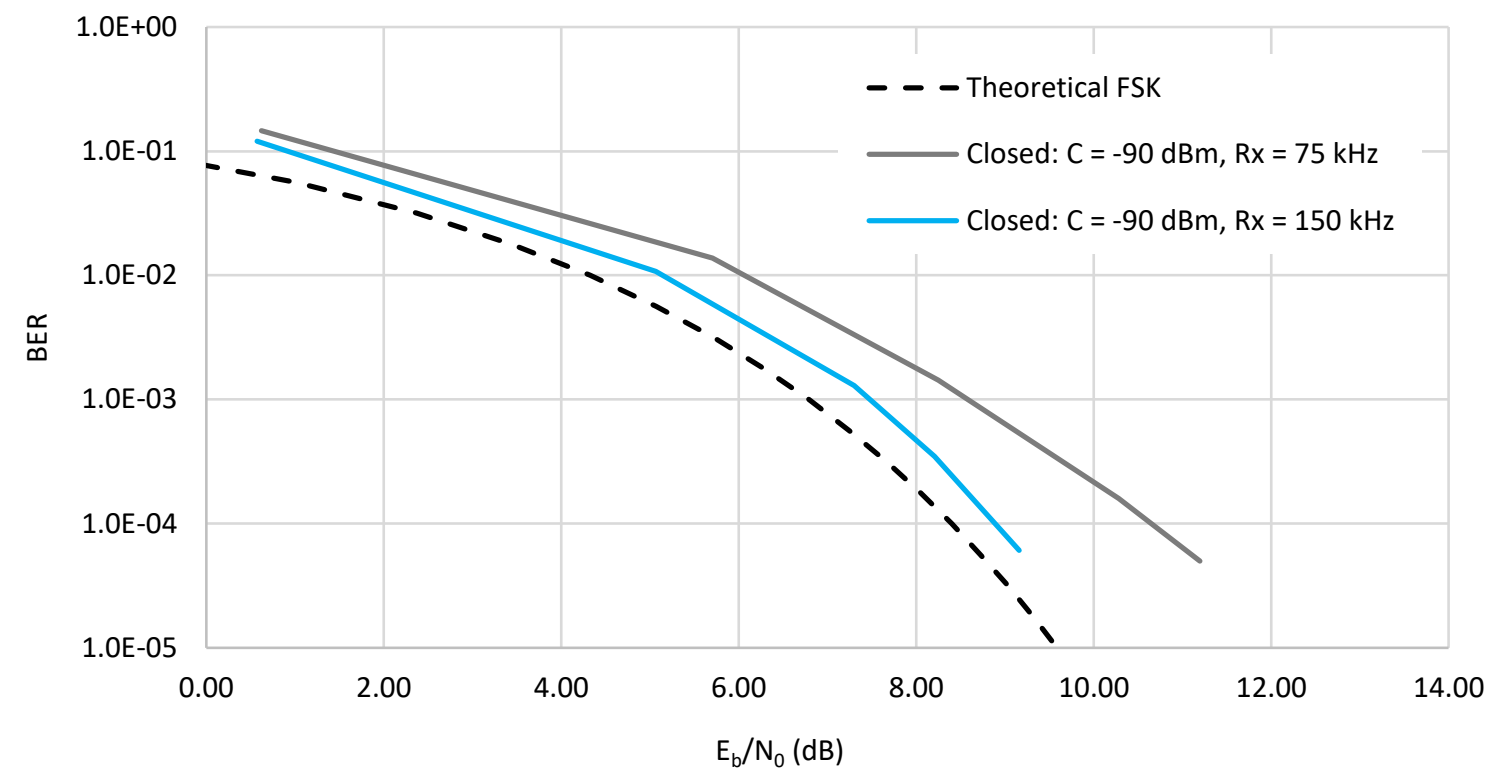

Figure 4.27: BER vs. $E_{b} / N_{0}$ plot for 60 kbps $B F S K, h=0.75$ for various test methods

\begin{tabular}{c|c|c|c|c} 
Index $\left(\mathrm{h}_{\mathrm{m}}\right)$ & $\mathrm{Rb}_{\mathrm{b}}(\mathrm{kbps})$ & $\mathrm{B}_{\mathrm{R}}(\mathrm{kHz})$ & Deviation $(\mathrm{kHz})$ & $\mathrm{B}_{\mathrm{R}} / \mathrm{Dev}$ \\
\hline 0.5 & 60 & 60 & 30 & 2 \\
0.5 & 60 & 90 & 30 & 3 \\
0.5 & 60 & 120 & 30 & 4 \\
0.5 & 120 & 180 & 60 & 3 \\
0.625 & 9.6 & 14.4 & 6 & 2.4 \\
\hline 0.75 & 60 & 75 & 45 & 1.67 \\
\hline 0.75 & 60 & 150 & 45 & 3.33
\end{tabular}

Table 4.6: Summary of receiver bandwidth to deviation for all modulation schemes 


$$
\text { Total Deviation }=R_{b} h_{m}
$$

As noticed above in Table 4.6, the $75 \mathrm{kHz}$ receiver bandwidth for this configuration may be too narrow for the minimum receiver bandwidth, where effectively not enough of the energy-per-bit is being captured. It appears a $B_{R} /$ Dev ratio of greater than two performs best for the AX5043 in BFSK modes.

The influence of the modulation index on $E_{b} / N_{0}$ curves is also observed in the above four figures. It appears the curves with an index greater than 0.6 follow the theoretical $E_{b} / N_{0}$ curves the best. This is most noticeable between the $60 \mathrm{kbps}$ variations with an index of 0.5 and 0.75 from Figure 4.25 and Figure 4.27 originally and compared below in Figure 4.28. The AX5043's internal performance limitations decrease as modulation index increases. This seems logical, the receiver can differentiate the energy per bit in the spectrum better as deviation increases. In addition, Figure 4.29 below shows the evaluated modulation implementation losses of the compared indices. 
BER vs. $E_{b} / N_{0}$ for 60 kbps BFSK (closed systems)

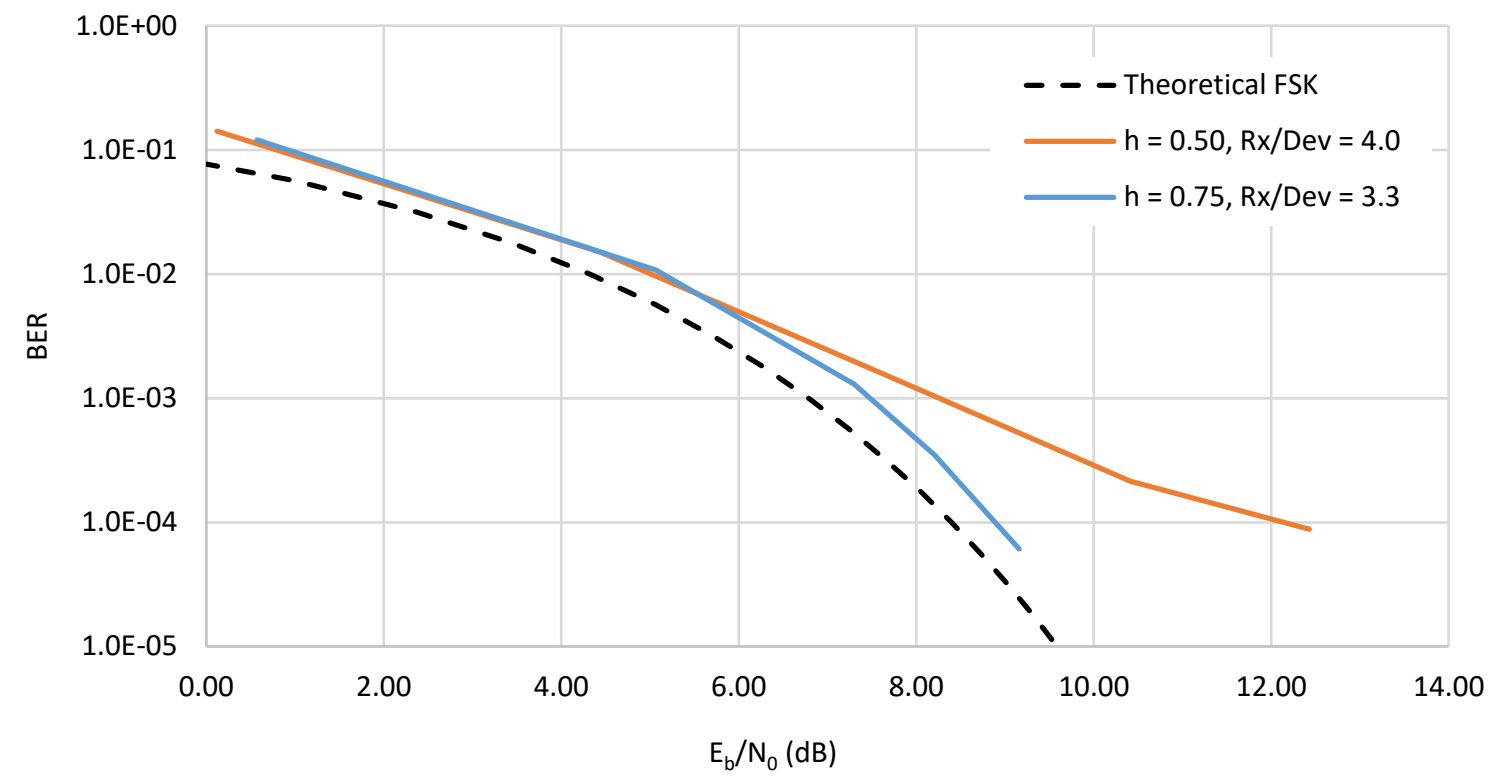

Figure 4.28: Modulation index influence on BER vs. $E_{b} / N_{o}$ plot for $60 \mathrm{kbps}$

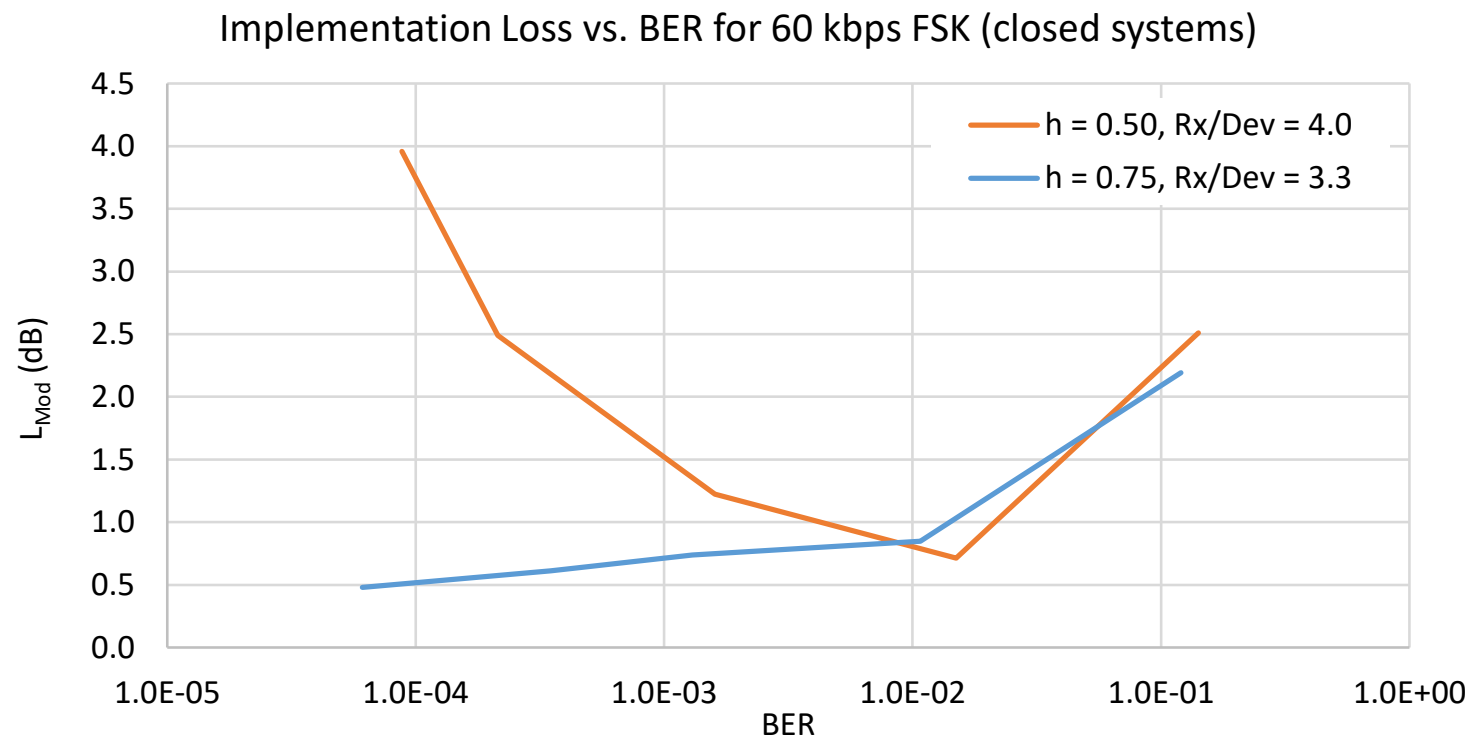

Figure 4.29: Modulation index influence on Implementation Loss vs. BER plot for $60 \mathrm{kbps}$

The performance limitations discovered for the AX5043 transceiver IC have allowed an ideal modulation scheme to be designed. A BFSK modulation scheme is chosen with an index $\left(h_{m}\right)$ of $0.667(2 / 3)$ instead of 0.5 due to being more resilient at 
lower BER values. A receiver bandwidth to carrier deviation ratio ( $\left.B_{R} / D e v\right)$ of 2 or greater is also ideal. Those parameters are used to determine the minimum receiver bandwidth to data rate ratio $\left(\Delta B_{R}\right)$, shown in Equations (4.36) and (4.37).

$$
\begin{gathered}
\frac{B_{R}}{D e v}=\frac{B_{R}}{R_{b} h_{m}} \geq 2 \\
\Delta B_{R}=\frac{B_{R}}{R_{b}} \geq 2 h_{m}=1.333
\end{gathered}
$$

A minimum receiver bandwidth to data rate ratio of 1.333 is ideal; a value of 1.5 is chosen. A value for the receiver's efficiency factor $\left(F_{R}\right)$ is calculated using Equation (4.38) which equates to $3.0 \mathrm{~dB}$.

$$
\begin{gathered}
F_{R}=10 \log _{10}\left[\Delta B_{R}\left(2-h_{m}\right)\right] \\
F_{R}=3.0 \mathrm{~dB} \text { for FSK, } h_{m}=0.667, \Delta B_{R}=1.5
\end{gathered}
$$

\subsection{Revised CubeSat Link Budget}

The communication uplink budget for the LEO CubeSat, OreSat1, can now be reanalyzed to include the revised $C N R$ to $E_{b} / N_{0}$ relationship, Equation (4.31), and the chosen receiver efficiency factor $\left(F_{R}\right)$ of $3.0 \mathrm{~dB}$. The minimum receiver power is first computed using the formula from section [2.7.1 Minimum Receiver Power] and including the $E_{b} / N_{0}$ revision, shown in Equation (4.39). Table 4.7 below, shows the spacecraft's (S/C) revised minimum receiver power for the three different data rates originally computed in section [2.7.1 Minimum Receiver Power]. All values are $3.0 \mathrm{~dB}$ higher than previously computed. 


$$
P_{R_{x}}=k+T_{S}+R_{b}+F_{R}+L_{M o d}+\frac{E_{b}}{N_{0_{R e q}}}
$$

\begin{tabular}{l|c|ccc|c} 
& & $\mathrm{R}_{\mathrm{b}}=$ & $\mathrm{R}_{\mathrm{b}}=$ & $\mathrm{R}_{\mathrm{b}}=$ & \\
Parameter & Specification & $10 \mathrm{kbps}$ & $60 \mathrm{kbps}$ & $120 \mathrm{kbps}$ & Units \\
\hline $\mathrm{k}$ & - & -198.6 & -198.6 & -198.6 & $\mathrm{dBm} / \mathrm{Hz}-\mathrm{K}$ \\
$\mathrm{R}_{\mathrm{b}}$ & see heading & 40.0 & 47.8 & 50.8 & $\mathrm{~dB}-\mathrm{Hz}$ \\
$\mathrm{T}_{\mathrm{S}}$ & $369 \mathrm{~K}$ & 25.7 & 25.7 & 25.7 & $\mathrm{~dB}-\mathrm{K}$ \\
$\mathrm{E}_{\mathrm{b}} / \mathrm{N}_{0}$ Req. & $10^{-4} \mathrm{BER}$ & 8.4 & 8.4 & 8.4 & $\mathrm{~dB}$ \\
$\mathrm{~F}_{\mathrm{R}}$ & $\Delta \mathrm{B}_{\mathrm{R}}=1.5, \mathrm{~h}_{\mathrm{m}}=2 / 3$ & 3.0 & 3.0 & 3.0 & $\mathrm{~dB}$ \\
$\mathrm{~L}_{\text {Mod }}$ & measured & 0.5 & 0.5 & 0.5 & $\mathrm{~dB}$ \\
\multicolumn{2}{l}{ Minimum Received Power: } & -121.0 & -113.2 & -110.2 & $\mathrm{dBm}$
\end{tabular}

Table 4.7: S/C Revised minimum receiver power

In addition, the Earth station's (ES) minimum transmitter power is reevaluated using the formula originally shown in [2.7.2 Minimum Transmitter Power] and shown again for convenience in Equation (4.40). Results are computed for the same three data rates and shown in Table 4.8.

$$
P_{T_{x}}=P_{R_{x}}+L_{\text {Point }}+L_{F S P L}+L_{A t m o s .}+L_{T_{x} . F e e d}-G_{T_{x} A n t .}-G_{R_{x} A n t .}
$$

\begin{tabular}{|c|c|c|c|c|c|}
\hline Parameter & Specification & $\begin{array}{c}R_{b}= \\
10 \mathrm{kbps}\end{array}$ & $\begin{array}{c}\mathrm{R}_{\mathrm{b}}= \\
60 \mathrm{kbps}\end{array}$ & $\begin{array}{c}R_{b}= \\
120 \mathrm{kbps}\end{array}$ & Units \\
\hline$P_{R x}$ & minimum & -121.0 & -113.2 & -110.2 & $\mathrm{dBm}$ \\
\hline $\mathrm{L}_{\mathrm{FSPL}}$ & $1,440 \mathrm{~km}$ & 157.7 & 157.7 & 157.7 & $d B$ \\
\hline L & estimated & 3.0 & 3.0 & 3.0 & $\mathrm{~dB}$ \\
\hline $\mathrm{L}_{\text {Tx.Feed }}$ & calculated & 1.8 & 1.8 & 1.8 & $d B$ \\
\hline LAtmos. & $10^{\circ}$ Elv. & 1.3 & 1.3 & 1.3 & $\mathrm{~dB}$ \\
\hline $\mathrm{G}_{\text {Rx.Ant }}$ & Turnstile & $(-) 1.4$ & $(-) 1.4$ & $(-) 1.4$ & $\mathrm{dBi}$ \\
\hline $\mathrm{G}_{\mathrm{TX} \text {.Ant }}$ & Quad Helix & $(-) 16.0$ & $(-) 16.0$ & $(-) 16.0$ & $\mathrm{dBi}$ \\
\hline \multirow{2}{*}{\multicolumn{2}{|c|}{ Minimum Transmit Power: }} & 25.4 & 33.2 & 36.2 & $\mathrm{dBm}$ \\
\hline & & 0.35 & 2.09 & 4.17 & W \\
\hline
\end{tabular}

Table 4.8: ES Revised minimum transmitter power 


\section{Conclusion}

An L-band communication system uplink budget was designed for a low Earth orbit (LEO) CubeSat and the receiver's threshold was validated using an anechoic chamber where a controlled thermal noise environment was established. An inexpensive receiver prototype was designed and constructed using COTS components. The receiver consumed only $130 \mathrm{~mW}$ with a noise figure measurement of $0.70 \mathrm{~dB}(50.9$ K) from the first LNA.

The receiver's theoretical expected carrier-to-noise ratio (CNR) for the bit-errorrate $(B E R)$ to energy per bit to noise power spectral density $\left(E_{b} / N_{0}\right)$ relationship was measured and found to not match the simple relationship to data rate. A revised relationship was conceived with dependencies on data rate, the receiver's bandwidth to data rate ratio, and the modulation index. The revised relationship proved to work well for a variety of binary frequency-shift keying (BFSK) modulation indices less than or equal to 0.75 with a minimum of 0.5 . In addition, a smaller implementation loss of 0.5 $\mathrm{db}$ at $10^{-4}$ BER was measured for an index of 0.75 .

Results from the communication system validation were used to provide improved link analysis for the CubeSat, OreSat1. The L-band uplink budget yielded a revised theoretical data rate operation of up to $120 \mathrm{kbps}$ with a $10^{\circ}$ spacecraft elevation and an Earth station transmitting only $4.2 \mathrm{~W}$ from its power amplifier using a $16 \mathrm{dBi}$ antenna for a link margin of zero. 


\subsection{Future Work}

The list below suggests future work for the L-band receiver discussed in this paper for the continuing development of the CubeSat, OreSat1, and further investigation of the AX5043 transceiver IC.

- Integration: The L-band receiver evaluation boards need to be integrated onto a single PCB.

- LNA Revision: The Infineon BFP740F LNA design should be revised to include the active biasing IC, Infineon BCR400W, to reduce the LNA's overall temperature coefficient.

- Validation: The receiver's proposed final BPSK modulation index of 0.667 for 10 , 60 , and $120 \mathrm{kbps}$ should be validated in a threshold measurement as discussed in Chapter 4.

- EMI: The integrated L-band receiver in the $\mathrm{S} / \mathrm{C}$ should be validated in an additional threshold measurement where it experiences electromagnetic interference (EMI) from any combination of other radios operating. 


\section{References}

[1] "Portland State Aerospace Society," 2020. [Online]. Available: http://psas.pdx.edu/.

[2] B. Klofas, J. Anderson and K. Leveque, "A Survey of CubeSat Communication Systems," November 2008. [Online]. Available: https://www.klofas.com/papers/CommSurvey-Bryan_Klofas.pdf.

[3] B. Klofas and K. Leveque, "A Survey of CubeSat Communication Systems: 20092012," April 2013. [Online]. Available: https://www.klofas.com/papers/Klofas_Communications_Survey_2009-2012.pdf.

[4] J. E. D. \&. P. J. Wertz, Space Mission Engineering : The New SMAD, Torrance, CA: Microcosm Press, 2011.

[5] W. L. Stutzman and G. A. Thiele, Antenna Theory and Design Third Edition, Hoboken, NJ: John Wiley \& Sons, Inc., 2013.

[6] J. D. Kraus, Radio Astronomy 2nd Edition, Durham, NJ: Cygnus-Quasar Books, 1986.

[7] M. F. Mesiya, Contemporary Communication Systems, New York, NY: The McGraw-Hill Companies, Inc., 2013.

[8] J. King, "The Jan King Link Budget Spread Sheets," AMSAT-UK, [Online]. Available: http://www.amsatuk.me.uk/iaru/spreadsheet.htm.

[9] W. Hayward, R. Campbell and B. Larkin, Experimental Methods in RF Desgin, Newington, CT: American Radio Relay League, 2009.

[10] Keysight Technologies, "Noise Figure Measurement Accuracy: The Y-Factor Method," March 2019. [Online]. Available: https://www.keysight.com/us/en/assets/7018-06829/application-notes/59523706.pdf.

[11] C. Bowick, J. Blyler and C. Ajluni, RF Circuit Design Second Edition, Burlington, MA: Newnes/Elsevier Inc., 2008.

[12] G. Gonzalez, Microwave Transistor Amplifiers Second Edition, Upper Saddle River, NJ: Prentice-Hall, Inc., 1997.

[13] ON Semiconductor, "AX5043: Ultra-Low Power Narrow-Band Sub GHz (27 - 1050 $\mathrm{MHz}$ ) RF Transceiver," October 2015. [Online]. Available: https://www.onsemi.com/pub/Collateral/AX5043-D.PDF.

[14] ON Semiconductor, "DVK-BASE-2-GEVK: DVK-2 Base Evaluation Kit," ON Semiconductor, 2020. [Online]. Available: https://www.onsemi.com/support/evaluation-board/dvk-base-2-gevk.

[15] ON Semiconductor, "AND9356/D: AX-RadioLAB for AX5043 User Manual," June 2016. [Online]. Available: https://www.onsemi.com/pub/Collateral/AND9356D.PDF. 
[16] Silicon Labs`, "Si4133-D-GT RF Synthesizers," Silicon Labs, 2020. [Online]. Available: https://www.silabs.com/wireless/proprietary/rf-synthesizers/device.si4133-d-gt.

[17] Maxim Integrated, "HFTA-10.0: PHYSICAL LAYER PERFORMANCE: TESTING THE BIT ERROR RATIO (BER)," 1 December 2004. [Online]. Available: https://www.maximintegrated.com/en/design/technical-documents/appnotes/3/3419.html.

[18] Z.-D. W and S.-Y. QIN, "Effect Study of Spectrum Analyzer Noise Floor on Antenna Noise Temperature Measurement," in IEEE; Antennas, Propagation \& EM Theory (ISAPE), 2012 10th International Symposium on, Piscataway, NJ, 2012.

[19] J. Miller, "9600 Baud Packet Radio Modem Design by James Miller G3RUH," 31 October 2005. [Online]. Available: https://www.amsat.org/amsat/articles/g3ruh/109.html. 